\title{
Hot carrier dynamics and electron-optical phonon coupling in photoexcited graphene via time-resolved ultrabroadband terahertz spectroscopy
}

\author{
Sho Ikeda, Chiko Otani, and Masatsugu Yamashita $\odot^{*}$ \\ Terahertz Sensing and Imaging Team, RIKEN Center for Advanced Photonics, \\ 519-1399 Aramaki-Aoba Aoba-ku, Sendai, Miyagi, 980-0845, Japan
}

(Received 5 July 2021; revised 15 September 2021; accepted 21 October 2021; published 29 November 2021)

\begin{abstract}
Electron-electron (e-e) interaction is known as a source of logarithmic renormalizations for Dirac fermions in quantum field theory. The renormalization of electron-optical phonon coupling (EPC) by e-e interaction, which plays a pivotal role in hot carrier and phonon dynamics, has been discussed since the discovery of graphene. We investigate hot carrier dynamics and EPC strength using time-resolved ultrabroadband terahertz (THz) spectroscopy combined with numerical simulation based on the Boltzmann transport equation and a comprehensive temperature model. The numerical simulation demonstrates that the extrinsic carrier scatterings by the Coulomb potential of the charged impurity and surface polar phonons are significantly suppressed by the carrier screening effect and have negligible contributions to the $\mathrm{THz}$ photoconductivity in heavily doped graphene on polyethylene terephthalate (PET) substrate. The large negative photoconductivity and the non-Drude behavior of $\mathrm{THz}$ conductivity spectra appear under high pump fluence and can be attributed to the temporal variation of the hot carrier distribution and scattering rate. The transient reflectivity well reflects the EPC strength and temporal evolution of the hot carrier and optical phonon dynamics. We successfully estimate the EPC matrix element of the $A_{1}^{\prime}$ optical phonon mode near the $\mathbf{K}$ point as $\left\langle D_{\mathbf{K}}^{2}\right\rangle_{\mathrm{F}} \approx 450(\mathrm{eV} / \AA)^{2}$ from the fitting of $\mathrm{THz}$ conductivity spectra and temporal evolution of transient $\mathrm{THz}$ reflectivity. The corresponding dimensionless EPC constant $\lambda_{\mathbf{K}} \approx 0.09$ at Fermi energy $\varepsilon_{\mathrm{F}}=0.43 \mathrm{eV}$ is slightly larger than the prediction of the renormalization group approach including the dielectric screening effect of the PET substrate. This leads to a significant difference in hot carrier and phonon dynamics compared with those without the renormalization effect by the e-e interaction. This approach can provide a quantitative understanding of hot carrier and optical phonon dynamics and support the development of future graphene optoelectronic devices.
\end{abstract}

DOI: 10.1103/PhysRevResearch.3.043143

\section{INTRODUCTION}

Hot carrier effects are regarded as insightful in studying many-body interactions in condensed matter and play a crucial role in the operation of electronics and optoelectronic devices. For this reason, they have been investigated extensively in both metals and semiconductors [1,2]. The rise of graphene offered new opportunities for this research field because the carriers thereof are two-dimensional (2D) massless Dirac fermions (MDFs) with a linear energy dispersion. This fact has promoted graphene as an attractive platform for hot carrier physics and various applications [3-23]. Electron or hole relaxation mainly involves nonradiative electron-electron (e-e) and electron-phonon scatterings, depending on the excitation energy. Electron-electron interaction is dominant at high energy, redistributes the electrical or optical power within

\footnotetext{
*m-yama@ riken.jp

Published by the American Physical Society under the terms of the Creative Commons Attribution 4.0 International license. Further distribution of this work must maintain attribution to the author(s) and the published article's title, journal citation, and DOI.
}

the electron gas, and builds up a hot carrier population. Electron-phonon interaction operates on a longer time scale to equilibrate the electron and phonon temperatures and to cool the hot carriers [24].

Hot carrier effects play a significant role in the optoelectronic properties of photoexcited graphene, in which the photocarriers are excited at high energies. The subsequent relaxation drives the working efficiency of optoelectronic devices. In this respect, spectroscopic investigations such as pump probe spectroscopy [25] and angle-resolved photoemission spectroscopy [26,27] of hot carriers complement transport studies. Optical pump terahertz $(\mathrm{THz})$ probe spectroscopy (OPTP) is a powerful tool for investigating the hot carrier dynamics of graphene because it probes the intraband optical conductivity dominated not only by the hot carrier distribution but also by the carrier-scattering process in contrast to optical pump optical probe spectroscopy. Extensive studies using OPTP [28-41] have revealed the unusual behaviors of graphene hot carriers, which undergo positive and negative changes in the intraband optical conductivity with non-Drudetype frequency dependence. The negative change observed in heavily doped graphene is indicative of enhanced carrier scattering and reduced Drude weight in a quasiequilibrium hot carrier state with a single chemical potential owing to ultrafast 
recombination of photoexcited carriers. However, most of these works were performed by $\mathrm{THz}$ probe with a relatively narrow band $(1-3 \mathrm{THz})$, which was not sufficient for capturing the whole spectrum of non-Drude-type conductivity, and their results have been interpreted using the framework of the phenomenological model $[31,33,36,37,40]$. Such a phenomenological analysis for the narrowband spectra is not sufficient to understand the hot carrier and phonon dynamics quantitatively and to derive the microscopic parameters. Theoretical studies have been conducted by incorporating the microscopic theory based on the density matrix formalism [41] or Boltzmann transport equation (BTE) [42,43].

The electron-optical phonon coupling (EPC) strength is a crucial factor that makes it difficult to understand the hot carrier and phonon relaxation process by numerical studies. Density functional theory (DFT) calculations demonstrated that only three strongly coupled optical phonon (SCOP) modes contribute significantly to the inelastic carrier scattering in graphene [44,45]. The first two relevant modes are associated with the $G$ peak of the Raman spectrum and the highest optical branches at $\boldsymbol{\Gamma}$ (the $E_{2 g}$ mode) with an energy of $\hbar \omega_{\Gamma}=196.0 \mathrm{meV}$, which split into an upper longitudinal optical (LO) branch and a lower transverse optical (TO) branch near $\boldsymbol{\Gamma}$. Owing to their long wavelengths, these phonons scatter electrons within one valley. Moreover, it is essential to take into account the highest optical branch of the zone boundary phonon $\hbar \omega_{\mathbf{K}}=161 \mathrm{meV}$ at the $\mathbf{K}$ point (the $A_{1}^{\prime}$ mode). This mode is responsible for intervalley processes and associated with the $\mathrm{D}$ and 2D peaks of the Raman spectrum. In Refs. [44,46-48], the EPCs $\left\langle D_{\eta}^{2}\right\rangle_{\mathrm{F}}$ for dominant optical phonon modes $\eta\left(\boldsymbol{\Gamma}_{\mathrm{LO}}, \boldsymbol{\Gamma}_{\mathrm{TO}}, \mathbf{K}\right)$ were defined as the average on the Fermi surface of the matrix element $\left|D_{\lambda k \lambda k^{\prime}}^{\eta}\right|$ of the Kohn-Sham potential, differentiated with respect to the phonon displacement. The EPC for LO and TO modes at the $\Gamma$ point had $\left\langle D_{\Gamma}^{2}\right\rangle_{\mathrm{F}}=45.6(\mathrm{eV} / \AA)^{2}$, which was in good agreement with experimental results [49]. However, the EPC value at the $\mathbf{K}$ point has been debated [47,49-52] because it is renormalized by the e-e interaction and is affected by the presence of the substrate owing to the dielectric screening effect [53]. The amount calculated by DFT with the generalized gradient approximation was $\left\langle D_{\mathbf{K}}^{2}\right\rangle_{\mathrm{F}}=92.0(\mathrm{eV} / \AA)^{2}$ [44]. However, a Hedin's $G W$ approximation (GW) calculation, which considers the e-e interaction by approximating the self-energy using the product of the Green's function and screened Coulomb potential, but neglects the vertex corrections, yielded $\left\langle D_{\mathbf{K}}^{2}\right\rangle_{\mathrm{F}}=193(\mathrm{eV} / \AA)^{2}[47,54,55]$.

In this paper, we investigate the hot carrier dynamics in photoexcited heavily doped graphene on a polyethylene terephthalate (PET) substrate using an OPTP and estimate the EPC strength at the $\mathbf{K}$ point via a numerical simulation based on the combination of BTE and a comprehensive temperature model [43]. Owing to the small change in the Drude weight of heavily doped graphene and the negligible contribution of charged impurities and surface optical phonons (SOPs) of the PET substrate, the rise and relaxation dynamics of the SCOP are effectively captured by the transient $\mathrm{THz}$ reflectivity change measured by ultrabroadband $\mathrm{THz}$ probe. Using the renormalization group analysis, the obtained dimensionless EPC at the $\mathbf{K}$ point is discussed and compared with those determined by other techniques.

\section{SIMULATION METHOD AND RESULTS}

In this section, we present a numerical simulation of the $\mathrm{THz}$ conductivity and the transient $\mathrm{THz}$ reflectivity measured by the OPTP experiment according to the following procedures. After photoexcitation, photoexcited carriers are quickly recombined, and their energy is redistributed within the electron gas forming a hot carrier state in quasiequilibrium with a single chemical potential. A number of cooling pathways for hot carriers by inelastic scattering have been proposed such as SCOPs [41,56,57], acoustic phonons [58-61], and SOPs of the substrate [62]. As we will explain later, the contribution of the SOP and its coupled mode with plasmons can be neglected by selecting the substrate with low polarizability and small phonon energy $\hbar \omega_{\text {so }}$ [63-65]. The effect of acoustic phonons on hot carrier cooling is considered by the supercollision process, and the acoustic phonon occupation is assumed to remain unchanged from the equilibrium state in the picosecond time scale after photoexcitation [27]. Therefore we use a comprehensive temperature model to calculate the temporal evolution of the temperature for hot carriers in quasiequilibrium and the occupations for three dominant SCOP modes. Thereafter, the iterative solution of the BTE [43] is used to calculate the intraband complex conductivity of the hot carriers in the $\mathrm{THz}$ region. Because interband transition is forbidden at a THz probe energy of $\hbar \omega_{\mathrm{THz}}<2 \varepsilon_{\mathrm{F}}$, the THz conductivity of doped graphene is dominated by the intraband transition. This scheme enables us to reduce the computational cost substantially compared with the calculation of the full solutions of the coupled graphene Bloch equation and BTEs for hot carriers and hot phonon modes in 2D momentum space.

\section{A. THz conductivity calculation}

The iterative solution of the BTE for obtaining the steadystate and dynamical conductivity of semiconductors was introduced in Refs. [66,67] and was subsequently modified for 2D MDFs in graphene [43]. The temporal evolution of the carrier distribution is described by the BTE under a timedependent electric field, which is expressed as

$$
\frac{\partial f_{\lambda}(\boldsymbol{k}, t)}{\partial t}=-\frac{(-e)}{\hbar} \boldsymbol{E}(t) \frac{\partial f_{\lambda}(\boldsymbol{k}, t)}{\partial \boldsymbol{k}}+\left.\frac{\partial f_{\lambda}(\boldsymbol{k}, t)}{\partial t}\right|_{\mathrm{c}} .
$$

Here, $f_{\lambda}(\boldsymbol{k}, t)$ is the electron distribution function for the conduction band $(\lambda=1)$ and valence band $(\lambda=-1), k$ is the wave vector of the carriers, $e$ is the elementary charge, and $\boldsymbol{E}(t)$ is the electric field of the THz probe pulse. $\partial f_{\lambda}(\boldsymbol{k}, t) /\left.\partial t\right|_{\mathrm{c}}$ is the collision term that describes the change in the distribution function via carrier scattering.

We consider the intrinsic carrier-scattering mechanism by the optical and acoustic phonon modes [68-77] and the extrinsic mechanism by the charged impurities $[68,69,78-80]$ and weak scatterers $[70,71,81-86]$. For spherical bands under a low field $\boldsymbol{E}$, the general solution of Eq. (1) is approximately provided by the first two terms of the zone spherical expansion.

$$
f_{\lambda}(\boldsymbol{k}, t)=f_{0}\left(\varepsilon_{\lambda \boldsymbol{k}}\right)+g\left(\varepsilon_{\lambda \boldsymbol{k}}, t\right) \cos \alpha_{\boldsymbol{k}},
$$

where $f_{0}\left(\varepsilon_{\lambda k}\right)=1 /\left[\exp \left\{\left[\varepsilon_{\lambda k}-\mu\left(T_{e}\right)\right] / k_{\mathrm{B}} T_{e}\right\}+1\right]$ is the Fermi-Dirac distribution for the corresponding equilibrium 
electron distribution at the electron temperature $T_{e}$. $\varepsilon_{\lambda \boldsymbol{k}}= \pm \hbar v_{\mathrm{F}}|\boldsymbol{k}|\left(\varepsilon_{1 \boldsymbol{k}} \geqslant 0\right.$ and $\varepsilon_{-1 \boldsymbol{k}} \leqslant 0$ for the conduction and valence bands, respectively) is the electron energy within the Dirac approximation of the graphene energy-band structure [87], and $v_{\mathrm{F}}$ is the Fermi velocity. In this expression, $\mu\left(T_{e}\right)$ is the temperature-dependent chemical potential of the 2D MDFs [36,79,88] [illustrated in Fig. 3(a)]. $g\left(\varepsilon_{\lambda k}, t\right)$ is the perturbation part of the distribution, and $\alpha_{k}$ is the angle between $\boldsymbol{E}$ and $\boldsymbol{k}$.

In Eq. (1), the collision term is given by

$$
\left.\frac{\partial f_{\lambda}(\boldsymbol{k}, t)}{\partial t}\right|_{\mathrm{c}}=\sum_{\eta, \lambda^{\prime}} C_{\lambda \lambda^{\prime}}^{\eta}(\boldsymbol{k})+C_{\lambda}^{\mathrm{el}}(\boldsymbol{k}),
$$

while accounting for the scattering of the electrons with dominant optical phonon modes $\eta$, in $C_{\lambda \lambda}^{\eta}$, including both the intraband $\left(\lambda=\lambda^{\prime}\right)$ and interband $\left(\lambda \neq \lambda^{\prime}\right)$ processes with elastic scattering processes in $C_{\lambda}^{\mathrm{el}}(\boldsymbol{k})$. The carrier collision term $C_{\lambda \lambda^{\prime}}^{\eta}(\boldsymbol{k})$ for the interaction of the electron and optical phonons is expressed as

$$
\begin{aligned}
C_{\lambda \lambda^{\prime}}^{\eta}(\boldsymbol{k})= & \sum_{\mathbf{k}^{\prime}}\left\{P_{\lambda^{\prime} \mathbf{k}^{\prime} \lambda \mathbf{k}}^{\eta} f_{\lambda^{\prime}}\left(\mathbf{k}^{\prime}\right)\left[1-f_{\lambda}(\mathbf{k})\right]\right. \\
& \left.-P_{\lambda \mathbf{k} \lambda^{\prime} \mathbf{k}^{\prime}}^{\eta} f_{\lambda}(\mathbf{k})\left[1-f_{\lambda^{\prime}}\left(\mathbf{k}^{\prime}\right)\right]\right\},
\end{aligned}
$$

where $P_{\lambda^{\prime} \mathbf{k}^{\prime} \lambda \mathbf{k}}^{\eta}$ and $P_{\lambda \mathbf{k} \lambda^{\prime} \mathbf{k}^{\prime}}^{\eta}$ are the transition rate by the optical phonon modes, $\eta$, between states $\left(\mathbf{k}^{\prime}, \lambda^{\prime}\right) \rightarrow(\mathbf{k}, \lambda)$ and $(\mathbf{k}, \lambda) \rightarrow\left(\mathbf{k}^{\prime}, \lambda^{\prime}\right)$, respectively. $P_{\mathbf{k} \lambda \mathbf{k}^{\prime} \lambda^{\prime}}^{\eta}$ is expressed by

$$
P_{\lambda \boldsymbol{k} \lambda^{\prime} \boldsymbol{k}^{\prime}}^{\eta}=P_{\lambda \boldsymbol{k} \lambda^{\prime} \boldsymbol{k}^{\prime}}^{\mathrm{EM}, \eta}+P_{\lambda \boldsymbol{k} \lambda^{\prime} \boldsymbol{k}^{\prime}}^{\mathrm{AB}, \eta}
$$

which accounts for the phonon emission and absorption, given by

$$
\begin{aligned}
P_{\lambda \boldsymbol{k} \lambda^{\prime} \boldsymbol{k}^{\prime}}^{\mathrm{EM} / \mathrm{AB}, \eta}= & \frac{\pi\left|D_{\lambda \boldsymbol{k} \lambda^{\prime} \boldsymbol{k}^{\prime}}^{\eta}\right|^{2}}{\rho \omega_{\eta} A}\left(n_{\eta}+\frac{1}{2} \pm \frac{1}{2}\right) \\
& \times \delta\left(\varepsilon_{\lambda \boldsymbol{k}}-\varepsilon_{\lambda^{\prime} \boldsymbol{k}^{\prime}} \mp \hbar \omega_{\eta}\right) \delta\left(\boldsymbol{k}-\boldsymbol{k}^{\prime} \mp \boldsymbol{q}\right) .
\end{aligned}
$$

Here, $\left|D_{\lambda k \lambda / k^{\prime}}^{\eta}\right|$ is the EPC matrix element defined in Ref. [44], $\boldsymbol{k}^{\prime}=\boldsymbol{k} \pm \boldsymbol{q}$, and $\boldsymbol{q}$ is the wave vector of the optical phonons. $\rho=7.6 \times 10^{-7} \mathrm{~kg} / \mathrm{m}^{2}$ is the area density of graphene, and $A$ is the area of the graphene sample, whereas $\omega_{\eta}$ and $n_{\eta}$ are the angular frequency and occupation of the optical phonons, respectively.

The carrier-scattering rates that are obtained by the optical phonons in Eq. (6) account for the phonon emission and absorption. For small $\boldsymbol{q}$ and $\boldsymbol{k}^{\prime}$, the EPC matrix elements $\left|D_{\lambda k \lambda^{\prime} \boldsymbol{k}^{\prime}}^{\eta}\right|^{2}$ for the $\boldsymbol{\Gamma}_{\mathrm{LO}}, \boldsymbol{\Gamma}_{\mathrm{TO}}$, and $\mathbf{K}$ phonons are expressed by $[44,89]$

$$
\begin{aligned}
\left|D_{\lambda \boldsymbol{k} \lambda^{\prime} \boldsymbol{k}^{\prime}}^{\boldsymbol{\Gamma}_{\mathrm{LO} / \mathrm{TO}}}\right|^{2} & =\left\langle D_{\boldsymbol{\Gamma}}^{2}\right\rangle_{\mathrm{F}}\left\{1 \pm \cos \left(\theta_{\boldsymbol{k}, \boldsymbol{q}}+\theta_{\boldsymbol{k}^{\prime}, \boldsymbol{q}}\right)\right\}, \\
\mid D_{\lambda \boldsymbol{k} \lambda^{\prime} \boldsymbol{k}^{\prime}}^{\mathbf{K}} & \left.\right|^{2}=\left\langle D_{\mathbf{K}}^{2}\right\rangle_{\mathrm{F}}\left\{1 \pm \cos \theta_{\boldsymbol{k}, \boldsymbol{k}^{\prime}}\right\} .
\end{aligned}
$$

Here, $\theta_{\boldsymbol{k}, \boldsymbol{q}}$ denotes the angle between $\boldsymbol{k}$ and $\boldsymbol{q}, \theta_{\boldsymbol{k}^{\prime}, \boldsymbol{q}}$ denotes the angle between $\boldsymbol{k}^{\prime}$ and $\boldsymbol{q}$, and $\theta_{\boldsymbol{k}, \boldsymbol{k}^{\prime}}$ denotes the angle between $\boldsymbol{k}$ and $\boldsymbol{k}^{\prime}$. In the case of $\boldsymbol{\Gamma}_{\mathrm{LO}}$ and $\mathbf{K}$ phonons, the plus sign refers to the interband processes, and for $\boldsymbol{\Gamma}_{\mathrm{TO}}$ phonons, it refers to the intraband processes.

In Eq. (3), the elastic term $C_{\lambda}^{\mathrm{el}}(\boldsymbol{k})$ is calculated using the elastic scattering rate $P_{\lambda k \lambda k^{\prime}}^{s}$ [43]. The index, $s$, refers to the different elastic scattering modes by weak scatterers, and charged impurities, which are characterized by resistivity of the weak scatterers $\rho_{\mathrm{s}}$, and charged impurity concentration $n_{\mathrm{i}}$, respectively. The reported $\rho_{\mathrm{s}}$ ranges from 40 to $100 \Omega[70,71,85,86]$. Interactions with acoustic phonons are treated as quasielastic and included in $C_{\lambda}^{\mathrm{el}}(\boldsymbol{k})$. Different electron-acoustic phonon coupling models have been proposed to extract the effective coupling constant $J_{\mathrm{a}}$ from experimental data for graphene, which ranges from 10 to $30 \mathrm{eV}$ [71,72,74-76,82,90-99]. A first-principles study suggests that the gauge-field contribution is more important than the screened deformation potential $[100,101]$.

The iterative solution of $g^{j}\left(\varepsilon_{\lambda k}\right)=g\left(\varepsilon_{\lambda k}, t_{j}\right)$ is provided by

$$
g^{j+1}\left(\varepsilon_{\lambda k}\right)=\frac{S_{\lambda}^{\text {in }}-\frac{(-e) E^{j}}{\hbar} \frac{\partial f_{0}}{\partial k}+\Omega_{\mathrm{s}} g^{j}}{S_{\lambda}^{\text {out }}+v^{\text {el }}+\Omega_{\mathrm{s}}} .
$$

Here, $E^{j}=\left|\boldsymbol{E}\left(t_{j}\right)\right|$ and $k=|\boldsymbol{k}|$ are the magnitudes of the electric field and wave vector, respectively. $\Omega_{\mathrm{s}}$ is known as the self-scattering rate, and $1 / \Omega_{\mathrm{s}}$ is the time increment between successive iterations; $S_{\lambda}^{\text {in }}$ and $S_{\lambda}^{\text {out }}$ are the net inand out-scattering rates for inelastic scattering, respectively. Furthermore, $v^{\text {el }}$ is the total relaxation rate by the elastic scattering mechanisms. The sequence $\left\{g^{j}\left(\varepsilon_{\lambda \boldsymbol{k}}\right)\right\}$ yields $f_{\lambda}\left(\boldsymbol{k}, t_{j}\right)$ versus time when $\Omega_{\mathrm{s}}$ is sufficiently large compared with $S_{\lambda}^{\text {out }}+v^{\mathrm{e}}$.

\section{B. Temperature model of hot carriers}

The hot carrier intraband optical conductivity $\sigma\left(\omega, \tau_{1}\right)$ in the cooling process can be calculated from $f_{\lambda}\left(\boldsymbol{k}, t_{j}\right)$, which is obtained by substituting the hot carrier and three dominant optical phonon temperatures $\left[T_{e}\left(t_{j}\right), T_{\eta}\left(t_{j}\right)\right]$ into Eq. (8) in the iteration process. Here, $\tau_{1}$ is the pump probe delay. We employ coupled rate equations for a comprehensive temperature model that describes the temperature evolutions of the electron temperature $T_{e}$ and optical phonon occupations $n_{\eta}$ by photoexcitation:

$$
\begin{aligned}
\frac{d T_{e}}{d t} & =\frac{\mathcal{I}_{\mathrm{ab}}-\sum_{\eta} R_{\eta}^{\mathrm{Net}} \hbar \omega_{\eta}-J_{\mathrm{sc}}}{C}, \\
\frac{d n_{\eta}}{d t} & =R_{\mathrm{M}, \eta}^{\mathrm{Net}}-\frac{n_{\eta}-n_{\eta 0}}{\tau_{\mathrm{ph}}} .
\end{aligned}
$$

In this case, $\mathcal{I}_{\mathrm{ab}}$ represents the pump intensity absorbed in the graphene sample during laser irradiation, considering the multiple reflections inside the substrate with dielectric constant $\epsilon\left(\omega_{\text {pump }}\right)=2.4$ for the pump wavelength and saturable absorption (SA) effect in graphene. $C$ is the sum of the specific heat of the electrons in the conduction and valence bands, $R_{\eta}^{\mathrm{Net}}=R_{\eta}-G_{\eta}$ denotes the total balance between the optical phonon emission and absorption rate, and $J_{\text {sc }}$ indicates the energy loss rate for the supercollision (SC) carrier-cooling process $[59,102] . R_{M, \eta}^{\mathrm{Net}}=R_{\mathrm{M}, \eta}-G_{\mathrm{M}, \eta}$ denotes the total balance between the optical phonon emission and absorption rate per number of phonon modes that participate in the carrier scattering. In calculations of $R_{\eta}^{\mathrm{Net}}$ and $R_{M, \eta}^{\mathrm{Net}}$, we include the scattering angle dependence of the $\left|D_{\lambda \boldsymbol{k}, \lambda^{\prime} \boldsymbol{k}^{\prime}}^{\eta}\right|^{2}$ in Eq. (7), which was not been considered in the temperature model used in the previous study [43,102-104]. Moreover, $n_{\eta 0}$ represents the phonon occupation near the $\boldsymbol{\Gamma}$ and $\mathbf{K}$ points, respectively, in equilibrium at room temperature, whereas $\tau_{\mathrm{ph}}$ is the 
phenomenological optical phonon decay time to other phonon modes via the phonon-phonon interaction caused by lattice anharmonicity [105]. The effective optical phonon temperatures are calculated by inverting the Bose-Einstein distribution function, $n_{\eta}=1 /\left(e^{\hbar \omega_{\eta} / k_{\mathrm{B}} T_{\eta}}-1\right)$. The formula and temperature dependence of $C, R_{\eta}^{\mathrm{Net}}, J_{\mathrm{sc}}$, and $R_{M, \eta}^{\mathrm{Net}}$ can be found in Ref. [43] and Appendix C.

The optical pump pulse is absorbed by the interband transition, and the absorption coefficient for freestanding graphene at the normal incidence is $\alpha_{\text {inter }}=\pi \alpha=0.23 \%$ under sufficiently weak pump conditions, where $\alpha$ is the fine structure constant. However, the SA effect in graphene under the intense pump fluence [108-111] should be considered. The $\mathrm{SA}$ is a nonperturbative, nonlinear optical phenomenon that depends on the pump power as well as the temperature and Fermi energy. Based on the theory given by Marini et al. [111], we derived the formula of $\mathcal{I}_{\mathrm{ab}}$ considering the SA and multiple reflections inside the substrate at the oblique angle of incidence for the temperature calculation in the experimental condition (see Appendix D):

$$
\mathcal{I}_{a b}(t)=\mathcal{I}_{0}(t) A_{12}^{s *}+\sum \mathcal{I}_{n}(t+n \Delta T) A_{21}^{s *},
$$

where $\mathcal{I}_{0}(t)$ is the envelope function of the incident pump pulse, which is assumed to have hyperbolic secant form, $\mathcal{I}_{0}(t)=\left(F_{0} / 2 \tau_{\text {pump }}\right) \operatorname{sech}^{2}\left(t / \tau_{\text {pump }}\right)$. In this case, $F_{0}$ is the incident fluence, and $2 \tau_{\text {pump }}$ is the pump pulse duration. $\mathcal{I}_{n}(t+$ $n \Delta T)=\left(F_{n} / 2 \tau_{\text {pump }}\right) \operatorname{sech}^{2}\left[(t+n \Delta T) / \tau_{\text {pump }}\right]$ represents the pump pulse by the $n$th multiple reflection of the incident pump pulse inside the substrate, where $F_{\mathrm{n}}$ is the fluence and $n \Delta T$ is the round-trip time for the $n$th reflection pump pulse in the substrate. $A_{\mathrm{ij}}^{s *}\left(F_{0} / 2 \tau_{\text {pump }}\right)$ is the absorption coefficient including the carrier temperature dependence of the SA effect at the interface of layer $i$ /graphene/layer $j$ when the pump pulse excites the graphene from layer $i$ (see Fig. 10 of Appendix A). In this model, the SA is characterized by the inelastic carrier relaxation time $\tau_{\text {ie }}$. [The pump intensity dependence of the interband absorption coefficient $\alpha_{\text {inter }}$ for the freestanding graphene and $A_{\mathrm{ij}}^{s *}\left(F_{0} / 2 \tau_{\text {pump }}\right)$ for the graphene on the substrate can be seen in Figs. 11 and 12 of Appendix D.]

\section{Simulation for graphene on PET substrate}

In the simulation, the carrier scattering by SOPs of the substrate is not included, whereas the SOPs play crucial roles for the carrier dynamics in graphene on a polar substrate [91,112-116]. The square of the EPC matrix element between a SOP and a carrier is proportional to

$$
g_{\mathrm{SO}} \frac{e^{-q d}}{q+q_{s}} .
$$

Here, $g_{\mathrm{SO}}=\beta e^{2} \hbar \omega_{\mathrm{SO}} / 2 \epsilon_{0}, \omega_{\mathrm{SO}}$ is the angular frequency of the SOP, $\varepsilon_{0}$ is the permittivity of the vacuum, and $d$ is the equilibrium distance of the graphene sheet from the substrate surface. $q$ is the angular wave number of the surface phonon, $q_{s}$ is the Thomas-Fermi screening constant of the 2D carriers, and

$$
\beta=\frac{\epsilon_{\mathrm{s}}-\epsilon_{\infty}}{\left(\epsilon_{\mathrm{s}}+1\right)\left(\epsilon_{\infty}+1\right)},
$$

where $\epsilon_{\mathrm{s}}$ and $\epsilon_{\infty}$ are the low- and high-frequency dielectric constant, respectively. $\beta$ is a measure of the polarizability of the dielectric interface.

For example, in crystalline $\mathrm{SiO}_{2},\left(\epsilon_{\mathrm{s}}=3.9, \epsilon_{\infty}=2.5\right)$ has two SOP modes at $\hbar \omega_{\mathrm{s} 1}=60.0 \mathrm{meV}$ and $\hbar \omega_{\mathrm{s} 2}=146.5 \mathrm{meV}$, with $\beta_{1}=0.025$ and $\beta_{2}=0.062$, respectively. These values correspond to $g_{\mathrm{SO} 1}=0.14 \mathrm{eV}^{2} / \AA$ and $g_{\mathrm{SO} 2}=0.82 \mathrm{eV}^{2} / \AA$ and are enhanced by roughly $50 \%$ in conventional $\mathrm{SiO}_{2}$ glass with $\epsilon_{\infty}=2.1$. As a result, the temperature dependence of carrier transport is dominated by SOP scattering in graphene on polar substrate such as $\mathrm{SiO}_{2}$ and $\mathrm{HfO}_{2}$ [91,114]. The energy loss rate of the hot carrier by SOP modes is given as $R_{\mathrm{SO}}^{\mathrm{Net}} \propto g_{\mathrm{SO}} \hbar \omega_{\mathrm{SO}}$ so that the large $\hbar \omega_{\mathrm{SO}}$ also affects the hot carrier dynamics significantly. The dispersion relation of SOP modes can be altered by the coupling of the plasmon and SOP in doped graphene. These effects change significantly the hot carrier dynamics and make the simulation more complex leading to hindering the estimation of EPC at the $\mathbf{K}$ point.

Therefore, in this paper, we select a graphene sample on a PET substrate which has the low polarizability $\left(\epsilon_{\mathrm{s}}=3.0\right.$, $\left.\epsilon_{\infty}=2.54\right)$ owing to the polar low-frequency vibrational modes around $10 \mathrm{meV}$ [106]. The $g_{\mathrm{SO}}=0.029 \mathrm{eV}^{2} / \AA$ of PET is small and decreases significantly in doped graphene by the carrier screening effect. The $R_{\mathrm{SO}}^{\mathrm{Net}}$ between carriers and SOP of PET is expected to be smaller by three orders of magnitude than $\mathrm{SiO}_{2}$ and makes a negligible contribution to hot carrier cooling and $\mathrm{THz}$ conductivity. Furthermore, the small static dielectric constant $\epsilon_{\mathrm{s}}=3.0$ of a PET substrate provides weak dielectric screening with an expected larger renormalization effect on the EPC by e-e interaction [53].

The transient reflection change $\Delta E_{\mathrm{r}}\left(\tau_{1}\right) / E_{0}$ of graphene on PET substrate with the dielectric constant $\epsilon\left(\omega_{\mathrm{THz}}\right)=2.5$ in $\mathrm{THz}$ region can be calculated from $\sigma\left(\omega, \tau_{1}\right)$. (For details, see Appendix E.) In this case, $\Delta E_{\mathrm{r}}\left(\tau_{1}\right) / E_{0}$ is defined as $\Delta E_{\mathrm{r}}\left(\tau_{1}\right) / E_{0} \equiv\left[E_{\mathrm{r}}\left(\tau_{2}, \tau_{1}\right)-E_{\mathrm{r}}\left(\tau_{2}\right)\right] / E_{\mathrm{r}}\left(\tau_{2}\right)$ at the probe trigger delay $\tau_{2}=0 \mathrm{ps}$ when the electric field of the THz probe pulse exhibits the maximum amplitude (as seen in Fig. 5). $E_{\mathrm{r}}\left(\tau_{2}, \tau_{1}\right)$ and $E_{\mathrm{r}}\left(\tau_{2}\right)$ are the $\mathrm{THz}$ electric fields that are reflected from the graphene with and without photoexcitation, respectively. $\Delta E_{\mathrm{r}}\left(\tau_{1}\right) / E_{0}$ is useful for discussing the hot carrier relaxation and photoconductivity, $\Delta \sigma\left(\omega, \tau_{1}\right)=$ $\sigma\left(\omega, \tau_{1}\right)-\sigma_{0}(\omega)$, around the center frequency of the $\mathrm{THz}$ probe pulse, where $\sigma_{0}(\omega)$ is the intraband optical conductivity of graphene without pump fluence. $\Delta E_{\mathrm{r}}\left(\tau_{1}\right) / E_{0}>0$ and $\Delta E_{\mathrm{r}}\left(\tau_{1}\right) / E_{0}<0$ indicate the positive and negative photoconductivities, $\Delta \sigma_{1}\left(\omega, \tau_{1}\right)$, respectively.

We investigated the effect of the EPC on the hot carrier dynamics of photoexcited graphene on the PET substrate for different Fermi energies $\varepsilon_{\mathrm{F}}$. The parameters used in the simulation are summarized in Table I. Figures 1(a) and 1(b) depict the temporal evolutions of $T_{e}$ and $T_{\eta}$ in the heavily doped graphene with $\left|\varepsilon_{\mathrm{F}}\right|=0.43 \mathrm{eV}$ for $\left\langle D_{\mathbf{K}}^{2}\right\rangle_{\mathrm{F}}=193$ and $703(\mathrm{eV} / \AA)^{2}$ under the pump fluence $F_{0}=100 \mu \mathrm{J} / \mathrm{cm}^{2}$ calculated using the temperature model. In this case, $\left\langle D_{\Gamma}^{2}\right\rangle_{\mathrm{F}}$ is fixed at the DFT value because the EPC of the $\boldsymbol{\Gamma}_{\mathrm{LO}}$ /TO phonon is not affected by the e-e interaction and well agrees with the experiment [49]. The difference of $T_{\Gamma_{\mathrm{LO}}}$ and $T_{\Gamma_{\mathrm{TO}}}$ stems from the scattering angle dependence of $\left|D_{\lambda \boldsymbol{k} \lambda^{\prime} \boldsymbol{k}^{\prime}}^{\Gamma^{\prime}}\right|^{2}$ in Eq. (7). A comparison between Figs. 1(a) and 1(b) reveals that the rise 
TABLE I. Parameters of graphene on PET substrate and experimental setups used in the simulation. The dielectric properties of the PET substrate were obtained from Refs. [106,107].

\begin{tabular}{lccc}
\hline \hline Quantity & Lightly doped & Heavily doped \\
\hline$\left|\varepsilon_{\mathrm{F}}\right|(\mathrm{eV})$ & 0.15 & 0.43 \\
$v_{\mathrm{F}}(\mathrm{m} / \mathrm{s})$ & & $1.1 \times 10^{6}$ & \\
$\epsilon_{\mathrm{s}}$ & & 3.0 & \\
$\epsilon\left(\omega_{\mathrm{THz}}\right)$ & & 2.5 & \\
$\epsilon\left(\omega_{\text {pump }}\right)$ & & 2.4 & \\
$J_{\mathrm{a}}{ }^{\mathrm{a}}(\mathrm{eV})$ & 30,20 & & 30,20 \\
$\left\langle D_{\mathrm{\Gamma}}^{2}\right\rangle_{\mathrm{F}}(\mathrm{eV} / \AA)^{2}$ & & 45.6 & \\
$\left\langle D_{\mathrm{K}}^{2}\right\rangle_{\mathrm{F}}(\mathrm{eV} / \AA)^{2}$ & & $92.0-703$ & \\
$\rho_{\mathrm{s}}(\Omega)$ & & 100 & \\
$n_{\mathrm{i}}{ }^{\mathrm{a}}\left(10^{12} \mathrm{~cm}^{-2}\right)$ & $0,0.17$ & & $0,1.7$ \\
$\sigma_{\mathrm{dc}}\left(G_{0}\right)$ & $25.5,25.5$ & & $25.7,25.9$ \\
$\tau_{\mathrm{ph}}(\mathrm{ps})$ & & 1.0 & \\
$\tau_{\text {ie }}(\mathrm{fs})$ & & 100 & \\
$F_{0}\left(\mu \mathrm{J} / \mathrm{cm}^{2}\right)$ & & 100 & \\
$2 \tau_{\text {pump }}(\mathrm{fs})$ & & 220 & \\
$2 \tau_{\text {prob }}(\mathrm{fs})$ & & 300 & \\
\hline \hline
\end{tabular}

${ }^{\text {a The }} J_{\mathrm{a}}$ and $n_{\mathrm{i}}$ values were chosen to give nearly equal dc conductivity $\sigma_{\mathrm{dc}}$.

and relaxation dynamics of the hot carrier and optical phonon temperatures depend significantly on $\left\langle D_{\mathbf{K}}^{2}\right\rangle_{\mathrm{F}}$. At $\left\langle D_{\mathbf{K}}^{2}\right\rangle_{\mathrm{F}}=703$ $(\mathrm{eV} / \AA)^{2}, T_{\mathbf{K}}$ followed $T_{e}$ more rapidly and increases up to $1800 \mathrm{~K}$, much higher than $T_{\Gamma_{\mathrm{LO} / \mathrm{TO}}}$, indicating that substantially more hot carrier energy is mainly transferred into the $\mathbf{K}$ phonon owing to the stronger EPC. As a result, the maximum $T_{e}$ for $\left\langle D_{\mathbf{K}}^{2}\right\rangle_{\mathrm{F}}=703(\mathrm{eV} / \AA)^{2}$ becomes lower than that for $\left\langle D_{\mathbf{K}}^{2}\right\rangle_{\mathrm{F}}=193(\mathrm{eV} / \AA)^{2}$. Figure 1(c) presents the $\left\langle D_{\mathbf{K}}^{2}\right\rangle_{\mathrm{F}}$ dependence of the transient reflection change $\Delta E_{\mathrm{r}}\left(\tau_{1}\right) / E_{0}$ calculated from the $\sigma\left(\omega, \tau_{1}\right)$ using the $\mathrm{THz}$ probe pulse with
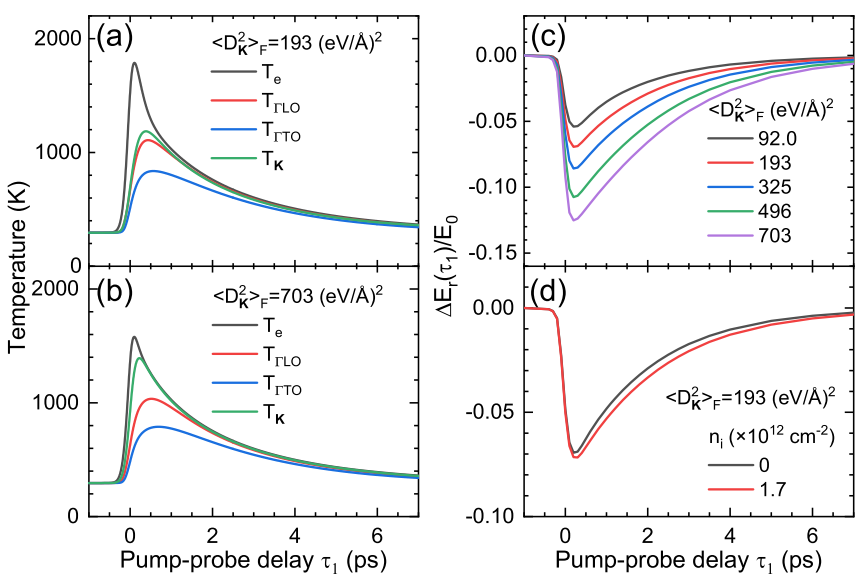

FIG. 1. Simulation results of heavily doped graphene with $\left|\varepsilon_{F}\right|=$ $0.43 \mathrm{eV}$ for $F_{0}=100 \mu \mathrm{J} / \mathrm{cm}^{2}$. Temporal evolutions of $T_{e}$ and $T_{\eta}$ for (a) $\left\langle D_{\mathbf{K}}^{2}\right\rangle_{\mathrm{F}}=193$ and (b) $\left\langle D_{\mathbf{K}}^{2}\right\rangle_{\mathrm{F}}=703(\mathrm{eV} / \AA)^{2}$. (c) $\left\langle D_{\mathbf{K}}^{2}\right\rangle_{\mathrm{F}}$ dependence of $\Delta E_{\mathrm{r}}\left(\tau_{1}\right) / E_{0}$ of graphene calculated using temporal waveforms of the $\mathrm{THz}$ probe pulse expressed by the second derivative of the Gaussian function $\exp \left(-t^{2} / \tau_{\text {pump }}^{2}\right)$ with pulse durations of $2 \tau_{\text {pump }}=300$ fs. (d) $\Delta E_{\mathrm{r}}\left(\tau_{1}\right) / E_{0}$ at $\left\langle D_{\mathbf{K}}^{2}\right\rangle_{\mathrm{F}}=193(\mathrm{eV} / \AA)^{2}$ for $n_{\mathrm{i}}=0$ and $1.7 \times 10^{12} \mathrm{~cm}^{-2}$.
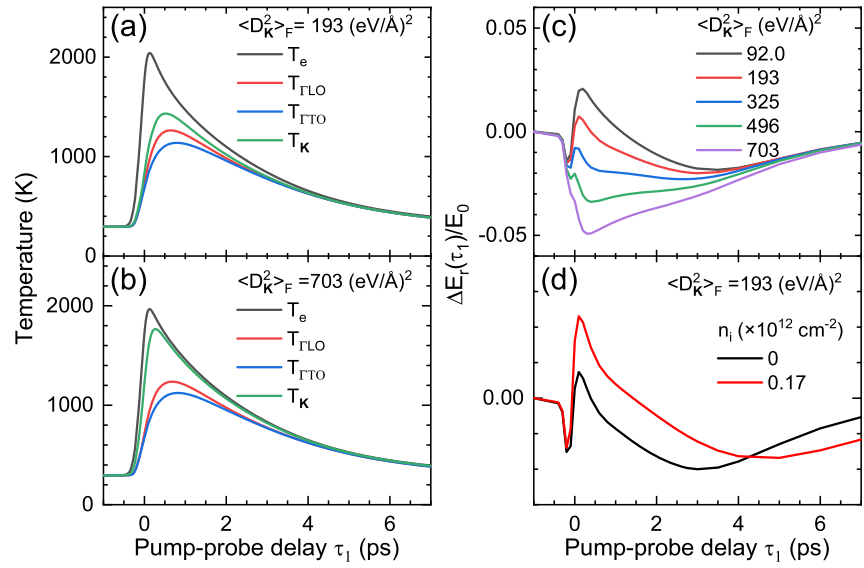

FIG. 2. Simulation results of lightly doped graphene with $\left|\varepsilon_{F}\right|=$ $0.15 \mathrm{eV}$. Temporal evolutions of $T_{e}$ and $T_{\eta}$ for (a) $\left\langle D_{\mathrm{K}}^{2}\right\rangle_{\mathrm{F}}=92.0$ and (b) $\left\langle D_{\mathbf{K}}^{2}\right\rangle_{\mathrm{F}}=703(\mathrm{eV} / \AA)^{2}$ for $F_{0}=100 \mu \mathrm{J} / \mathrm{cm}^{2}$. (c) $\left\langle D_{\mathbf{K}}^{2}\right\rangle_{\mathrm{F}}$ dependence of $\Delta E_{\mathrm{r}}\left(\tau_{1}\right) / E_{0}$ of graphene. (d) $\Delta E_{\mathrm{r}}\left(\tau_{1}\right) / E_{0}$ at $\left\langle D_{\mathbf{K}}^{2}\right\rangle_{\mathrm{F}}=193$ $(\mathrm{eV} / \AA)^{2}$ for $n_{\mathrm{i}}=0$ and $0.17 \times 10^{12} \mathrm{~cm}^{-2}$.

$2 \tau_{\mathrm{p}}=300 \mathrm{fs}$. The sign of $\Delta E_{\mathrm{r}}\left(\tau_{1}\right) / E_{0}$ remains negative indicating the negative photoconductivity as varying the $\left\langle D_{\mathbf{K}}^{2}\right\rangle_{\mathrm{F}}$. The peak value of $\left|\Delta E_{\mathrm{r}}\left(\tau_{1}\right) / E_{0}\right|$ increases monotonically as $\left\langle D_{\mathbf{K}}^{2}\right\rangle_{\mathrm{F}}$ increases and effectively reflects the enhancement of $T_{\mathbf{K}}$.

Figure 2 depicts the simulation results on the lightly doped graphene with $\left|\varepsilon_{\mathrm{F}}\right|=0.15 \mathrm{eV}$. Although the same phonon decay time $\tau_{\mathrm{ph}}=1 \mathrm{ps}$ is used, the relaxation time of $T_{e}$ of the lightly doped graphene is longer than that of the heavily doped graphene owing to the weaker $R_{\eta}^{\text {Net }}$ originating from the small density of states at the Fermi energy $\varepsilon_{\mathrm{F}}$. The sign of $\Delta E_{\mathrm{r}}\left(\tau_{1}\right) / E_{0}$ indicated in Fig. 2(c) changes depending on $\left\langle D_{\mathbf{K}}^{2}\right\rangle_{\mathrm{F}}$ in contrast to the heavily doped graphene. For a small $\left\langle D_{\mathbf{K}}^{2}\right\rangle_{\mathrm{F}}=92.0(\mathrm{eV} / \AA)^{2}, \Delta E_{\mathrm{r}}\left(\tau_{1}\right) / E_{0}$ exhibits positive photoconductivity, which is transformed into negative photoconductivity as $\left\langle D_{\mathbf{K}}^{2}\right\rangle_{\mathrm{F}}$ increases.

The different behaviors in $\Delta E_{\mathrm{r}}\left(\tau_{1}\right) / E_{0}$ between the heavily and lightly doped graphene can be understood by considering the temperature dependence of the Drude weight $D\left(T_{e}\right)$ of the graphene 2D MDF, which is the oscillator strength of free carrier absorption and plays a crucial role in carrier screening. As can be observed in Fig. 3(a), the chemical potential $\mu\left(T_{e}\right)$ of graphene 2D MDF decreases with $T_{e}$, leading to the unique temperature dependence of $D\left(T_{e}\right)$ according to $\varepsilon_{\mathrm{F}}[36,117-$ 119]. In the case of a constant carrier relaxation rate, $D\left(T_{e}\right)$ of
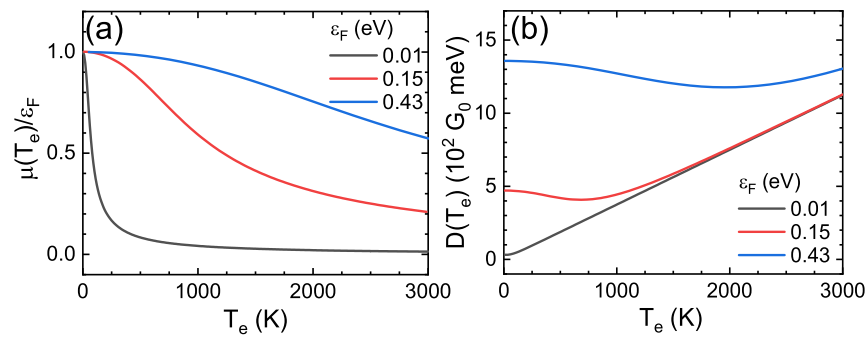

FIG. 3. $T_{e}$ dependence of (a) chemical potential $\mu\left(T_{e}\right)$ and (b) Drude weight $D\left(T_{e}\right)$ of graphene with $\varepsilon_{\mathrm{F}}=0.01,0.15$, and $0.43 \mathrm{eV}$ 
2D MDF in equilibrium is analytically expressed as

$$
D\left(T_{e}\right)=\frac{2 e^{2}}{\hbar^{2}} k_{\mathrm{B}} T_{e} \ln \left[2 \cosh \left(\frac{\mu\left(T_{e}\right)}{2 k_{\mathrm{B}} T_{e}}\right)\right] .
$$

The Drude weight of hot carriers exhibits a strong frequency dependence due to the variation in the carrier distribution and scattering rate during $\mathrm{THz}$ probing [43]. In our experimental setup, the $\Delta E_{\mathrm{r}}\left(\tau_{1}\right) / E_{0}$ measured using the broadband $\mathrm{THz}$ probe reflects the averaged Drude weight, which is proportional to $D\left(T_{e}\right)$ in Eq. (13). The $D\left(T_{e}\right)$ of the undoped graphene with $\left|\varepsilon_{\mathrm{F}}\right|=0.01 \mathrm{eV}$ in Fig. 3(b) increases linearly with $T_{e}$, yielding positive photoconductivity. However, $D\left(T_{e}\right)$ of the heavily doped graphene with $\left|\varepsilon_{\mathrm{F}}\right|=0.43 \mathrm{eV}$ decreases slightly as $T_{e}$ increases and exhibits the minimum at around $T_{e}=2000 \mathrm{~K}$, contributing to the negative photoconductivity below $T_{e}=3000 \mathrm{~K}$. At temperatures below $3000 \mathrm{~K}$, the maximum change in $D\left(T_{e}\right)$ is only $13 \%$, and the temperature dependence of $\mathrm{THz}$ conductivity change is mainly dominated by the carrier scattering with the SCOPs. In the lightly doped graphene, $D\left(T_{e}\right)$ exhibits its minimum value at around $T_{e}=$ $750 \mathrm{~K}$ and increases significantly above $T_{e}=1000 \mathrm{~K}$. The contributions of $D\left(T_{e}\right)$ and the carrier scattering with SCOPs to the photoconductivity compete with each other, resulting in either positive or negative photoconductivity, depending on $T_{e}$ and $\left\langle D_{\mathbf{K}}^{2}\right\rangle_{\mathrm{F}}$. A similar behavior has been reported in Ref. [36].

We also investigated the effect of the charged impurity on the hot carrier dynamics in the heavily and lightly doped graphene because the charged impurity is one of the dominant scattering mechanisms in graphene on substrate $[68,69,78,91]$. Here, the effective coupling constant $J_{\mathrm{a}}$ of an acoustic phonon is selected so that the dc conductivity is almost equal as shown in Table I. Figure 1(d) shows that the $\Delta E_{\mathrm{r}}\left(\tau_{1}\right) / E_{0}$ of the heavily doped graphene is almost unaffected by charged impurity scattering owing to the strong carrier screening effect. However, the $\Delta E_{\mathrm{r}}\left(\tau_{1}\right) / E_{0}$ of the lightly doped graphene in Fig. 2(d) changes significantly in the presence of the low charged impurity concentration $n_{\mathrm{i}}=$ $0.17 \times 10^{12} \mathrm{~cm}^{-2}$. The carrier-scattering rate by charged impurities increases (decreases) for $T_{e} \leqslant 1000 \mathrm{~K}\left(T_{e} \geqslant 1000 \mathrm{~K}\right)$ due to the change in the carrier screening effect; this leads to a higher positive peak with charged impurities. Therefore the information of the accurate charged impurity concentration is required to derive the $\left\langle D_{\mathbf{K}}^{2}\right\rangle_{\mathrm{F}}$ from $\Delta E_{\mathrm{r}}\left(\tau_{1}\right) / E_{0}$ of lightly doped graphene. These findings indicate that heavily doped graphene is suitable for the determination of $\left\langle D_{\mathbf{K}}^{2}\right\rangle_{\mathrm{F}}$ from $\Delta E_{\mathrm{r}}\left(\tau_{1}\right) / E_{0}$.

\section{EXPERIMENTAL RESULTS}

The graphene sample (Graphene Platform) that was examined in this study was prepared using chemical vapor deposition. The single-layer graphene (area: $10 \times 10 \mathrm{~mm}$ ) was transferred to a PET substrate. Raman scattering measurements confirmed the single-layer thickness of the sample and their low defect density. The equilibrium $\mathrm{THz}$ conductivity of the sample at room temperature $\left(T_{0}=295 \mathrm{~K}\right)$ was characterized by ultrabroadband $\mathrm{THz}$ time domain spectroscopic ellipsometry (THz-TDSE) (see Appendix A for details), which enabled the broad Drude peak to be captured directly by measuring the ratio of the reflection coefficient
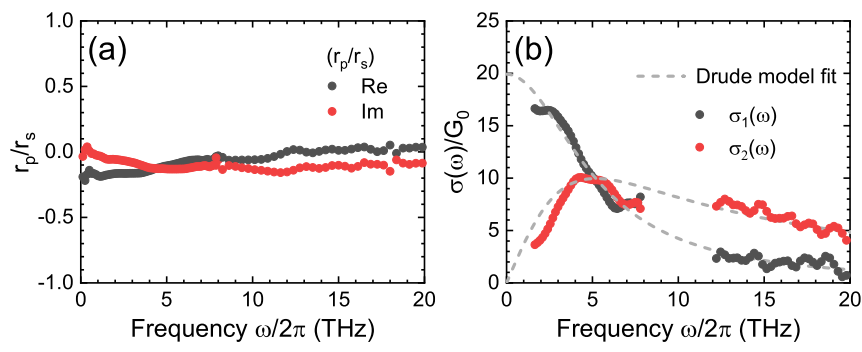

FIG. 4. (a) $\left(r_{\mathrm{p}} / r_{\mathrm{s}}\right)$ of graphene at equilibrium measured by THzTDSE. (b) $\sigma(\omega)$ at equilibrium. The dashed curves are the fitting curves of the simple Drude model.

$r_{\mathrm{p}}(\omega) / r_{\mathrm{s}}(\omega)$ in the frequency range between 1.0 and $20 \mathrm{THz}$ [120], as illustrated in Fig. 4. The fitting of the THz conductivity spectrum obtained from $r_{\mathrm{p}}(\omega) / r_{\mathrm{s}}(\omega)$ by the Drude model allows us to determine the Drude weight $D_{0}$ and carrier relaxation rate $\Gamma_{0}$ for the equilibrium state at room temperature $T_{0}=295 \mathrm{~K}$ accurately. We estimated $D_{0}=1.36 \times$ $10^{3} G_{0}$ and $\Gamma_{0}=21.4 \mathrm{meV}$, respectively. Here, $G_{0}=2 e^{2} / h$ is the quantum conductance. The corresponding Fermi energy is $\left|\varepsilon_{\mathrm{F}}\right|=0.43 \mathrm{eV}$, indicating that the sample is heavily doped and suitable for estimating the EPC strength. The carrier concentration $n_{\mathrm{c}}$ at $T_{e}=0 \mathrm{~K}$ and the dc conductivity at $T_{0}$ were estimated as $n_{\mathrm{c}}=1.1 \times 10^{13} \mathrm{~cm}^{-2}$ and $\sigma_{\mathrm{dc}}=20 G_{0}$, respectively, where we used $v_{\mathrm{F}}=1.1 \times 10^{6} \mathrm{~m} / \mathrm{s}$ considering the carrier and dielectric screening effect in heavily doped graphene on PET substrate [121]. Figure 5(a) presents the optical setup of the reflection-type OPTP used in the experiment. Amplified femtosecond laser pulses $(1 \mathrm{kHz}$ repetition rate, $785 \mathrm{~nm}$ center wavelength) are used to generate ultrabroadband $\mathrm{THz}$ probe pulses from laser-excited air plasma [122]. The s-polarized pump pulses with a pulse duration of
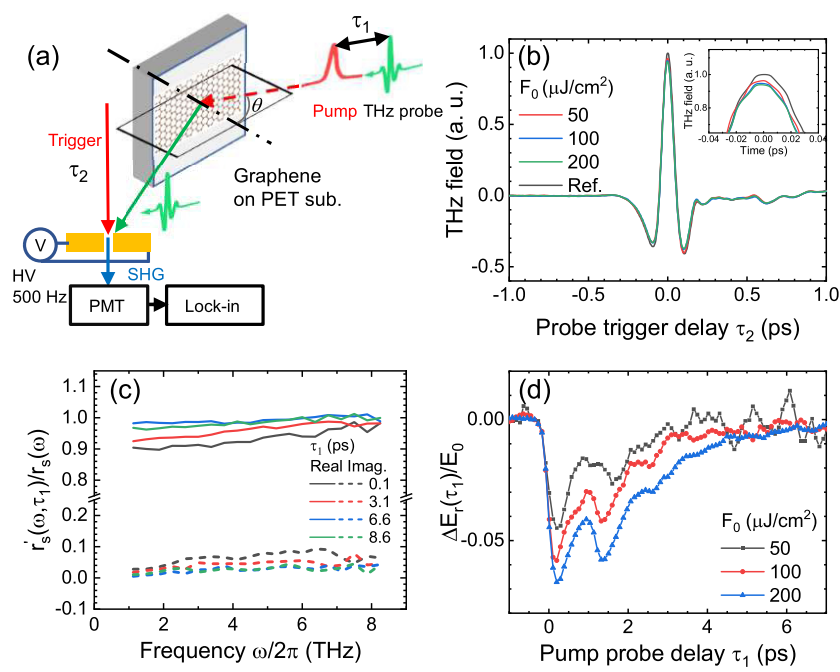

FIG. 5. (a) Schematic of reflection-type OPTP setup. Pump, pump pulse; Trigger, trigger pulse; SHG, second-harmonic generation; HV, high voltage; PMT, photomultiplier tube; sub., substrate. (b) Temporal waveforms of THz probe pulse measured at $\tau_{1}=0.1$ ps. (c) Frequency dependence of $\left[r_{\mathrm{s}}^{\prime}\left(\omega, \tau_{1}\right) / r_{\mathrm{s}}(\omega)\right]$ at $\tau_{1}=0.1,3.1$, 6.6, and $8.6 \mathrm{ps}$ at $F_{0}=200 \mu \mathrm{J} / \mathrm{cm}^{2}$. (d) Pump fluence dependence of $\Delta E\left(\tau_{1}\right) / E_{0}$. 


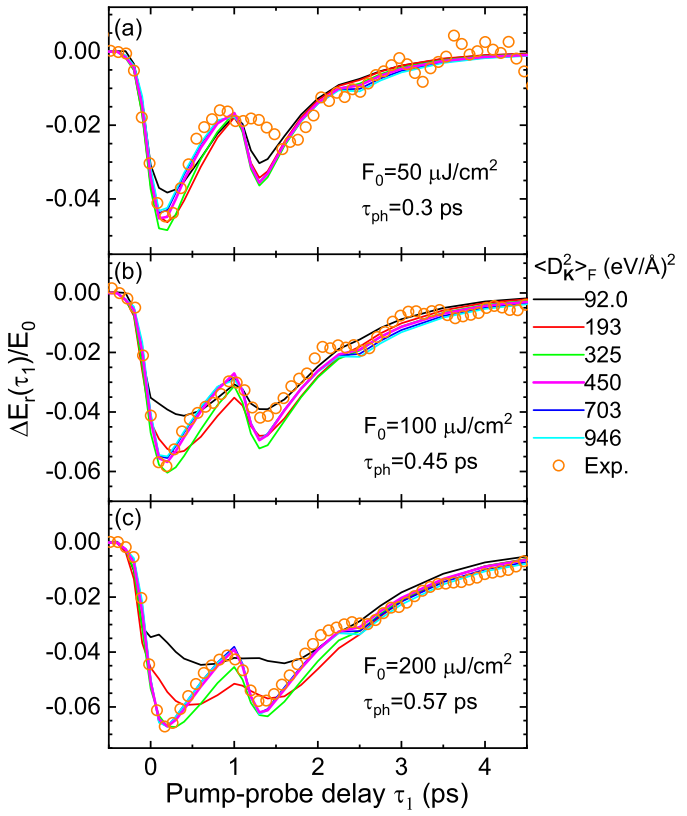

FIG. 6. Comparison of $\Delta E_{\mathrm{r}}\left(\tau_{1}\right) / E_{0}$ between experiment and calculations for different EPCs for pump fluence (a $F_{0}=50$, (b) $F_{0}=100$, and (c) $F_{0}=200 \mu \mathrm{J} / \mathrm{cm}^{2}$. The red open circles represent the experimental $\Delta E_{\mathrm{r}}\left(\tau_{1}\right) / E_{0}$. The solid curves correspond to the $\Delta E_{\mathrm{r}}\left(\tau_{1}\right) / E_{0}$ calculated using $\left\langle D_{\Gamma}^{2}\right\rangle_{\mathrm{F}}=92.0$ (DFT, black), 193 (GW, red), 325 (green), 450 (magenta), 703 (dark blue), and 946 (light blue) $(\mathrm{eV} / \AA)^{2}$, respectively, under the maximum $J_{\mathrm{sc}}$ condition.

$220 \mathrm{fs}$ are loosely focused and excited the graphene sample at an incident angle of $\theta=60^{\circ}$, and the created hot carrier state was probed by s-polarized $\mathrm{THz}$ pulses with a pump probe time delay $\tau_{1}$. The temporal waveforms of the reflected THz probe pulses are measured by air breakdown coherent detection, which detects the second-harmonic generation of the trigger pulse induced by the THz electric field [123]. Figure 5(b) depicts the temporal waveforms of the THz probe pulse reflected from the photoexcited graphene. When the pump fluence is increased, the peak amplitude of $\mathrm{THz}$ probe decreases slightly, indicating negative photoconductivity. The ratio of the reflection coefficient $r_{\mathrm{s}}^{\prime}\left(\omega, \tau_{1}\right) / r_{\mathrm{s}}(\omega)$ of graphene with and without pump fluence $F_{0}=200 \mu \mathrm{J} / \mathrm{cm}^{2}$ calculated by Fourier transformation of the $\mathrm{THz}$ waveforms at different $\tau_{1}$ values, as plotted in Fig. 5(c), decreases and then recovers to equilibrium reflecting the rise and subsequent relaxation process of the hot carrier dynamics, and this was used for the calculation of $\sigma\left(\omega, \tau_{1}\right)$ (see Appendix B for details). Figure 5(d) presents the fluence dependence of $\Delta E_{\mathrm{r}}\left(\tau_{1}\right) / E_{0}$, which exhibits multiple negative peaks around $\tau_{1}=0.2,1.4,2.3 \mathrm{ps}$ owing to the multiple reflections inside the PET substrate. As $F_{0}$ increases, the peak height $\Delta E_{\mathrm{r}}\left(\tau_{1}\right) / E_{0}$ increases, but it exhibits saturation behavior with an increased relaxation time.

To determine the EPC constant $\left\langle D_{\mathbf{K}}^{2}\right\rangle_{\mathrm{F}}$, we compared the experimentally determined $\Delta E_{\mathrm{r}}\left(\tau_{1}\right) / E_{0}$ with the corresponding calculation; we assumed the minimum and maximum energy loss rates $J_{\mathrm{sc}}$ in the defect-induced SC carrier-cooling process because the SC and the optical phonon emission dominate the hot-carrier-cooling process. Figures 6(a)-6(c) depict
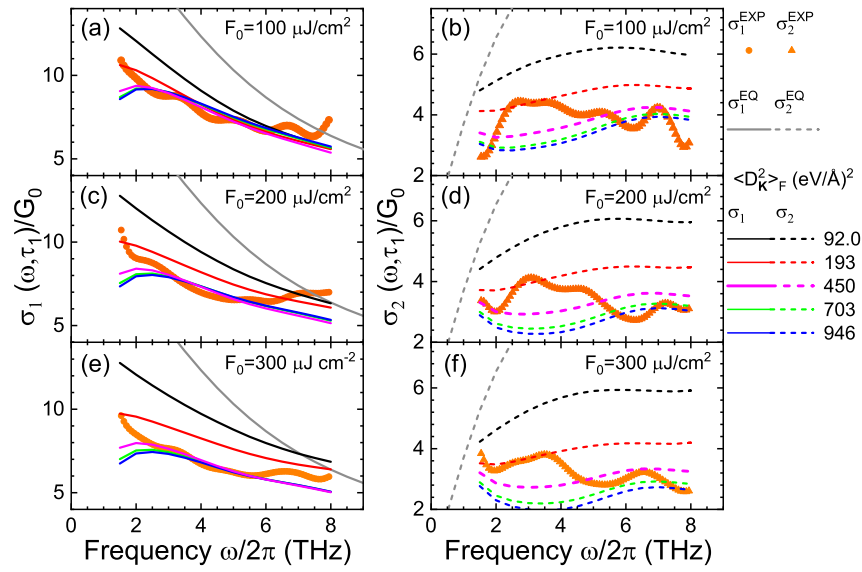

FIG. 7. Pump fluence dependence of $\sigma\left(\omega, \tau_{1}\right)=\sigma_{1}\left(\omega, \tau_{1}\right)+$ $i \sigma_{2}\left(\omega, \tau_{1}\right)$ (orange symbols) of heavily doped graphene obtained from for (a) and (b) $F_{0}=100$, (c) and (d) $F_{0}=200$, and (e) and (f) $F_{0}=300 \mu \mathrm{J} / \mathrm{cm}^{2}$. The gray solid and dashed curves correspond to the fitting curve of $\sigma\left(\omega, \tau_{1}\right)$ at equilibrium shown in Fig. 4(b). The black, red, magenta, green, and blue curves correspond to $\sigma\left(\omega, \tau_{1}\right)$ at $\tau_{1}=0.1 \mathrm{ps}$ calculated using $\left\langle D_{\mathbf{K}}^{2}\right\rangle_{\mathrm{F}}=92.0,193,450,703$, and $946\left(\mathrm{eV} \AA^{-1}\right)^{2}$, respectively, under the maximum $J_{\text {sc }}$ condition.

the experimentally determined and calculated $\Delta E_{\mathrm{r}}\left(\tau_{1}\right) / E_{0}$ values with the maximum $J_{\mathrm{sc}}$, where $J_{\mathrm{a}}=30 \mathrm{eV}$ and $\rho_{\mathrm{s}}=$ $100 \Omega$. The calculated $\Delta E_{\mathrm{r}}\left(\tau_{1}\right) / E_{0}$ is significantly dependent on $\left\langle D_{\mathbf{K}}^{2}\right\rangle_{\mathrm{F}}$ and the pump fluence $F_{0}$. For the $\left\langle D_{\mathbf{K}}^{2}\right\rangle_{\mathrm{F}}$ determined through the DFT and GW calculations, the peak height and temporal evolution of $\Delta E_{\mathrm{r}}\left(\tau_{1}\right) / E_{0}$ differ significantly from the experimental values; higher values of $\left\langle D_{\mathrm{K}}^{2}\right\rangle_{\mathrm{F}}=450-946$ $(\mathrm{eV} / \AA)^{2}$ are required to reproduce $\Delta E_{\mathrm{r}}\left(\tau_{1}\right) / E_{0}$.

Figure 7 presents the pump fluence dependence of $\sigma\left(\omega, \tau_{1}\right)$ measured at $\tau_{1}=0.1 \mathrm{ps}$ when the maximum negative photoconductivity appears. We observe a significant reduction in the $\mathrm{THz}$ conductivity; this indicates that a large negative photoconductivity with non-Drude behavior is attained as $F_{0}$ increases and $\sigma\left(\omega, \tau_{1}\right)$ for $F_{0}=200 \mu \mathrm{J} / \mathrm{cm}^{2}$ reaches less than half the corresponding values at equilibrium (gray curve). Thus a significant increase in the carrier scattering by SCOPs is considered to occur at high temperatures. It is observed that the $\sigma\left(\omega, \tau_{1}\right)$ values for $\left\langle D_{\Gamma}^{2}\right\rangle_{\mathrm{F}}$ determined based on the DFT (black curve) and GW (blue curve) calculations cannot reproduce the observed negative photoconductivity, even if the SA effect is not considered. In contrast, the $\sigma\left(\omega, \tau_{1}\right)$ for $\left\langle D_{\Gamma}^{2}\right\rangle_{\mathrm{F}}=703$ and $946(\mathrm{eV} / \AA)^{2}$ exhibit a larger deviation than that for $\left\langle D_{\Gamma}^{2}\right\rangle_{\mathrm{F}}=450(\mathrm{eV} / \AA)^{2}$ does. From the comparison of $\sigma\left(\omega, \tau_{1}\right)$ and $\Delta E_{\mathrm{r}}\left(\tau_{1}\right) / E_{0}$ with the calculated values shown in Figs. 6 and 7 , we estimated $\left\langle D_{\mathbf{K}}^{2}\right\rangle_{\mathrm{F}} \approx 450 \pm 45(\mathrm{eV} / \AA)^{2}$ and $\tau_{\mathrm{ie}}=116 \mathrm{fs}$. At these values, the calculations (magenta curves in Fig. 6 and 7) with the maximum $J_{\mathrm{sc}}$ show the best fit with the experimental results. Note that the comparison using the calculation with the minimum $J_{\mathrm{sc}}$ where $J_{\mathrm{a}}=10 \mathrm{eV}$ and $\rho_{\mathrm{s}}=40 \Omega$ yields the same EPC constant as that shown in Appendix F. From the comparison, we obtained $\tau_{\text {ie }}=116$ and $121 \mathrm{fs}$ with the maximum and minimum $J_{\mathrm{sc}}$ corresponding to the saturated pump intensity $I_{s}=1.7 \times 10^{8}$ and $1.6 \times 10^{8}$ $\mathrm{W} / \mathrm{cm}^{2}$ for $A_{12}^{\mathrm{s} *}$, respectively; the aforementioned values are slightly smaller than those reported in Refs. [111,124]. 


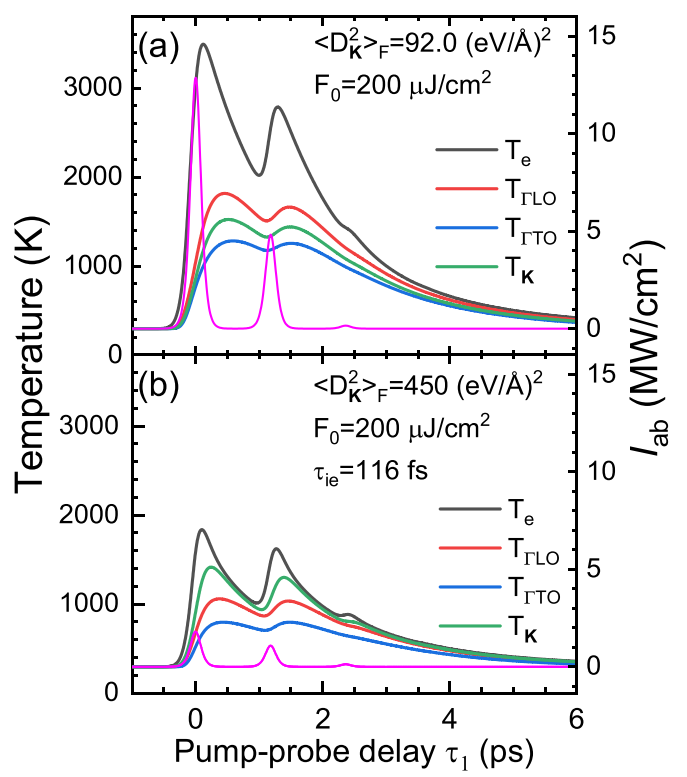

FIG. 8. Temporal evolution of $T_{e}$ and $T_{\eta}$ calculated for (a) $\left\langle D_{\mathbf{K}}^{2}\right\rangle_{\mathrm{F}}=92.0$ and (b) $\left\langle D_{\mathbf{K}}^{2}\right\rangle_{\mathrm{F}}=450(\mathrm{eV} / \AA)^{2}$ under the maximum $J_{\text {sc }}$ condition. The magenta curve is the absorbed pump intensity $\mathcal{I}_{\mathrm{ab}}$, calculated considering the SA effect.

Figure 8 presents the temporal evolution of $T_{e}$ and $T_{\eta}$ calculated for $\left\langle D_{\mathbf{K}}^{2}\right\rangle_{\mathrm{F}}=92.0$ and $450(\mathrm{eV} / \AA)^{2}$ under the pump fluence $F_{0}=200 \mu \mathrm{J} / \mathrm{cm}^{2}$ indicating that hot carrier and phonon dynamics are significantly dependent on the EPC. For $\left\langle D_{\mathbf{K}}^{2}\right\rangle_{\mathrm{F}}=92.0(\mathrm{eV} / \AA)^{2}$ as shown in Fig. 8(a), the hot carrier temperature increases beyond $T_{e}=3000 \mathrm{~K}$, and $T_{\mathbf{K}}$ follows $T_{e}$ slowly owing to the weak EPC and reaches up to $T_{\mathbf{K}} \approx 1500 \mathrm{~K}$. In this high temperature range, the carrier scattering by optical phonons is dominant, and the Drude weight $D\left(T_{e}\right)$ makes a positive contribution to $\sigma\left(\omega, \tau_{1}\right)$ in contrast to the carrier scattering. The competition of these factors leads to broader peaks of $\Delta E_{\mathrm{r}}\left(\tau_{1}\right) / E_{0}$ for DFT (black curve) in Fig. 7(c) than those of $T_{\eta}$ in Fig. 8(a). For $\left\langle D_{\mathbf{K}}^{2}\right\rangle_{\mathrm{F}}=$ $450(\mathrm{eV} / \AA)^{2}$ as seen in Fig. 8(b), the hot carrier temperature increases up to only $T_{e} \approx 2000 \mathrm{~K}$, and $T_{\mathbf{K}}$ follows $T_{e}$ rapidly and reaches up to $T_{\mathbf{K}} \approx 1400 \mathrm{~K}$ owing to the SA effect and strong EPC. In this case, $D\left(T_{e}\right)$ makes the same contribution to $\sigma\left(\omega, \tau_{1}\right)$ as the optical phonon scattering, resulting in sharper peaks of $\Delta E_{\mathrm{r}}\left(\tau_{1}\right) / E_{0}$ and a successful reproduction of the experimental results. Furthermore, the frequency dependence of $\sigma\left(\omega, \tau_{1}\right)$ at $\tau_{1}=0.1 \mathrm{ps}$ in Fig. 6 deviates from the simple Drude model as $F_{0}$ increases. This originates from the rapid temporal variation in the carrier temperature and scattering rate during the $\mathrm{THz}$ probing time following the photoexcitation, and the calculation with $\left\langle D_{\mathbf{K}}^{2}\right\rangle_{\mathrm{F}}=450(\mathrm{eV} / \AA)^{2}$ effectively reproduces the observed large negative photoconductivity with non-Drude behavior. This indicates that most photoexcited carriers are recombined and the quasiequilibrium hot carrier state is almost established at $\tau_{1}=0.1 \mathrm{ps}$ owing to the strong Auger recombination in the heavily doped graphene, as reported in Ref. [26]. The parameters used in the calculation are displayed in Table II.
TABLE II. Parameters used in calculation of $\Delta E_{\mathrm{r}}\left(\tau_{1}\right) / E_{0}$, $\sigma\left(\omega, \tau_{1}\right)$, and $T_{e}$ in Figs. 6, 7, and 8, respectively, under the maximum $J_{\mathrm{sc}}$ condition. The values of $\left\langle D_{\Gamma}^{2}\right\rangle_{\mathrm{F}}, J_{\mathrm{a}}$, and $\rho_{\mathrm{s}}$ are set to $\left\langle D_{\Gamma}^{2}\right\rangle_{\mathrm{F}}=45.6$ $(\mathrm{eV} / \AA)^{2}, J_{\mathrm{a}}=30.0 \mathrm{eV}$, and $\rho_{\mathrm{s}}=40.0 \Omega$, respectively. The charged impurity concentration $n_{\mathrm{i}}$ is selected to provide the same dc conductivity $\sigma_{\mathrm{dc}}=20.0 G_{0}$ at equilibrium for $T_{0}=295 \mathrm{~K}$.

\begin{tabular}{llccc}
\hline \hline$\left\langle D_{\mathrm{K}}^{2}\right\rangle_{\mathrm{F}}(\mathrm{eV} / \AA)^{2}$ & $\tau_{\text {ie }}(\mathrm{fs})$ & $I_{\mathrm{s}}\left(\mathrm{W} / \mathrm{cm}^{2}\right)$ & $n_{\mathrm{i}}\left(\mathrm{cm}^{-2}\right)$ & $\lambda_{\mathbf{K}}\left(\varepsilon_{\mathrm{F}}\right)$ \\
\hline 92.0 & & & $1.15 \times 10^{12}$ & 0.02 \\
193 & & & $1.13 \times 10^{12}$ & 0.04 \\
450 & 116 & $1.72 \times 10^{8}$ & $1.09 \times 10^{12}$ & 0.09 \\
703 & 210 & $0.53 \times 10^{8}$ & $1.05 \times 10^{12}$ & 0.14 \\
946 & 299 & $0.26 \times 10^{8}$ & $1.01 \times 10^{12}$ & 0.19 \\
\hline \hline
\end{tabular}

\section{DISCUSSION}

Based on the fitting of $\Delta E_{\mathrm{r}}\left(\tau_{1}\right) / E_{0}$ by the calculation considering the EPC, we estimated the phenomenological phonon decay time due to lattice anharmonicity as $\tau_{\mathrm{ph}}=$ $0.3,0.45$, and $0.57 \mathrm{ps}$ for $F_{0}=50,100$, and $200 \mu \mathrm{J} / \mathrm{cm}^{2}$, respectively, under the maximum $J_{\text {sc }}$ condition. References $[125,126]$ reported longer $\tau_{\mathrm{ph}}=0.8-1.5 \mathrm{ps}$ for graphene on a $\mathrm{SiO}_{2}$ substrate. However, these values were determined from the simple fitting of transient absorption or anti-Stokes Raman intensity by an exponential function and do not consider the EPC. The simple fitting of $\Delta E_{\mathrm{r}}\left(\tau_{1}\right) / E_{0}$ with an exponential curve results in $\tau_{\mathrm{ph}}=1.15-1.5 \mathrm{ps}$, which is comparable to the reported values. A theoretical study reported the phonon decay times $\tau_{\mathrm{ph}} \approx 3.5$ and $4.5 \mathrm{ps}$ for $\boldsymbol{\Gamma}$ and $\mathbf{K}$ phonons by only considering the anharmonicity of the lattice in graphene without substrate [105]. Therefore the obtained $\tau_{\mathrm{ph}}$ indicates the dominant contribution of the substrate for the optical phonon decay channel.

The dimensionless coupling constants $\lambda_{\Gamma}$ and $\lambda_{K}$ for the optical phonons near the $\boldsymbol{\Gamma}$ and $\mathbf{K}$ points, respectively, are useful for comparing the EPC strengths determined from various experiments and calculations, which are defined as [49]

$$
\lambda_{\Gamma, \mathbf{K}}=\frac{F_{\Gamma, \mathbf{K}}^{2} A_{\text {u.c. }}}{2 M \hbar \omega_{\Gamma, \mathbf{K}} v_{F}^{2}} .
$$

In the above, $M \approx 2.00 \times 10^{-26} \mathrm{~kg}$ is the mass of the carbon atom, and $A_{\text {u.c. }} \approx 5.24 \AA^{2}$ is the unit-cell area. $F_{\Gamma}^{2}$ and $F_{\mathbf{K}}^{2}$ have the dimensionality of a force and are the proportionality coefficients between the change in the effective Hamiltonian and lattice displacement along the corresponding phonon mode. Subsequently, the matching rules are expressed as $F_{\Gamma}^{2}=4\left\langle D_{\Gamma}^{2}\right\rangle_{\mathrm{F}}$ and $F_{\mathbf{K}}^{2}=2\left\langle D_{\mathbf{K}}^{2}\right\rangle_{\mathrm{F}}$. Note that $\lambda_{\mathbf{K}}$ is subject to Coulomb renormalization, which implies that $\lambda_{\mathbf{K}}$ is dependent on the electronic energy scale, such as the electron energy, Fermi energy, or temperature $T$, whichever is larger: $\lambda_{\mathbf{K}}=\lambda_{\mathbf{K}}\left(\max \left\{|\varepsilon|,\left|\varepsilon_{\mathrm{F}}\right|,|T|\right\}\right)$. From $\left\langle D_{\mathbf{K}}^{2}\right\rangle_{\mathrm{F}} \approx 450(\mathrm{eV} / \AA)^{2}$, we estimated $\lambda_{\mathbf{K}}\left(\varepsilon_{\mathrm{F}}\right) \approx 0.09$ using Eqs. (13) and (14). Figure 9 presents the flow of $\lambda_{\Gamma}$ and $\lambda_{K}$ for different background static dielectric constants $\epsilon_{\mathrm{av}}=\left(1+\epsilon_{\mathrm{s}}\right) / 2=1,2$, and 5 calculated by solving the renormalization group equation in Ref. [53], which sum up the leading logarithmic corrections and go beyond the Hartree-Fock approximation. The bare values of the dimensionless EPCs $\lambda_{\Gamma}=0.031$ and $\lambda_{K}=0.038$ 


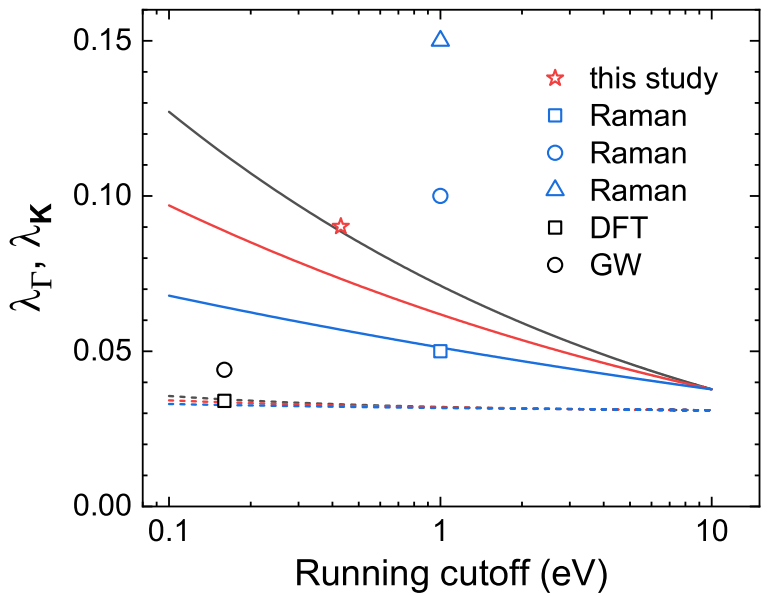

FIG. 9. Flow of dimensionless coupling constants $\lambda_{\Gamma}$ and $\lambda_{\mathbf{K}}$ (three dashed and solid curves, respectively) for three values of $\epsilon_{\mathrm{av}}=1,2$, and 5. The red and blue symbols correspond to the $\lambda_{\mathbf{K}}$ determined in this study $\left(\epsilon_{\mathrm{av}}=2\right)$ and by Raman studies $\left(\epsilon_{\mathrm{av}}=5\right)$ from Ref. [49] (blue open square and circle) and Ref. [127] (blue open triangle). The black open square and circle correspond to $\lambda_{K}$ by the DFT $[44,49]$ and GW $[47,49,55]$ calculations $\left(\epsilon_{\mathrm{av}}=1\right)$, respectively.

were selected to satisfy the relation $\lambda_{\Gamma} / \lambda_{\mathbf{K}}=\omega_{\mathbf{K}} / \omega_{\Gamma}$ and to reproduce the experimental value $\lambda_{\Gamma}=0.031$ [127]. The renormalization group analysis demonstrated that, although $\lambda_{\Gamma}$ was almost constant, $\lambda_{\mathbf{K}}$ was strongly dependent on the energy scale as well as $\epsilon_{\mathrm{av}}$. The obtained value of $\lambda_{\mathbf{K}}\left(\varepsilon_{\mathrm{F}}\right)=0.09$ is slightly larger than the calculated value of $\lambda_{\mathrm{K}}\left(\varepsilon_{\mathrm{F}}\right)=0.073$. According to the ratio $\lambda\left(\omega_{\mathbf{K}}\right) / \lambda_{\mathbf{K}}\left(\varepsilon_{\mathrm{F}}\right)=1.21$ for $\epsilon_{\mathrm{av}}=2$ in Fig. 9, we obtained $\lambda\left(\omega_{\mathbf{K}}\right)=0.11$, which is a factor of 3.2 larger than the DFT value $\lambda_{\mathbf{K}}\left(\omega_{\mathbf{K}}\right)=0.034$. Raman studies [50,51,127-129] using a field effect transistor based on the polymer electrolyte $\left(\epsilon_{\mathrm{av}}=5\right)$ reported $\lambda_{\Gamma}=0.028$ and 0.031 from the ratio of the area between the $G$ and $2 D$ peaks, which were comparable to $\lambda_{\Gamma}=0.028$ by the DFT calculation of $\left\langle g_{\Gamma}^{2}\right\rangle_{\mathrm{F}}$ using Eq. (14). However, $\lambda_{\mathbf{K}}\left(E_{\mathrm{L}} / 2\right)$ ranged between 0.05 and 0.15 as seen in Fig. 9, where $E_{L}$ is the laser excitation energy (for a typical Raman measurement $E_{\mathrm{L}} / 2 \sim 1 \mathrm{eV}$ ). The corresponding $\lambda_{\mathbf{K}}\left(\omega_{\mathbf{K}}\right)$ are estimated as 0.063 and 0.19 . The lower limit value is comparable to the calculated $\lambda_{\mathbf{K}}\left(\omega_{\mathbf{K}}\right)$ for $\epsilon_{\mathrm{av}}=5$. Although Raman spectroscopy is a powerful tool for the determination of $\lambda_{\mathbf{K}}\left(\omega_{\mathbf{K}}\right)$ as well as $\lambda_{\Gamma}\left(\omega_{\mathbf{K}}\right)$, it requires an accurate estimation of the gate capacitance of the field effect transistor (FET) device which is not required in OPTP experiments.

\section{CONCLUSION}

In conclusion, we investigated the EPC of the optical phonons near the $\mathbf{K}$ point of heavily doped graphene on PET substrate and the hot carrier dynamics using a combination of time-resolved $\mathrm{THz}$ spectroscopy and numerical simulations. The hot carrier dynamics in heavily doped graphene on PET substrate is less sensitive to the extrinsic charged impurity and surface polar phonons of the substrate and is dominated by the electron-optical phonon interactions. According to the quantitative analysis based on the BTE and a comprehensive temperature model considering the SA effect on pump fluence, the $\Delta E\left(\tau_{1}\right) / E_{0}$ value can be used for the determination of the EPC in graphene. The estimated $\left\langle D_{\mathrm{K}}^{2}\right\rangle_{\mathrm{F}} \approx 450(\mathrm{eV} / \AA)^{2}$ indicates the strong renormalization by e-e interaction and a corresponding dimensionless coupling constant $\lambda_{\mathbf{K}}\left(E_{\mathrm{F}}\right) \approx$ 0.09 slightly larger than the calculation by the renormalization group theory. The extension of the simulation model to undoped or lightly doped graphene on various substrates requiring the accurate estimation of charged impurity concentration and effect of surface polar phonons of the substrate are a future issue that will be important to the development of graphene optoelectronic devices.

\section{ACKNOWLEDGMENTS}

This work was supported by JSPS KAKENHI (Grant No. 19H01905) and Research Foundation for Opto-Science and Technology.

\section{APPENDIX A: CALCULATION OF EQUILIBRIUM OPTICAL CONDUCTIVITY OF GRAPHENE FROM THE THz TIME DOMAIN SPECTROSCOPIC ELLIPSOMETRY EXPERIMENT}

In this Appendix, we explain the calculation procedure of the $\mathrm{THz}$ conductivity $\sigma\left(\omega_{\mathrm{THz}}\right)$ of graphene on the substrate from the ratio of the complex reflection coefficient $\left[r_{\mathrm{p}}\left(\omega_{\mathrm{THz}}\right) / r_{\mathrm{s}}\left(\omega_{\mathrm{THz}}\right)\right]$ for the $\mathrm{p}$ - and s-polarized $\mathrm{THz}$ waves measured by $\mathrm{THz}$ time domain spectroscopic ellipsometry (THz-TDSE) [120]. According to the standard thin-film approximation, the reflection coefficients of graphene on a substrate for $\mathrm{p}$ - and s-polarized $\mathrm{THz}$ waves are given by [130]

$$
\begin{gathered}
r_{\mathrm{p}}\left(\omega_{\mathrm{THz}}\right)=\frac{\sigma\left(\omega_{\mathrm{THz}}\right) Z_{0}+A^{\prime}}{\sigma\left(\omega_{\mathrm{THz}}\right) Z_{0}+A}, \\
r_{\mathrm{s}}\left(\omega_{\mathrm{THz}}\right)=-\frac{\sigma\left(\omega_{\mathrm{THz}}\right) Z_{0}+B^{\prime}}{\sigma\left(\omega_{\mathrm{THz}}\right) Z_{0}+B},
\end{gathered}
$$

where

$$
\begin{aligned}
& A=\frac{\epsilon_{2}\left(\omega_{\mathrm{THz}}\right)}{\left[\epsilon_{2}\left(\omega_{\mathrm{THz}}\right)-\epsilon_{1}\left(\omega_{\mathrm{THz}}\right) \sin ^{2} \theta_{1}\right]^{1 / 2}}+\frac{\epsilon_{1}^{1 / 2}\left(\omega_{\mathrm{THz}}\right)}{\cos \theta_{1}}, \\
& A^{\prime}=\frac{\epsilon_{2}\left(\omega_{\mathrm{THz}}\right)}{\left[\epsilon_{2}\left(\omega_{\mathrm{THz}}\right)-\epsilon_{1}\left(\omega_{\mathrm{THz}}\right) \sin ^{2} \theta_{1}\right]^{1 / 2}}-\frac{\epsilon_{1}^{1 / 2}\left(\omega_{\mathrm{THz}}\right)}{\cos \theta_{1}}, \\
& B=\left[\epsilon_{2}\left(\omega_{\mathrm{THz}}\right)-\epsilon_{1}\left(\omega_{\mathrm{THz}}\right) \sin ^{2} \theta_{1}\right]^{1 / 2}+\epsilon_{1}^{1 / 2}\left(\omega_{\mathrm{THz}}\right) \cos \theta_{1} \text {, } \\
& B^{\prime}=\left[\epsilon_{2}\left(\omega_{\mathrm{THz}}\right)-\epsilon_{1}\left(\omega_{\mathrm{THz}}\right) \sin ^{2} \theta_{1}\right]^{1 / 2}-\epsilon_{1}^{1 / 2}\left(\omega_{\mathrm{THz}}\right) \cos \theta_{1} .
\end{aligned}
$$

In the above, $Z_{0}=376.7 \Omega$ is the vacuum impedance, and $\theta_{1}=60^{\circ}$ is the incidence angle of the $\mathrm{THz}$ wave. Furthermore, $\epsilon_{\mathrm{i}}\left(\omega_{\mathrm{THz}}\right)$ is the dielectric constant of layer $i$, as indicated in 


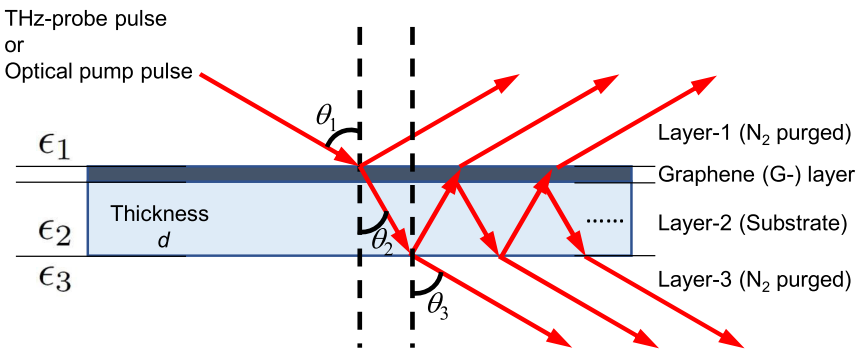

FIG. 10. Schematic of systems considered in the THz-TDSE and OPTP measurements, showing the incident $\mathrm{THz}$ probe or optical pump pulse.

Fig. 10. From Eqs. (A1a) and (A1b), $\sigma(\omega)$ is expressed as

$$
\begin{aligned}
\sigma(\omega)= & \frac{-1}{2\left[1+\rho_{\mathrm{ps}}(\omega)\right] Z_{0}}\left[\rho_{\mathrm{ps}}(\omega)\left(A+B^{\prime}\right)+A^{\prime}+B\right] Z_{0} \\
& +\left\{\left[\rho_{\mathrm{ps}}(\omega)\left(A+B^{\prime}\right)+A^{\prime}+B\right]^{2}\right. \\
& \left.\left.-4\left[1+\rho_{\mathrm{ps}}(\omega)\right]\left[\rho_{\mathrm{ps}}(\omega) A B^{\prime}+A^{\prime} B\right]\right\}^{1 / 2}\right] .
\end{aligned}
$$

Here, $\rho_{\mathrm{ps}}(\omega)=r_{\mathrm{p}} / r_{\mathrm{s}}$ measured by THz-TDSE. $\sigma\left(\omega_{\mathrm{THz}}\right)$ can be determined by substituting $\rho(\omega)$ into Eq. (A3).

\section{APPENDIX B: CALCULATION OF HOT CARRIER OPTICAL CONDUCTIVITY OF PHOTOEXCITED GRAPHENE FROM THE REFLECTION COEFFICIENT FROM THE OPTP EXPERIMENT}

In this Appendix, we present the calculation procedure of the hot carrier $\mathrm{THz}$ conductivity $\sigma\left(\omega_{\mathrm{THz}}, \tau_{1}\right)$ of photoexcited graphene at the pump probe delay $\tau_{1}$ from the reflectiontype OPTP measurement. The reflection-type OPTP measures the ratio of the complex reflection coefficient $X_{\mathrm{s}}\left(\omega_{\mathrm{THz}}, \tau_{1}\right)=$ $r_{\mathrm{s}}^{\prime}\left(\omega_{\mathrm{THz}}, \tau_{1}\right) / r_{\mathrm{s}}\left(\omega_{\mathrm{THz}}\right)$ of graphene with and without photoexcitation. The reflection coefficient for the s-polarization of graphene with complex conductivity $\sigma\left(\omega_{\mathrm{THz}}\right)$ at an incident angle of $\theta_{1}$ is expressed by Eq. (A1b). Similarly, the THz-amplitude reflection coefficient for the s-polarization of graphene with hot carrier complex conductivity $\sigma\left(\omega_{\mathrm{THz}}, \tau_{1}\right)$ on the substrate at an incident angle of $\theta_{1}$ for the pump probe delay $\tau_{1}$ is expressed by

$$
r_{\mathrm{s}}^{\prime}\left(\omega_{\mathrm{THz}}, \tau_{1}\right)=-\frac{\sigma\left(\omega_{\mathrm{THz}}, \tau_{1}\right) Z_{0}+B^{\prime}}{\sigma\left(\omega_{\mathrm{THz}}, \tau_{1}\right) Z_{0}+B} .
$$

Using Eqs. (A1b) and (B1), we obtain

$$
\sigma\left(\omega_{\mathrm{THz}}, \tau_{1}\right)=-\frac{B X_{\mathrm{s}}\left(\omega_{\mathrm{THz}}, \tau_{1}\right) r_{\mathrm{s}}\left(\omega_{\mathrm{THz}}\right)+B^{\prime}}{Z_{0}\left[1+X_{\mathrm{s}}\left(\omega_{\mathrm{THz}}, \tau_{1}\right) r_{\mathrm{s}}\left(\omega_{\mathrm{THz}}\right)\right]},
$$

where $B$ and $B^{\prime}$ are provided by Eqs. (A2c) and (A2d), respectively, and $r_{\mathrm{s}}\left(\omega_{\mathrm{THz}}\right)$ is calculated using the equilibrium $\sigma\left(\omega_{\mathrm{THz}}\right)$ obtained by THz-TDSE. We can obtain the $\sigma\left(\omega_{\mathrm{THz}}, \tau_{1}\right)$ by substituting $X_{\mathrm{s}}\left(\omega_{\mathrm{THz}}, \tau_{1}\right)$ into Eq. (B2).

\section{APPENDIX C: RATE EQUATIONS FOR THE TEMPERATURE MODEL}

In this Appendix, we present the derivation of the hot carrier recombination and generation rate by the optical phonon emission and absorption process, respectively, used in the temperature model. The Hamiltonian of electron-phonon interaction $H_{e p}$ is

$$
H_{e p}=\sum_{\boldsymbol{k}, \boldsymbol{k}^{\prime}, \boldsymbol{q}} V_{e p}\left(c_{\boldsymbol{k}}^{\dagger} c_{\boldsymbol{k}^{\prime}} b_{\boldsymbol{q}}+c_{\boldsymbol{k}^{\prime}}^{\dagger} c_{\boldsymbol{k}} b_{-\boldsymbol{q}}^{\dagger}\right) .
$$

Here, $V_{e p}$ is the potential of the electron-phonon interaction, $c_{k}^{\dagger}$ $\left(c_{k}\right)$ is the creation (annihilation) operator with carrier wave vector $\boldsymbol{k}$, and $b_{\boldsymbol{q}}^{\dagger}\left(b_{\boldsymbol{q}}\right)$ is the creation (annihilation) operator with phonon wave vector $\boldsymbol{q}$. From Fermi's golden rule, the carrier transition rate from $\boldsymbol{k}$ to $\boldsymbol{k}^{\prime}$ by the emission and absorption of the $\boldsymbol{\Gamma}_{\mathrm{LO}}$ phonon or $\boldsymbol{\Gamma}_{\mathrm{TO}}$ phonon with an energy of $\hbar \omega_{\boldsymbol{\Gamma}}$ is given by

$$
\begin{aligned}
P_{\lambda \boldsymbol{k} \lambda^{\prime} \boldsymbol{k}^{\prime}}^{\mathrm{EM} / \mathrm{AB}, \boldsymbol{\Gamma}}= & \frac{2 \pi}{\hbar}\left|\left\langle\boldsymbol{k}^{\prime}, \lambda^{\prime}\left|H_{e p}\right| \boldsymbol{k}, \lambda\right\rangle\right|^{2} \delta\left(\varepsilon_{\lambda^{\prime} \boldsymbol{k}^{\prime}}-\varepsilon_{\lambda \boldsymbol{k}} \pm \hbar \omega_{\boldsymbol{\Gamma}}\right) \\
= & \frac{\pi\left|\mathrm{D}_{\lambda \boldsymbol{k} \lambda^{\prime} \boldsymbol{k}^{\prime}}\right|^{2}}{\rho \omega_{\boldsymbol{\Gamma}} A}\left(n_{\boldsymbol{\Gamma}}+\frac{1}{2} \pm \frac{1}{2}\right) \\
& \times \delta\left(\varepsilon_{\lambda^{\prime} \boldsymbol{k}^{\prime}}-\varepsilon_{\lambda \boldsymbol{k}} \pm \hbar \omega_{\boldsymbol{\Gamma}}\right) \delta\left(\boldsymbol{k}^{\prime}-\boldsymbol{k} \pm \boldsymbol{q}\right),
\end{aligned}
$$

$$
\begin{aligned}
P_{\lambda \boldsymbol{k} \lambda^{\prime} \boldsymbol{k}^{\prime}}^{\mathrm{EM} / \mathrm{AB}, \mathbf{K}}= & \frac{2 \pi}{\hbar}\left|\left\langle\boldsymbol{k}^{\prime}, \lambda^{\prime}\left|H_{e p}\right| \boldsymbol{k}, \lambda\right\rangle\right|^{2} \delta\left(\varepsilon_{\lambda^{\prime} \boldsymbol{k}^{\prime}}-\varepsilon_{\lambda \boldsymbol{k}} \pm \hbar \omega_{\boldsymbol{\Gamma}}\right) \\
= & \frac{\pi\left|\mathrm{D}_{\lambda \boldsymbol{k} \lambda^{\prime} \boldsymbol{k}^{\prime}}^{\mathbf{K}}\right|^{2}}{\rho \omega_{\boldsymbol{\Gamma}} A}\left(n_{\mathbf{K}}+\frac{1}{2} \pm \frac{1}{2}\right) \\
& \times \delta\left(\varepsilon_{\lambda^{\prime} \boldsymbol{k}^{\prime}}-\varepsilon_{\lambda \boldsymbol{k}} \pm \hbar \omega_{\boldsymbol{\Gamma}}\right) \delta\left(\boldsymbol{k}^{\prime}-\boldsymbol{k} \pm \boldsymbol{q}\right) .
\end{aligned}
$$

Here, $\left|\mathrm{D}_{\lambda \boldsymbol{k} \lambda^{\prime} \boldsymbol{k}^{\prime}}^{\Gamma}\right|^{2}$ and $\left|\mathrm{D}_{\lambda \boldsymbol{k} \lambda^{\prime} \boldsymbol{k}^{\prime}}^{\mathbf{K}}\right|^{2}$ are the squares of the EPC matrix elements. For small $\boldsymbol{q}$ and $\boldsymbol{k}$, the EPC matrix elements are given by Eq. (7). $\rho$ is the mass density, $A$ is the area of the graphene sample, $\varepsilon_{\lambda \boldsymbol{k}}=\lambda \hbar v_{F}|\boldsymbol{k}|$ is the energy of the 2D MDF, and $\lambda= \pm 1$ is the band index. The upper and lower signs correspond to the optical phonon emission and absorption process, respectively. The corresponding hot carrier recombination and generation rates per unit area including both intraand interband transitions are written as

$$
\begin{aligned}
R_{\boldsymbol{\Gamma}}= & \frac{1}{A} \sum_{\lambda, \lambda^{\prime}} \sum_{\boldsymbol{k}} \sum_{\boldsymbol{k}^{\prime}} P_{\lambda \boldsymbol{k} \lambda^{\prime} \boldsymbol{k}^{\prime}}^{\mathrm{EM}, \boldsymbol{\Gamma}} f_{\lambda}(\boldsymbol{k})\left[1-f_{\lambda^{\prime}}\left(\boldsymbol{k}^{\prime}\right)\right] \\
= & \frac{1}{A} \sum_{\lambda, \lambda^{\prime}} \sum_{\boldsymbol{k}} \sum_{\boldsymbol{k}^{\prime}} \frac{\pi\left\langle\mathrm{D}_{\boldsymbol{\Gamma}}^{2}\right\rangle_{F}\left[1 \pm \cos \left(\theta_{\boldsymbol{k}, \boldsymbol{q}}+\theta_{\boldsymbol{k}^{\prime}, \boldsymbol{q}}\right)\right]}{\rho \omega_{\boldsymbol{\Gamma}} A}\left(n_{\boldsymbol{\Gamma}}+1\right) \\
& \times f_{\lambda}(\boldsymbol{k})\left[1-f_{\lambda^{\prime}}\left(\boldsymbol{k}^{\prime}\right)\right] \delta\left(\varepsilon_{\lambda^{\prime} \boldsymbol{k}^{\prime}}-\varepsilon_{\lambda \boldsymbol{k}}+\hbar \omega_{\boldsymbol{\Gamma}}\right) \delta\left(\boldsymbol{k}^{\prime}-\boldsymbol{k}+\boldsymbol{q}\right) \\
= & \sum_{\lambda, \lambda^{\prime}, \boldsymbol{k}} \frac{\pi\left\langle\mathrm{D}_{\boldsymbol{\Gamma}}^{2}\right\rangle_{F}}{A(2 \pi)^{2}} \int d^{2} \boldsymbol{k}^{\prime} \frac{\left[1 \pm \cos \left(\theta_{\boldsymbol{k}, \boldsymbol{q}}+\theta_{\boldsymbol{k}^{\prime}, \boldsymbol{q}}\right)\right]}{\rho \omega_{\boldsymbol{\Gamma}_{\mathrm{LO}}}}\left(n_{\boldsymbol{\Gamma}}+1\right) \\
& \times f_{\lambda}(\boldsymbol{k})\left[1-f_{\lambda^{\prime}}\left(\boldsymbol{k}^{\prime}\right)\right] \delta\left(\varepsilon_{\lambda^{\prime} \boldsymbol{k}^{\prime}}-\varepsilon_{\lambda \boldsymbol{k}}+\hbar \omega_{\boldsymbol{\Gamma}}\right) \delta\left(\boldsymbol{k}^{\prime}-\boldsymbol{k}+\boldsymbol{q}\right) \\
= & \sum_{\lambda, \lambda^{\prime}} \frac{\left\langle\mathrm{D}_{\boldsymbol{\Gamma}}^{2}\right\rangle_{F}\left(n_{\boldsymbol{\Gamma}}+1\right)}{4 \pi \rho \omega_{\boldsymbol{\Gamma}}} \int f_{\lambda}\left(\varepsilon_{\lambda \boldsymbol{k}}\right) N\left(\varepsilon_{\lambda \boldsymbol{k}}\right) d \varepsilon_{\lambda \boldsymbol{k}} \\
& \times \int d^{2} \boldsymbol{k}^{\prime}\left[1 \pm \cos \left(\theta_{\boldsymbol{k}, \boldsymbol{q}}+\theta_{\boldsymbol{k}^{\prime}, \boldsymbol{q}}\right)\right]\left[1-f_{\lambda^{\prime}}\left(\boldsymbol{k}^{\prime}\right)\right] \\
& \times \delta\left(\varepsilon_{\lambda^{\prime} \boldsymbol{k}^{\prime}}-\varepsilon_{\lambda \boldsymbol{k}}+\hbar \omega_{\boldsymbol{\Gamma}}\right) \delta\left(\boldsymbol{k}^{\prime}-\boldsymbol{k}+\boldsymbol{q}\right),
\end{aligned}
$$




$$
\begin{aligned}
G_{\boldsymbol{\Gamma}}= & \frac{1}{A} \sum_{\lambda, \lambda^{\prime}} \sum_{\boldsymbol{k}} \sum_{\boldsymbol{k}^{\prime}} P_{\lambda \boldsymbol{k} \lambda^{\prime} \boldsymbol{k}^{\prime}}^{\mathrm{AB}, \boldsymbol{\Gamma}} f_{\lambda}(\boldsymbol{k})\left[1-f_{\lambda^{\prime}}\left(\boldsymbol{k}^{\prime}\right)\right] \\
= & \frac{1}{A} \sum_{\lambda, \lambda^{\prime}} \sum_{\boldsymbol{k}} \sum_{\boldsymbol{k}^{\prime}} \frac{\pi\left\langle\mathrm{D}_{\boldsymbol{\Gamma}}^{2}\right\rangle_{F}\left[1 \pm \cos \left(\theta_{\boldsymbol{k}, \boldsymbol{q}}+\theta_{\boldsymbol{k}^{\prime}, \boldsymbol{q}}\right)\right]}{\rho \omega_{\boldsymbol{\Gamma}} A} n_{\boldsymbol{\Gamma}} \\
& \times f_{\lambda}(\boldsymbol{k})\left[1-f_{\lambda^{\prime}}\left(\boldsymbol{k}^{\prime}\right)\right] \delta\left(\varepsilon_{\lambda^{\prime} \boldsymbol{k}^{\prime}}-\varepsilon_{\lambda \boldsymbol{k}}-\hbar \omega_{\boldsymbol{\Gamma}}\right) \delta\left(\boldsymbol{k}^{\prime}-\boldsymbol{k}-\boldsymbol{q}\right) \\
= & \sum_{\lambda, \lambda^{\prime}} \sum_{\boldsymbol{k}} \frac{\pi\left\langle\mathrm{D}_{\boldsymbol{\Gamma}}^{2}\right\rangle_{F}}{A(2 \pi)^{2}} \int d^{2} \boldsymbol{k}^{\prime} \frac{\left[1 \pm \cos \left(\theta_{\boldsymbol{k}, \boldsymbol{q}}+\theta_{\boldsymbol{k}^{\prime}, \boldsymbol{q}}\right)\right]}{\rho \omega_{\boldsymbol{L}_{\mathrm{LO}}}} n_{\boldsymbol{\Gamma}} \\
& \times f_{\lambda}(\boldsymbol{k})\left[1-f_{\lambda^{\prime}}\left(\boldsymbol{k}^{\prime}\right)\right] \delta\left(\varepsilon_{\lambda^{\prime} \boldsymbol{k}^{\prime}}-\varepsilon_{\lambda \boldsymbol{k}}-\hbar \omega_{\boldsymbol{\Gamma}}\right) \delta\left(\boldsymbol{k}^{\prime}-\boldsymbol{k}-\boldsymbol{q}\right) \\
= & \sum_{\lambda, \lambda^{\prime}} \frac{\left\langle\mathrm{D}_{\boldsymbol{\Gamma}}^{2}\right\rangle_{F} n_{\boldsymbol{\Gamma}}}{4 \pi \rho \omega_{\boldsymbol{\Gamma}}} \int f_{\lambda}\left(\varepsilon_{\lambda \boldsymbol{k}}\right) N\left(\varepsilon_{\lambda \boldsymbol{k}}\right) d \varepsilon_{\lambda \boldsymbol{k}} \\
& \times \int d^{2} \boldsymbol{k}^{\prime}\left[1 \pm \cos \left(\theta_{\boldsymbol{k}, \boldsymbol{q}}+\theta_{\boldsymbol{k}^{\prime}, \boldsymbol{q}}\right)\right]\left[1-f_{\lambda^{\prime}}\left(\boldsymbol{k}^{\prime}\right)\right] \\
& \times \delta\left(\varepsilon_{\lambda^{\prime} \boldsymbol{k}^{\prime}}-\varepsilon_{\lambda \boldsymbol{k}}-\hbar \omega_{\boldsymbol{\Gamma}}\right) \delta\left(\boldsymbol{k}^{\prime}-\boldsymbol{k}-\boldsymbol{q}\right) .
\end{aligned}
$$

Here, $N\left(\varepsilon_{\lambda k}\right)=2\left|\varepsilon_{\lambda k}\right| / \pi\left(\hbar v_{F}\right)^{2}$ is the density of states of the 2D MDF. Furthermore, the electron distribution function $f_{\lambda}(\boldsymbol{k})$ can be replaced by Fermi-Dirac-type distribution $f_{0}\left(\varepsilon_{\lambda k}, T_{e}\right)$ for hot carriers in quasiequilibrium. Similarly, the hot carrier recombination and generation rates by the $\mathbf{K}$ phonon with an energy of $\hbar \omega_{\mathbf{K}}$ are given by

$$
\begin{aligned}
R_{\mathbf{K}}= & \frac{1}{A} \sum_{\lambda, \lambda^{\prime}} \sum_{\boldsymbol{k}} \sum_{\boldsymbol{k}^{\prime}} P_{\lambda \boldsymbol{k} \lambda^{\prime} \boldsymbol{k}^{\prime}}^{\mathrm{EM}, \mathbf{K}} f_{\lambda}(\boldsymbol{k})\left[1-f_{\lambda^{\prime}}\left(\boldsymbol{k}^{\prime}\right)\right] \\
= & \sum_{\lambda, \lambda^{\prime}} \frac{\left\langle\mathrm{D}_{\mathbf{K}}^{2}\right\rangle_{F}\left(n_{\mathbf{K}}+1\right)}{4 \pi \rho \omega_{\mathbf{K}}} \int f_{\lambda}\left(\varepsilon_{\lambda \boldsymbol{k}}\right) N\left(\varepsilon_{\lambda \boldsymbol{k}}\right) d \varepsilon_{\lambda \boldsymbol{k}} \\
& \times \int d^{2} \boldsymbol{k}^{\prime}\left[1 \pm \cos \left(\theta_{\boldsymbol{k}, \boldsymbol{k}^{\prime}}\right)\right]\left[1-f_{\lambda^{\prime}}\left(\boldsymbol{k}^{\prime}\right)\right] \\
& \times \delta\left(\varepsilon_{\lambda^{\prime} \boldsymbol{k}^{\prime}}-\varepsilon_{\lambda \boldsymbol{k}}+\hbar \omega_{\mathbf{K}}\right) \delta\left(\boldsymbol{k}^{\prime}-\boldsymbol{k}+\boldsymbol{q}\right), \\
G_{\mathbf{K}}= & \frac{1}{A} \sum_{\lambda, \lambda^{\prime}} \sum_{\boldsymbol{k}} \sum_{\boldsymbol{k}^{\prime}} P_{\lambda \boldsymbol{k}, \boldsymbol{k}^{\prime} \boldsymbol{k}^{\prime}}^{\mathrm{AB}} f_{\lambda}(\boldsymbol{k})\left[1-f_{\lambda^{\prime}}\left(\boldsymbol{k}^{\prime}\right)\right] \\
= & \sum_{\lambda, \lambda^{\prime}} \frac{\left\langle\mathrm{D}_{\mathbf{K}}^{2}\right\rangle_{F} n_{\mathbf{K}}}{4 \pi \rho \omega_{\mathbf{K}}} \int f_{\lambda}\left(\varepsilon_{\lambda \boldsymbol{k}}\right) N\left(\varepsilon_{\lambda \boldsymbol{k}}\right) d \varepsilon_{\lambda \boldsymbol{k}} \\
& \times \int d^{2} \boldsymbol{k}^{\prime}\left[1 \pm \cos \left(\theta_{\boldsymbol{k}, \boldsymbol{k}^{\prime}}\right)\right]\left[1-f_{\lambda^{\prime}}\left(\boldsymbol{k}^{\prime}\right)\right] \\
& \times \delta\left(\varepsilon_{\lambda^{\prime} \boldsymbol{k}^{\prime}}-\varepsilon_{\lambda \boldsymbol{k}}-\hbar \omega_{\mathbf{K}}\right) \delta\left(\boldsymbol{k}^{\prime}-\boldsymbol{k}-\boldsymbol{q}\right) .
\end{aligned}
$$

Using Eqs. (C3a), (C3b), (C4a), and (C4b), the total balance between the optical phonon emission and absorption rate is given by $R_{\eta}^{\mathrm{Net}}=R_{\eta}-G_{\eta}$.

In Eqs. (9a) and (9b), $R_{M, \eta}^{\mathrm{Net}}=R_{\eta}-G_{\eta}$ denotes the total balance between the optical phonon emission and absorption rate per number of phonon modes.

$$
\begin{aligned}
R_{M, \eta} & =\frac{1}{A} \sum_{\lambda, \lambda^{\prime}} \sum_{\boldsymbol{k}, \boldsymbol{k}^{\prime}} P_{\lambda \boldsymbol{k} \lambda^{\prime} \boldsymbol{k}^{\prime}}^{\mathrm{EM}, \eta} f_{\lambda}(\boldsymbol{k})\left[1-f_{\lambda^{\prime}}\left(\boldsymbol{k}^{\prime}\right)\right] / M_{\eta}^{-}(\lambda \boldsymbol{k}), \\
G_{M, \eta} & =\frac{1}{A} \sum_{\lambda, \lambda^{\prime}} \sum_{\boldsymbol{k}, \boldsymbol{k}^{\prime}} P_{\lambda \boldsymbol{k} \lambda^{\prime} \boldsymbol{k}^{\prime}}^{\mathrm{AB}, \eta} f_{\lambda}(\boldsymbol{k})\left[1-f_{\lambda^{\prime}}\left(\boldsymbol{k}^{\prime}\right)\right] / M_{\eta}^{+}(\lambda \boldsymbol{k}) .
\end{aligned}
$$

Here, $M_{\eta}^{-}(\lambda \boldsymbol{k})$ and $M_{\eta}^{+}(\lambda \boldsymbol{k})$ are the number of $\eta$-phonon modes $(\boldsymbol{q})$ per unit area that participate in the phonon emission and absorption processes for carrier state $(\lambda, \boldsymbol{k})$, respectively.

$$
\begin{aligned}
M_{\eta}^{ \pm}(\lambda \boldsymbol{k}) & =\frac{a_{\eta}}{A}\left(\pi|\boldsymbol{q}|_{\max }^{2}-\pi|\boldsymbol{q}|_{\min }^{2}\right) /|\Delta \boldsymbol{q}|^{2} \\
& =\frac{a_{\eta} \pi}{A}\left|\left(\frac{2 \varepsilon_{\lambda \boldsymbol{k}} \pm \hbar \omega_{\eta}}{\hbar v_{F}}\right)^{2}-\left(\frac{\hbar \omega_{\eta}}{\hbar v_{F}}\right)^{2}\right| /\left(\frac{4 \pi^{2}}{A}\right) \\
& =\frac{a_{\eta}}{4 \pi}\left|\left\{\left(\frac{2 \varepsilon_{\lambda \boldsymbol{k}} \pm \hbar \omega_{\eta}}{\hbar v_{F}}\right)^{2}-\left(\frac{\omega_{\eta}}{v_{F}}\right)^{2}\right\}\right| .
\end{aligned}
$$

In this case, $a_{\Gamma}=1$ for $\boldsymbol{\Gamma}_{\mathrm{LO}}$ and $\boldsymbol{\Gamma}_{\mathrm{TO}}$ phonons, and $a_{\mathrm{K}}=2$ for $\mathbf{K}$ phonons. The factor of $a_{\mathbf{K}}=2$ represents the degenerate phonon valleys at the $\mathbf{K}$ and $\mathbf{K}^{\prime}$ points. Using Eqs. (C5a) and (C5b), the total balance between the optical phonon emission and absorption rate per number of phonon modes is given by $R_{M, \eta}^{\mathrm{Net}}=R_{M, \eta}-G_{M, \eta}$.

\section{APPENDIX D: PUMP POWER INJECTED INTO THE GRAPHENE SAMPLE CONSIDERING SATURABLE ABSORPTION}

In this Appendix, we present the derivation of the pump intensity $\mathcal{F}_{\text {ab }}$ injected into the graphene sample, considering the multiple reflections inside the substrate and the saturable absorption (SA) effect. The SA is an extreme nonlinear phenomenon that consists of the quenching of the optical absorption under high-intensity illumination. Following Marini et al. [111], we introduce the derivation of saturable absorption coefficient $\alpha_{\text {inter }}$ in graphene. Thereafter, we explain the derivation of the absorbed pump intensity $\mathcal{F}_{\mathrm{ab}}$ by graphene on the substrate at an oblique incidence angle using $\alpha_{\text {inter }}$.

We study the response of a single electron in graphene under an in-plane $x$-direction applied field $\mathbf{E}(t)=E_{0} e^{-i \omega t} \hat{\mathbf{x}}$. The extended Bloch equations describing the temporal variation in the interband coherence $\rho_{\mathbf{k}}$ and population difference $n_{\mathbf{k}}$ in photoexcited graphene are as follows:

$$
\begin{aligned}
& \dot{\rho}_{\mathbf{k}}(t)=-\frac{i}{2} \dot{\theta}_{\mathbf{k}}(t) n_{\mathbf{k}}(t) e^{2 i \Omega_{\mathbf{k}}(t)}-\frac{\rho_{\mathbf{k}}(t)}{\tau_{\mathrm{ie}}}, \\
& \dot{n}_{\mathbf{k}}(t)=2 \dot{\theta}_{\mathbf{k}}(t) \operatorname{Im}\left\{\rho_{\mathbf{k}}(t) e^{-2 i \Omega_{\mathbf{k}}(t)}\right\}-\frac{n_{\mathbf{k}}(t)}{\tau_{\mathrm{ie}}},
\end{aligned}
$$

where $\pi=\hbar \mathbf{k}=\hbar k(\cos \phi, \sin \phi)$ is the electron momentum, the global dynamical phase $\Omega_{\mathbf{k}}(t)$ is defined as $\Omega_{\mathbf{k}}(t)=v_{\mathrm{F}} \int|\mathbf{k}+(e / \hbar c) \mathbf{A}(t)| d t$, and $\theta_{\mathbf{k}}(t)=\operatorname{atan}\left\{k_{y} /\left[k_{x}+\right.\right.$ $(e / \hbar c) A(t)]\}$ is the time-dependent directional angle of the electron quasimomentum $\pi(t)=\hbar \mathbf{k}+(e / c) \mathbf{A}(t)$. The vector potential $\mathbf{A}(t)$ is $\mathbf{A}(t)=-\int \mathbf{E}(t) d t$. In the near-resonant condition, the optical momentum is negligible [i.e., $\hbar k \gg$ $(e / c) A(t)]$ and does not significantly affect the interband dynamics. In this approximation, Eqs. (D1a) and (D1b) are reduced to

$$
\begin{aligned}
& \dot{\Gamma}_{\mathbf{k}}=-\left(\frac{1}{\tau_{\mathrm{ie}}}+2 i \omega_{0}\right) \Gamma_{\mathbf{k}}-\frac{i e}{\hbar k} \operatorname{Re}\left\{E_{0} e^{-i \omega t}\right\} \sin \phi n_{\mathrm{k}}, \\
& \dot{n}_{\mathrm{k}}=-\frac{1}{\tau_{\mathrm{ie}}}\left[n_{\mathbf{k}}-\mathcal{N}\right]+\frac{4 e}{\hbar k} \operatorname{Re}\left\{E_{0} e^{-i \omega t}\right\} \sin \phi \operatorname{Im}\left\{\Gamma_{\mathbf{k}}\right\} .
\end{aligned}
$$


In this case, $\Gamma_{\mathbf{k}}(t)=\rho_{\mathbf{k}}(t) e^{-2 i \omega_{0} t}, \omega_{0}=v_{\mathrm{F}}|\mathbf{k}|, \mathcal{N}\left(k, T_{e}\right)=$ $\mathcal{F}\left(\mathbf{k}, T_{e}\right)-\mathcal{F}\left(-\mathbf{k}, T_{e}\right), \quad$ where $\quad \mathcal{F}\left(\mathbf{k}, T_{e}\right)=1 /\{1+$ $\left.\exp \left[\left(v_{F} \hbar|\mathbf{k}|-\mu\right) / k_{B} T_{e}\right]\right\}$. A phenomenological relaxation time $\tau_{\mathrm{ie}}$ is introduced, which encompasses the effect of numerous ultrafast decay channels for the out-of-equilibrium electrons into hot carriers and phonons. The steady-state ansatz for the Bloch equation is given by

$$
\begin{aligned}
& \Gamma_{\mathbf{k}}(t)=\Gamma_{\mathbf{k}}^{+} e^{i \omega t}+\Gamma_{\mathbf{k}}^{-} e^{-i \omega t}, \\
& n_{\mathbf{k}}(t)=n_{\mathbf{k}}^{(0)}+\operatorname{Re}\left\{n_{\mathbf{k}}^{(2)} e^{-2 i \omega t}\right\} .
\end{aligned}
$$

Using these expressions and neglecting the higher-harmonic terms, Eqs. (D2a) and (D2b) lead to

$$
n_{\mathbf{k}}^{(0)}=\mathcal{N}+4 \xi \operatorname{Im}\left\{\frac{1-i \omega \tau_{\mathrm{ie}}}{1-i \omega+\tau_{\mathrm{ie}}} \Gamma_{\mathbf{k}}^{-}\right\}
$$

$$
\begin{aligned}
& n_{\mathbf{k}}^{(2)}=\frac{-4 i \xi\left(1-i \omega \tau_{\mathrm{ie}}\right) \Gamma_{\mathbf{k}}^{-}}{\left(1-2 i \omega \tau_{\mathrm{ie}}\right)\left(1-i \omega_{+} \tau_{\mathrm{ie}}\right)}, \\
& \Gamma_{\mathbf{k}}^{+}=-\frac{1+i \omega_{-} \tau_{\mathrm{ie}}}{1+i \omega_{+} \tau_{\mathrm{ie}}} \Gamma_{\mathbf{k}}^{-*}, \\
& \Gamma_{\mathbf{k}}^{-}=\frac{(-i \xi / 2)}{1-i \omega_{-} \tau_{\mathrm{ie}}}\left(n_{\mathbf{k}}^{(0)}+\frac{1}{2} n_{\mathbf{k}}^{(2)}\right),
\end{aligned}
$$

where $\xi=\left(e \tau_{\mathrm{ie}} E_{0} / \hbar k\right) \sin \phi$ and $\omega_{ \pm}=\omega \pm 2 \omega_{0}$. The macroscopic interband current density depending on the light intensity $I_{0}=(c / 2 \pi)\left|E_{0}\right|^{2}$ at the electronic temperature $T_{e}$ is determined by

$$
\mathbf{J}_{\text {inter }}(t)=-\frac{2 e v_{\mathrm{F}}}{\pi^{2}} \operatorname{Re}\left\{i e^{-i \omega t} \int \sin \phi\left[\Gamma_{\mathbf{k}}^{-}-\Gamma_{\mathbf{k}}^{+*}\right] d^{2} \mathbf{k}\right\} \hat{\mathbf{x}},
$$

where $\Gamma_{\mathbf{k}}^{-}-\Gamma_{\mathbf{k}}^{+*}=-\frac{i e \tau_{\mathrm{i}} E_{0} \sin \phi\left(1-i \omega \tau_{\mathrm{i}}\right)}{2 \hbar k\left(1-i \omega_{+} \tau_{\mathrm{ie}}\right)\left(1-i \omega_{-} \tau_{\mathrm{ie}}\right)}\left[2 n_{\mathbf{k}}^{(0)}+n_{\mathbf{k}}^{(2)}\right]$, and

$$
n_{\mathbf{k}}^{(0)}=\frac{\mathcal{N}}{1+2 \xi^{2} \operatorname{Im}\left\{\frac{i\left(1-i \omega \tau_{\mathrm{ie}}\right)}{\left(1-i \omega_{+} \tau_{\mathrm{ie}}\right)\left(1-i \omega-\tau_{\mathrm{ie}}\right)}\left[1-\frac{\xi^{2}\left(1-i \omega \tau_{\mathrm{ie}}\right)}{\left(1-2 i \omega \tau_{\mathrm{ie}}\right)\left(1-i \omega_{+} \tau_{\mathrm{ie}}\right)\left(1-i \omega-\tau_{\mathrm{ie}}\right)+\xi^{2}\left(1-i \omega \tau_{\mathrm{ie}}\right)}\right]\right\}}
$$

and

$$
n_{\mathbf{k}}^{(2)}=\frac{-2 \xi^{2}\left(1-i \omega \tau_{\mathrm{ie}}\right) \mathcal{N} /\left[\left(1-2 i \omega \tau_{\mathrm{ie}}\right)\left(1-i \omega_{+} \tau_{\mathrm{ie}}\right)\left(1-i \omega_{-} \tau_{\mathrm{ie}}\right)+\xi^{2}\left(1-i \omega \tau_{\mathrm{ie}}\right)\right]}{1+2 \xi^{2} \operatorname{Im}\left\{\frac{i\left(1-i \omega \tau_{\mathrm{ie}}\right)}{\left(1-i \omega_{+} \tau_{\mathrm{ie}}\right)\left(1-i \omega_{-} \tau_{\mathrm{ie}}\right)}\left[1-\frac{\xi^{2}\left(1-i \omega \tau_{\mathrm{ie}}\right)}{\left(1-2 i \omega \tau_{\mathrm{ie}}\right)\left(1-i \omega_{+}+\tau_{\mathrm{ie}}\right)\left(1-i \omega_{-} \tau_{\mathrm{ie}}\right)+\xi^{2}(1-i \omega \tau)}\right]\right\}} .
$$

Subsequently, by expressing the integral over the reciprocal space in polar coordinates, the following is obtained:

$$
\mathbf{J}_{\text {inter }}(t)=-\frac{8 e^{2} v_{\mathrm{F}} \tau_{\text {ie }}}{\pi^{2} \hbar} \operatorname{Re}\left\{E_{0} e^{-i \omega t}\left(1-i \omega \tau_{\text {ie }}\right) \int_{0}^{\pi / 2} d \phi \int_{0}^{\infty} d k \frac{\sin ^{2} \phi\left[2 n_{\mathrm{k}}^{(0)}+n_{\mathrm{k}}^{(2)}\right]}{2\left(1-i \omega_{+} \tau_{\text {ie }}\right)\left(1-i \omega_{-} \tau_{\text {ie }}\right)}\right\} \hat{\mathbf{x}} .
$$

Using the interband current, the interband absorption coefficient is determined as the ratio of the time-averaged absorbed power over an optical cycle to the incident intensity $I_{0}$ :

$$
\alpha_{\text {inter }}\left(I_{0}\right) \equiv \frac{\int_{-\pi / \omega}^{+\pi / \omega} \mathbf{J}_{\text {inter }}(t) \cdot \mathbf{E}(t) d t}{(2 \pi / \omega) I_{0}} .
$$

Although the above results were obtained under continuous-wave (CW) illumination conditions, these are also applicable to commonly used optical pulses that have a large duration compared with the optical period.

Taking into account the SA for the interband transition by the pump irradiation, the transmission and reflection coefficients of the s-polarized pump pulse incident on the system of layer $i /$ graphene/layer $j$ from layer $i$, as illustrated in Fig. 10, are calculated by

$$
\begin{aligned}
t_{\mathrm{ij}}^{s}\left(I_{0}, \gamma_{\mathrm{ij}}\right) & =\frac{2 \epsilon_{i}^{1 / 2}\left(\omega_{\text {pump }}\right) \cos \theta_{\mathrm{i}}}{\alpha_{\text {inter }}\left(\gamma_{\mathrm{ij}} I_{0}\right)+\left[\epsilon_{\mathrm{j}}\left(\omega_{\text {pump }}\right)-\epsilon_{\mathrm{i}}\left(\omega_{\text {pump }}\right) \sin ^{2} \theta_{\mathrm{i}}\right]^{1 / 2}+\epsilon_{\mathrm{i}}\left(\omega_{\text {pump }}\right)^{1 / 2} \cos \theta_{\mathrm{i}}}, \\
r_{\mathrm{ij}}^{s}\left(I_{0}, \gamma_{\mathrm{ij}}\right) & =-\frac{\alpha_{\text {inter }}\left(\omega, \gamma_{\mathrm{ij}} I_{0}, T_{e}\right)+\left[\epsilon_{\mathrm{j}}\left(\omega_{\mathrm{p}}\right)-\epsilon_{\mathrm{i}}\left(\omega_{\mathrm{p}}\right) \sin ^{2} \theta_{\mathrm{i}}\right]^{1 / 2}-\epsilon_{\mathrm{i}}^{1 / 2}\left(\omega_{\mathrm{p}}\right) \cos \theta_{\mathrm{i}}}{\alpha_{\text {inter }}\left(\omega, \gamma_{\mathrm{ij}} I_{0}, T_{e}\right)+\left[\epsilon_{\mathrm{j}}\left(\omega_{\mathrm{p}}\right)-\epsilon_{\mathrm{i}}\left(\omega_{\mathrm{p}}\right) \sin ^{2} \theta_{\mathrm{i}}\right]^{1 / 2}+\epsilon_{\mathrm{i}}^{1 / 2}\left(\omega_{\mathrm{p}}\right) \cos \theta_{\mathrm{i}}} .
\end{aligned}
$$

In this case, the pump pulse irradiates the graphene from layer $i$ with the incidence angle of $\theta_{\mathrm{i}}$ and transmits it to layer $j$ with the angle $\theta_{\mathrm{j}}$. Moreover, $\gamma_{\mathrm{ij}}$ is the correction factor. Although $\alpha_{\text {inter }}\left(I_{0}\right)$ is appropriate for the case in which the optical pump pulse excites the suspended graphene at the normal incidence angle, the saturation behavior will change when graphene on a substrate is excited by a pump pulse at an oblique incidence angle, where the injected pump power becomes smaller by a factor of $\gamma_{\mathrm{ij}}$. The corresponding transmittance and reflectance are determined by

$$
\begin{aligned}
& T_{\mathrm{ij}}^{s}\left(I_{0}, \gamma_{\mathrm{ij}}\right)=\left|t_{\mathrm{ij}}^{s}\left(I_{0}, \gamma_{\mathrm{ij}}\right)\right|^{2} \frac{\epsilon_{\mathrm{j}}^{1 / 2}\left(\omega_{\mathrm{p}}\right) \cos \theta_{\mathrm{j}}}{\epsilon_{\mathrm{i}}^{1 / 2}\left(\omega_{\mathrm{p}}\right) \cos \theta_{\mathrm{i}}}, \\
& R_{\mathrm{ij}}^{s}\left(I_{0}, \gamma_{\mathrm{ij}}\right)=\left|r_{\mathrm{ij}}^{s}\left(I_{0}, \gamma_{\mathrm{ij}}\right)\right|^{2} .
\end{aligned}
$$

Using Eqs. (D10a) and (D10b), the absorption of the pump pulse by the graphene layer is provided by

$$
A_{\mathrm{ij}}^{s}\left(I_{0}, \gamma_{\mathrm{ij}}\right)=1-T_{\mathrm{ij}}^{s}\left(I_{0}, \gamma_{\mathrm{ij}}\right)-R_{\mathrm{ij}}^{s}\left(I_{0}, \gamma_{\mathrm{ij}}\right) .
$$


The correction factor $\gamma_{\mathrm{ij}}$ is calculated by the ratio of the absorption coefficient

$$
\gamma_{\mathrm{ij}}=\frac{A_{\mathrm{ij}}^{S}\left(I_{0}, \gamma_{\mathrm{ij}}\right)}{\alpha_{\text {inter }}\left(I_{0}\right)}
$$

and can be determined self-consistently. Using the converged $\gamma_{\mathrm{ij}}^{*}$, the transmittance, reflectance, and absorption coefficients in the experimental condition are obtained by

$$
\begin{aligned}
& T_{\mathrm{ij}}^{s *}\left(I_{0}\right)=T_{\mathrm{ij}}^{s}\left(I_{0}, \gamma_{\mathrm{ij}}^{*}\right), \\
& R_{\mathrm{ij}}^{s *}\left(I_{0}\right)=R_{\mathrm{ij}}^{s}\left(I_{0}, \gamma_{\mathrm{ij}}^{*}\right), \\
& A_{\mathrm{ij}}^{s *}\left(I_{0}\right)=A_{\mathrm{ij}}^{s}\left(I_{0}, \gamma_{\mathrm{ij}}^{*}\right) .
\end{aligned}
$$

The envelope function of the pump pulse considering the $n$th multiple reflections inside the substrate is given by

$$
\begin{aligned}
\mathcal{I}(t) & =\sum_{n=0} \mathcal{I}_{n}(t+n \Delta T) \\
& =\mathcal{I}_{0}(t)+T_{12}^{s *} R_{23}^{s} \sum_{n=1} \mathcal{I}_{0}(t+n \Delta T)\left(R_{21}^{s *} R_{23}^{s}\right)^{n-1} .
\end{aligned}
$$

In this case, $\mathcal{I}_{0}(t)$ represents the incident pump pulse, which is assumed to have hyperbolic secant form $\mathcal{I}_{0}(t)=$ $\left(F_{0} / 2 \tau_{\text {pump }}\right) \operatorname{sech}^{2}\left(t / \tau_{\text {pump }}\right)$, where $F_{0}$ is the fluence and $2 \tau_{\text {pump }}$ is the pulse duration. $\mathcal{I}_{n}(t)=\left(F_{\mathrm{n}} / 2 \tau_{\text {pump }}\right) \operatorname{sech}^{2}\left(t / \tau_{\text {punmp }}\right)$ represents the $n$th reflection of the incident pump pulse and $F_{\mathrm{n}}$ is the fluence of the $n$th reflection pulse. $\Delta T$ is the time delay owing to one round trip in the substrate, respectively. Using Eq. (D15) and $I_{0}=F_{0} / 2 \tau_{\text {pump }}, F_{n}$ for $n \geqslant 1$ is obtained by

$$
\begin{aligned}
& F_{1}=F_{0} T_{12}^{s *}\left(F_{0} / 2 \tau_{\text {pump }}\right) R_{23}^{s}, \\
& F_{n}=F_{n-1} R_{21}^{s *}\left(F_{\mathrm{n}-1}\right) R_{23}^{s} \quad(\text { for } n \geqslant 2) .
\end{aligned}
$$

In the above, $R_{23}^{s}$ is the reflectance of the pump pulse incident at the substrate (layer 2)/ $N_{2}$-purged (layer 3 ) interface from the substrate $\left[\alpha_{\text {inter }}\left(I_{0}\right)=0\right.$ in Eq. (D11b)]. Using Eqs. (D13), (D14a)-(D14c), and (D15), the absorbed pump intensity $\mathcal{I}_{a b}(t)$ is determined by

$$
\begin{aligned}
\mathcal{I}_{a b}(t)= & \mathcal{I}_{0}(t) A_{12}^{s *}\left(F_{0} / 2 \tau_{\text {pump }}\right) \\
& +\sum_{n} \mathcal{I}_{n}(t+n \Delta t) A_{21}^{s *}\left(F_{\mathrm{n}} / 2 \tau_{\text {pump }}\right) .
\end{aligned}
$$

Figures 11(a)-11(f) depict the pump intensity dependence of $\alpha_{\mathrm{inter}}$ and $A_{\mathrm{ij}}^{s}$ for various $\tau_{\mathrm{ie}}$ and $T_{e}$ calculated using Eqs. (D9) and (D14c). Figures 12(a) and 12(b) present the saturation pump intensities $I_{s}$ for $\alpha_{\text {inter }}$ and $A_{12}^{s *}$, respectively, where $I_{s}$ is defined as $\alpha_{\text {inter }}\left(I_{s}\right)=(1 / 2) \alpha_{\text {inter }}(0)$ and $A_{12}^{s *}\left(I_{s}\right)=$ $(1 / 2) A_{12}^{s *}(0)$. Figure 13 shows the absorbed pump fluence in graphene with $\left|\varepsilon_{\mathrm{F}}\right|=0.15$ and $0.43 \mathrm{eV}$, calculated using Eq. (D17).

\section{APPENDIX E: CALCULATION OF THE TRANSIENT THz REFLECTION CHANGE FROM OPTICAL CONDUCTIVITY}

In this Appendix, we explain the calculation procedure of the transient reflection change $\Delta E_{\mathrm{r}}\left(\tau_{1}\right) / E_{0}$ from $\sigma\left(\omega, \tau_{1}\right)$,
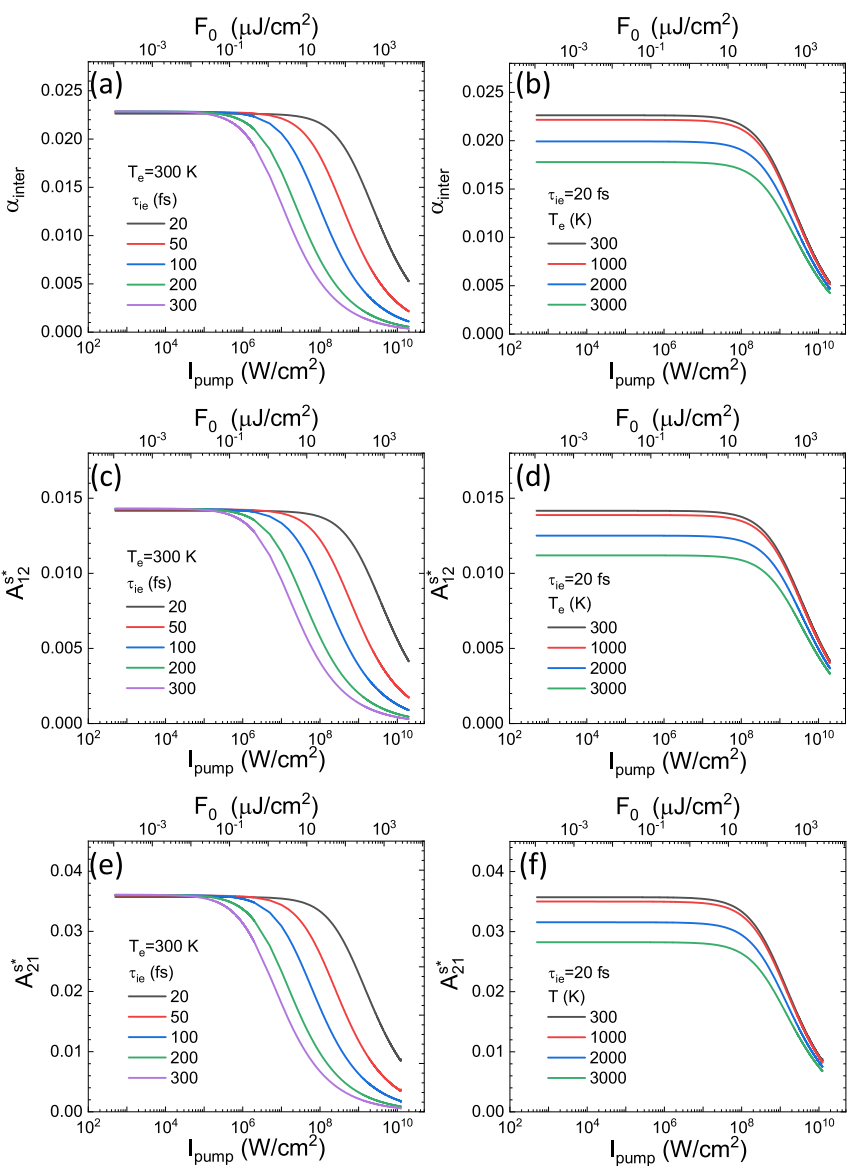

FIG. 11. (a)-(f) Pump intensity $I_{\text {pump }}$ dependence of $\alpha_{\text {inter }}$ and $A_{\mathrm{ij}}^{s}$ at $\theta=60^{\circ}$ in heavily doped graphene with $\left|\varepsilon_{\mathrm{F}}\right|=0.43 \mathrm{eV}$, assuming $\epsilon_{2}=2.4$ for various $\tau_{\text {ie }}$ and $T_{e}$. The $F_{0}=I_{0} \times 2 \tau_{\text {pump }}$ on the upper horizontal axis was calculated by assuming $2 \tau_{\text {pump }}=220 \mathrm{fs}$ in the OPTP experiment.

calculated using the iterative solution of the BTE and the four-temperature model. The reflected $\mathrm{THz}$ electric field in the time domain, $E_{\mathrm{r}}^{\mathrm{s}}\left(\tau_{2}, \tau_{1}\right)$, where $\tau_{2}$ is the probe trigger delay, is determined by the inverse Fourier transformation of the reflected $\mathrm{THz}$ electric field in the frequency domain,
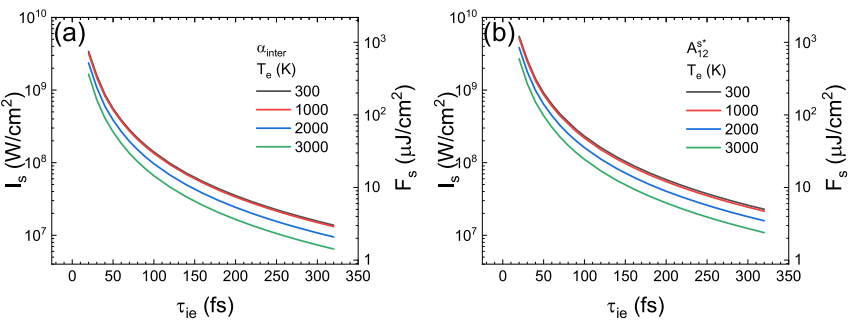

FIG. 12. $T_{e}$ dependence of saturation pump intensity $I_{s}$ of heavily doped graphene with $\left|\varepsilon_{\mathrm{F}}\right|=0.43 \mathrm{eV}$, assuming $\epsilon_{2}=2.4$ for (a) $\alpha_{\text {inter }}$ and (b) $A_{\mathrm{ij}}^{s}$ at $\theta=60^{\circ}$. The $F_{\mathrm{s}}=I_{\mathrm{s}} \times 2 \tau_{\text {pump }}$ on the right vertical axis was calculated by assuming $2 \tau_{\text {pump }}=220 \mathrm{fs}$ in the OPTP experiment. 

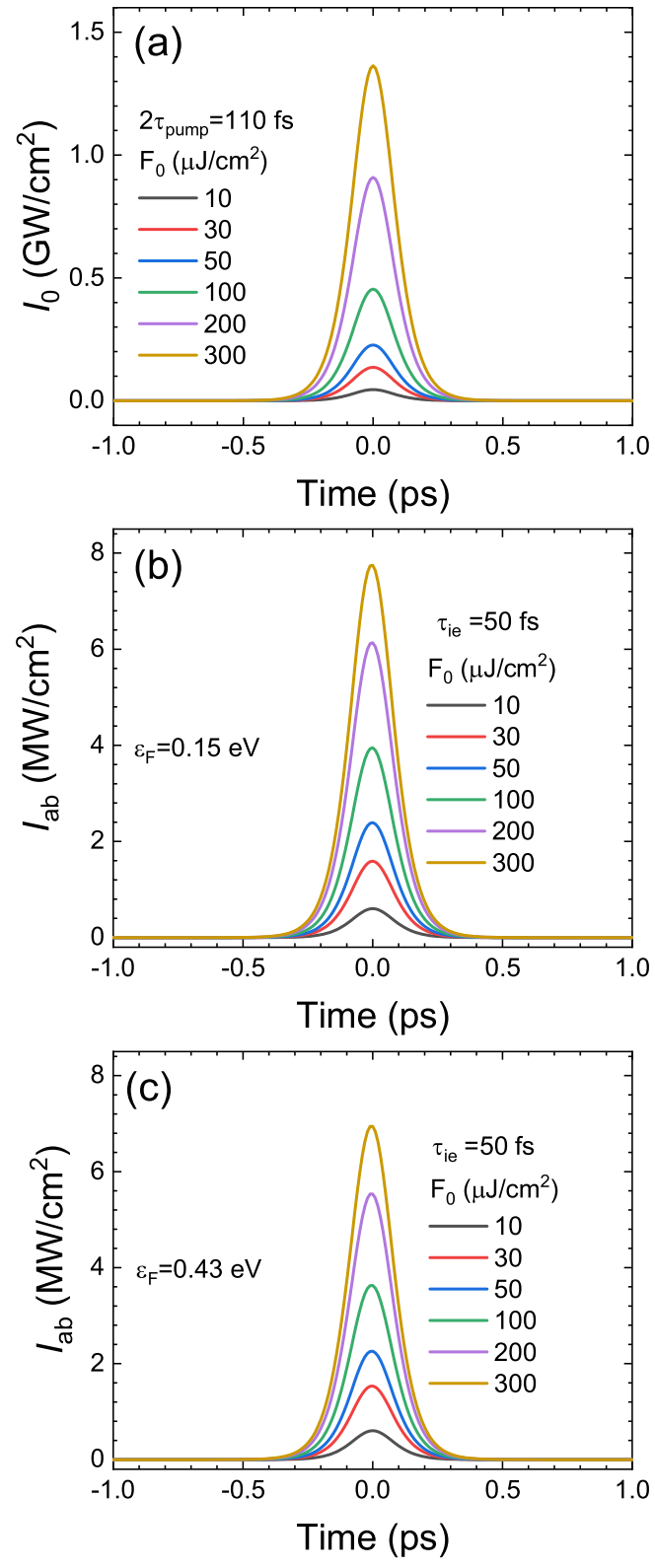

FIG. 13. (a) Envelope function of pump fluence $F_{0}$ incident on graphene at $\theta=60^{\circ}$. Absorbed pump intensity $\mathcal{I}_{\mathrm{ab}}$ in graphene with (b) $\left|\varepsilon_{\mathrm{F}}\right|=0.15$ and (c) $\left|\varepsilon_{\mathrm{F}}\right|=0.43 \mathrm{eV}$ at $T_{e}=295 \mathrm{~K}$ using $\epsilon_{2}=2.4$.

$E_{\mathrm{r}}^{\mathrm{s}}\left(\omega_{\mathrm{THz}}, \tau_{1}\right)$ :

$$
\begin{aligned}
E_{\mathrm{r}}^{\mathrm{s}}\left(\tau_{2}, \tau_{1}\right) & =\int E_{\mathrm{r}}^{\mathrm{s}}\left(\omega_{\mathrm{THz}}, \tau_{1}\right) e^{i \omega_{\mathrm{TH}} \tau_{2}} d \omega_{\mathrm{THz}} \\
& =\int E_{\mathrm{i}}^{\mathrm{s}}\left(\omega_{\mathrm{THz}}\right) r_{\mathrm{s}}\left(\omega_{\mathrm{THz}}, \tau_{1}\right) e^{i \omega_{\mathrm{TH}} \tau_{2}} d \omega_{\mathrm{THz}},
\end{aligned}
$$

where $E_{\mathrm{i}}^{\mathrm{s}}\left(\omega_{\mathrm{THz}}\right)$ is the electric field of the incident $\mathrm{THz}$ pulse in the frequency domain and $r_{s}^{\prime}\left(\omega_{\mathrm{THz}}, \tau_{1}\right)$ is the refection coefficient of the $\mathrm{THz}$ probe by the photoexcited graphene at $\tau_{1}$, which is calculated as a function of $\sigma\left(\omega, \tau_{1}\right)$ by Eq. (B1). The normalized reflection change $\Delta E_{\mathrm{r}}^{\mathrm{s}}\left(\tau_{2}, \tau_{1}\right) / E_{\mathrm{r}}^{\mathrm{s}}\left(\tau_{2}\right)=$ $\Delta E_{\mathrm{r}}\left(\tau_{1}\right) / E_{0}$ as a function of the probe trigger delay $\tau_{2}$ at $\tau_{1}$
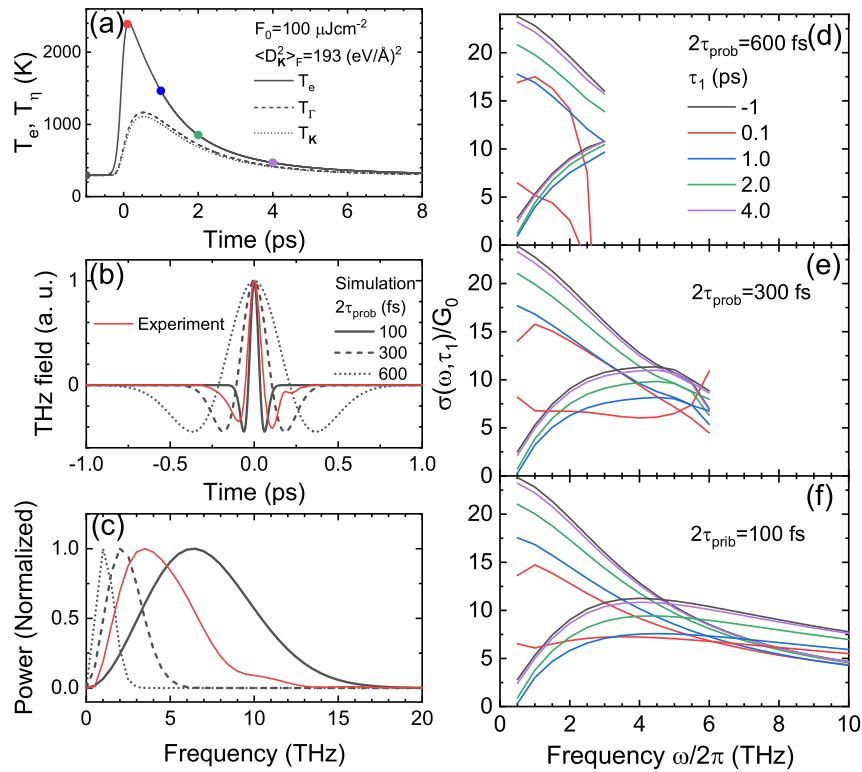

FIG. 14. (a) Temporal evolution of $T_{e}$ and $T_{\eta}$ of heavily doped graphene with $\left|\varepsilon_{\mathrm{F}}\right|=0.43 \mathrm{eV}$. (b) Temporal waveform of THz probe pulse with $2 \tau_{\text {prob }}=100,300$, and 600 (fs) used in the simulation. (c) Corresponding Fourier spectrum of $\mathrm{THz}$ probe pulse. Temporal evolution of $\sigma\left(\omega, \tau_{1}\right)$ at $\tau_{1}=-1.0,0.1,1.0,2.0$, and $4.0 \mathrm{ps}$ calculated using the THz probe with (d) $2 \tau_{1}=600$, (e) $2 \tau_{1}=300$, and (f) $2 \tau_{1}=100 \mathrm{fs}$.

is expressed by

$$
\frac{\Delta E_{\mathrm{r}}\left(\tau_{1}\right)}{E_{0}} \equiv \frac{\Delta E_{\mathrm{r}}^{\mathrm{s}}\left(\tau_{2}, \tau_{1}\right)}{E_{\mathrm{r}}^{\mathrm{s}}\left(\tau_{2}\right)}=\frac{E_{\mathrm{r}}^{\mathrm{s}}\left(\tau_{2}, \tau_{1}\right)-E_{\mathrm{r}}^{\mathrm{s}}\left(\tau_{2}\right)}{E_{\mathrm{r}}^{\mathrm{s}}\left(\tau_{2}\right)} .
$$

In the above, $E_{\mathrm{r}}^{s}\left(\tau_{2}\right)$ is the reflected $\mathrm{THz}$ field through the graphene sample without pump fluence. We define the transient reflectivity $\Delta E_{\mathrm{r}}\left(\tau_{1}\right) / E_{0} \equiv \Delta E_{\mathrm{r}}^{\mathrm{s}}\left(\tau_{2}, \tau_{1}\right) / E_{\mathrm{r}}^{\mathrm{s}}\left(\tau_{2}\right)$ at $\tau_{2}=0$ ps when the peak amplitude of $E_{\mathrm{r}}^{s}\left(\tau_{2}\right)$ takes the maximum amplitude.

Figures 14(a), 14(b), and 14(c) depict the temporal evolution of $T_{e}$ and $T_{\eta}$ of the photoexcited graphene, the temporal waveforms of the THz probe pulse, and the Fourier spectra of the $\mathrm{THz}$ probe pulse, respectively, used in the calculation of $\sigma\left(\omega, \tau_{1}\right)[43]$ in Figs. 14(d)-14(f) for $\left\langle D_{\mathbf{K}}^{2}\right\rangle_{\mathrm{F}}=193(\mathrm{eV} / \AA)^{2}$. The $\sigma\left(\omega, \tau_{1}\right)$ values are plotted only in the frequency range corresponding to the bandwidth of the $\mathrm{THz}$ probe, because the numerical error occurs outside the frequency of the bandwidth. $\sigma\left(\omega, \tau_{1}\right)$ is strongly dependent on the waveform of the $\mathrm{THz}$ probe pulse, and non-Drude frequency dependence clearly appears at $\tau_{1}=0.1 \mathrm{ps}$ when the carrier distribution and scattering rate change very rapidly during the $\mathrm{THz}$ probing time owing to the photoexcitation. Figure 15 depicts the $\left\langle D_{\mathbf{K}}^{2}\right\rangle_{\mathrm{F}}$ dependence of $\Delta E_{\mathrm{r}}\left(\tau_{1}\right) / E_{0}$ for different $2 \tau_{\text {prob }}$ values calculated using Eq. (E2). The $\Delta E_{\mathrm{r}}\left(\tau_{1}\right) / E_{0}$ reflects the change in the $\sigma\left(\omega, \tau_{1}\right)$ around the center frequency of the $\mathrm{THz}$ probe pulse, and the peak value becomes higher depending on the $\left\langle D_{\mathbf{K}}^{2}\right\rangle_{\mathrm{F}}$. 


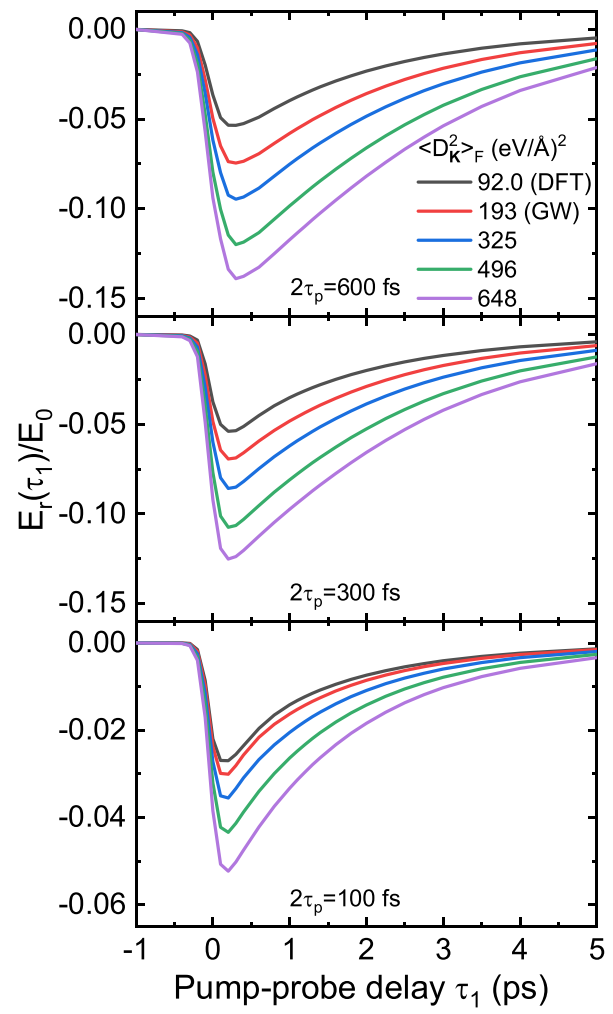

FIG. 15. $\left\langle D_{\mathbf{K}}^{2}\right\rangle_{\mathrm{F}}$ dependence of $\Delta E_{\mathrm{r}}\left(\tau_{1}\right) / E_{0}$ of heavily doped graphene calculated using the $\mathrm{THz}$ probe pulse with $2 \tau_{\text {prob }}=600$, 300 , and 100 fs for $F_{0}=100 \mu \mathrm{J} / \mathrm{cm}^{2}$.

\section{APPENDIX F: DETERMINATION OF THE EPC CONSTANT FROM THE SIMULATION}

In this Appendix, we present the procedure to determine the EPC constant $\left\langle D_{\mathbf{K}}^{2}\right\rangle_{\mathrm{F}}$ near the $\mathbf{K}$ point via the numerical simulation based on the Boltzmann transport equation. In the numerical simulation, we used four unknown free parameters associated with carrier scattering $\left(\left\langle D_{\mathbf{K}}^{2}\right\rangle_{\mathrm{F}}, J_{\mathrm{a}}, \rho_{\mathrm{s}}, n_{\mathrm{i}}\right)$ and two unknown free parameters associated with the optical phonon decay time $\left(\tau_{\mathrm{ph}}\right)$ and saturable absorption $\left(\tau_{\mathrm{ie}}\right)$ for the fitting of $\Delta E_{\mathrm{r}}\left(\tau_{1}\right) / E_{0}$ and $\sigma_{\mathrm{dc}}$. Here, the reported values of $J_{\mathrm{a}}$ and $\rho_{\mathrm{s}}$ are equal to $10-30 \mathrm{eV}[71,72,74-76,82,90-99]$ and 40 $100 \Omega[70,71,85,86]$, respectively. Both $J_{\mathrm{a}}$ and $\rho_{\mathrm{s}}$ contribute to the hot-carrier-cooling process through the defect-induced supercollision (SC) process. The energy loss rate $J_{\mathrm{sc}}$ of the SC process [Eqs. (9a) and (9b)] [59,102] is

$$
J_{\mathrm{sc}} \approx 8.8 \times 10^{14} \frac{J_{\mathrm{a}}^{2}}{\mu_{\text {Defect }}}\left(T_{e}^{3}-T_{\mathrm{ac}}^{3}\right) \mathrm{eV} / \mathrm{s} .
$$

Here, $\mu_{\text {Defect }}$ is the defect-limited carrier mobility which is inversely proportional to $\rho_{\mathrm{s}}$. However, $n_{\mathrm{i}}$ is not responsible for the temporal evolution of $T_{e}$ and $T_{\eta}$ because the charged impurity scattering is elastic. Furthermore, the charged impurity scattering less affects the temperature dependence of the scattering rate through the change in the carrier distribution and screening effect, as seen in Fig. 1(d). Therefore we conducted the numerical calculation under the minimum $\left(J_{\mathrm{a}}=10 \mathrm{eV}\right.$, $\left.\rho_{\mathrm{s}}=40 \Omega\right)$ and maximum $\left(J_{\mathrm{a}}=30 \mathrm{eV}, \rho_{\mathrm{s}}=100 \Omega\right)$ SC process conditions to understand the parameter dependence.

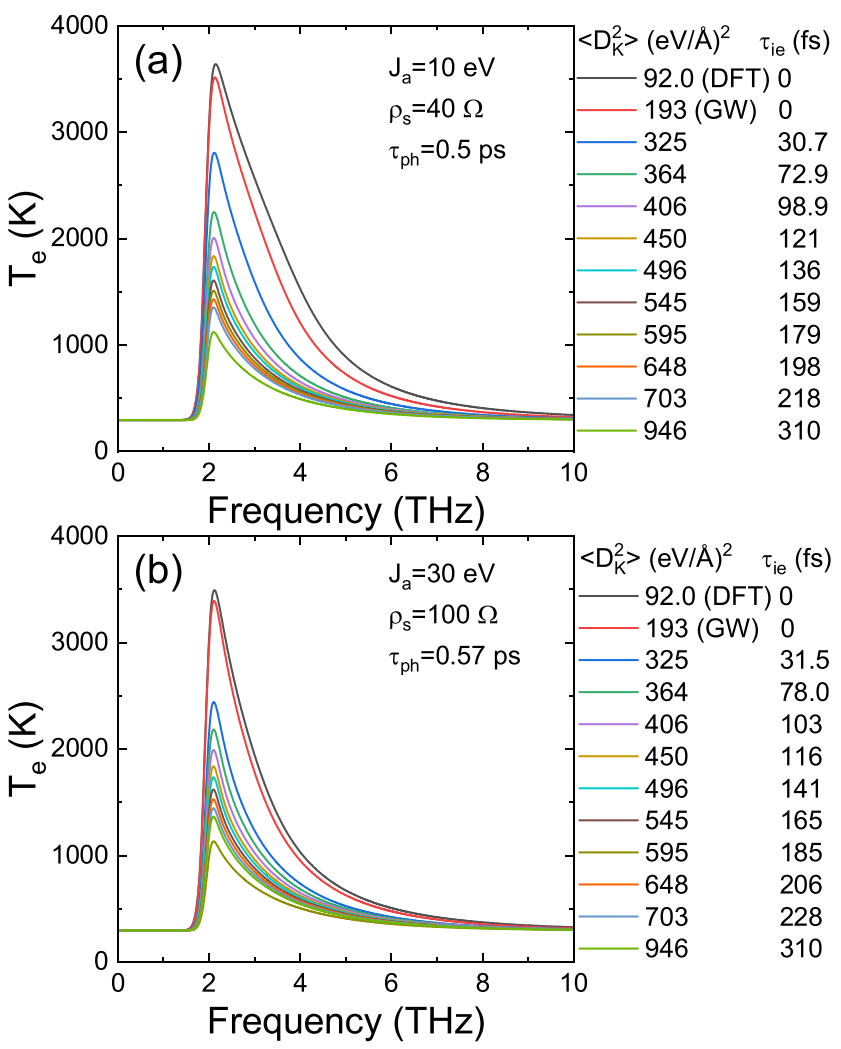

FIG. 16. $\left\langle D_{\mathbf{K}}^{2}\right\rangle_{\mathrm{F}}$ dependence of $T_{e}$ of heavily doped graphene for $F_{0}=200 \mu \mathrm{J} / \mathrm{cm}^{2}$ under different parameter conditions. (a) $J_{\mathrm{a}}=$ $10 \mathrm{eV}, \rho_{\mathrm{s}}=40 \Omega$, and $\tau_{\mathrm{ph}}=0.5$ ps. (b) $J_{\mathrm{a}}=30 \mathrm{eV}, \rho_{\mathrm{s}}=100 \Omega$, and $\tau_{\mathrm{ph}}=0.57 \mathrm{ps}$.

First, we determined the $n_{\mathrm{i}}$ value from the dc conductivity $\sigma_{\mathrm{dc}}$. Since $\sigma_{\mathrm{dc}}$ is a function of $\left\langle D_{\mathbf{K}}^{2}\right\rangle_{\mathrm{F}}, J_{\mathrm{a}}, \rho_{\mathrm{s}}$, and $n_{\mathrm{i}}, n_{\mathrm{i}}$ can be determined from the fitting of $\sigma_{\mathrm{dc}}$. Hence we fitted $\Delta E_{\mathrm{r}}\left(\tau_{1}\right) / E_{0}$ for $F_{0}=200 \mu \mathrm{J} / \mathrm{cm}^{2}$ using $\left\langle D_{\mathbf{K}}^{2}\right\rangle_{\mathrm{F}}, \tau_{\text {ie }}$, and $\tau_{\mathrm{ph}}$ as free parameters. Figures 16(a) and 16(b) show the temporal evolution of $T_{e}$ used in the fitting of $\Delta E_{\mathrm{r}}\left(\tau_{1}\right) / E_{0}$ under the minimum and maximum SC conditions for $F_{0}=200 \mu \mathrm{J} / \mathrm{cm}^{2}$. The multiple reflections of pump pulses inside the PET substrate were not included. Since the negative photoconductivity of hot carriers in heavily doped graphene originates from the temperature dependence of the Drude weight and carrierscattering rate, a higher $\left\langle D_{\mathbf{K}}^{2}\right\rangle_{\mathrm{F}}$ results in higher $\tau_{\mathrm{ie}}$ values leading to stronger saturable absorption and a lower peak value of $T_{e}$. Figure 16 shows that for a low $\left\langle D_{\mathbf{K}}^{2}\right\rangle_{\mathrm{F}}$, the peak of the $T_{e}$ curve is broad because the energy loss rate $R_{\mathrm{K}}^{\mathrm{Net}} \hbar \omega_{\mathrm{K}}$ of hot carriers for optical phonons is small.

Figures 17(a) and 17(b) show the fitting of $\Delta E_{\mathrm{r}}\left(\tau_{1}\right) / E_{0}$ at the minimum and maximum SC conditions, respectively, for $F_{0}=50,100$, and $200 \mu \mathrm{J} / \mathrm{cm}^{2}$. The fitting results indicate that the optical phonon decay time $\tau_{\mathrm{ph}}$ increases with $F_{0}$ and $J_{\mathrm{sc}}$. For $\left\langle D_{\mathrm{K}}^{2}\right\rangle_{\mathrm{F}} \leqslant 405(\mathrm{eV} / \AA)^{2}$, the first and second sharp peaks of the calculated curves are significantly broader than those of the experimentally determined curves due to the longer relaxation time of $T_{e}$. For $\left\langle D_{\mathbf{K}}^{2}\right\rangle_{\mathrm{F}} \geqslant 450(\mathrm{eV} / \AA)^{2}$, the calculations fit closely with the experimental results, which indicates that $\left\langle D_{\mathbf{K}}^{2}\right\rangle_{\mathrm{F}}=450(\mathrm{eV} / \AA)^{2}$ is the lower limit value under both conditions. 

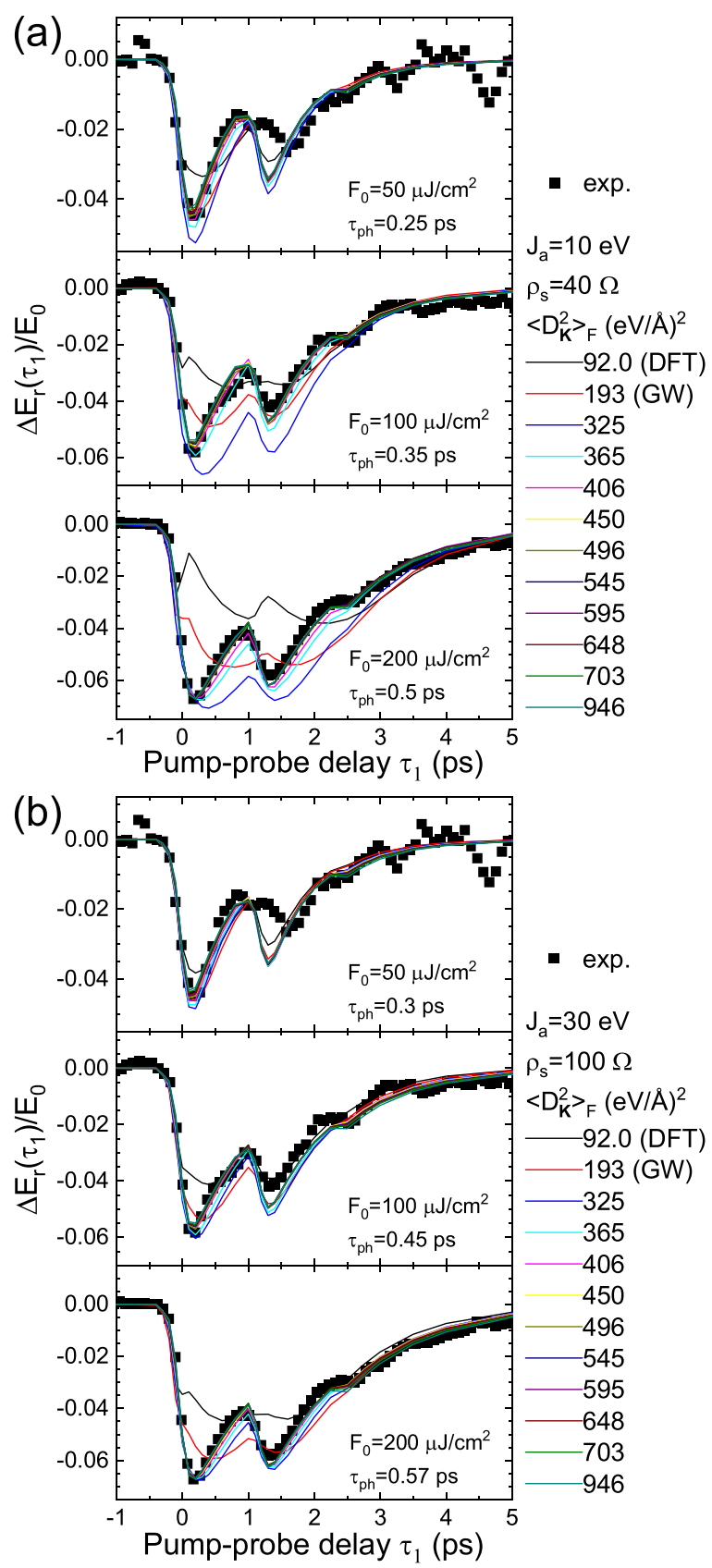

FIG. 17. Fitting of $\Delta E_{\mathrm{r}}\left(\tau_{1}\right) / E_{0}$ of heavily doped graphene under different parameter conditions. (a) $J_{\mathrm{a}}=10 \mathrm{eV}, \rho_{\mathrm{s}}=40 \Omega$, and $\tau_{\mathrm{ph}}=$ 0.5 ps. (b) $J_{\mathrm{a}}=30 \mathrm{eV}, \rho_{\mathrm{s}}=100 \Omega$, and $\tau_{\mathrm{ph}}=0.57$ ps.

To estimate the higher limit value of $\left\langle D_{\mathbf{K}}^{2}\right\rangle_{\mathrm{F}}$, we compared the experimentally determined $\sigma\left(\omega, \tau_{1}\right)=\sigma_{1}\left(\omega, \tau_{1}\right)+$ $i \sigma_{2}\left(\omega, \tau_{1}\right)$ values with the corresponding calculated values under the minimum and maximum SC conditions for $F_{0}=300$ $\mu \mathrm{J} / \mathrm{cm}^{2}$; the results are shown in Figs. 18(a) and 18(b), respectively. Both the calculated $\sigma_{1}\left(\omega, \tau_{1}\right)$ and $\sigma_{2}\left(\omega, \tau_{1}\right)$ values slightly decrease as $\left\langle D_{\mathbf{K}}^{2}\right\rangle_{\mathrm{F}}$ increases, which indicates that both the amplitude reflectance $\left|r_{\mathrm{s}}^{\prime}\right|$ and phase shift $\arg \left(r_{\mathrm{s}}^{\prime}\right)$ in Eq. (B1) decrease. Since $\Delta E_{\mathrm{r}}\left(\tau_{1}\right) / E_{0}$ was measured at a fixed $\mathrm{THz}$ probe-trigger pulse delay $\tau_{2}=0 \mathrm{ps}$, when the THz pulse amplitude is maximum, a small phase $\operatorname{shift} \arg \left(r_{\mathrm{s}}^{\prime}\right)$ leads to a small reduction in $\Delta E_{\mathrm{r}}\left(\tau_{1}\right) / E_{0}$. Consequently, the
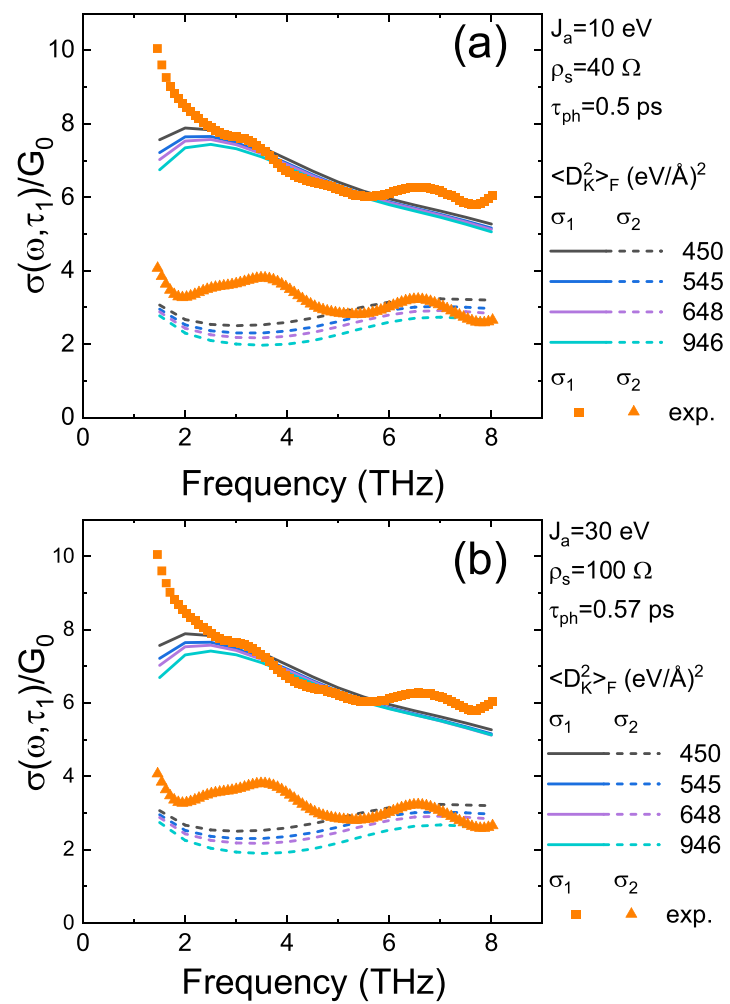

FIG. 18. Comparison of $\sigma_{1}\left(\omega, \tau_{1}\right)$ of heavily doped graphene for $F_{0}=200 \mu \mathrm{J} / \mathrm{cm}^{2}$ under different parameter conditions. (a) $J_{\mathrm{a}}=$ $10 \mathrm{eV}, \rho_{\mathrm{s}}=40 \Omega$, and $\tau_{\mathrm{ph}}=0.5$ ps. (b) $J_{\mathrm{a}}=30 \mathrm{eV}, \rho_{\mathrm{s}}=100 \Omega$, and $\tau_{\mathrm{ph}}=0.57 \mathrm{ps}$.

TABLE III. Parameters used in the calculation of $\Delta E_{\mathrm{r}}\left(\tau_{1}\right) / E_{0}$ and $\sigma\left(\omega, \tau_{1}\right) .\left\langle D_{\Gamma}^{2}\right\rangle_{\mathrm{F}}=45.6(\mathrm{eV} / \AA)^{2}$.

\begin{tabular}{|c|c|c|c|c|}
\hline$\left\langle D_{\mathrm{K}}^{2}\right\rangle_{\mathrm{F}}(\mathrm{eV} / \AA)^{2}$ & $\tau_{\text {ie }}(\mathrm{fs})$ & $n_{\mathrm{i}}\left(\mathrm{cm}^{-2}\right)$ & $J_{\mathrm{a}}(\mathrm{eV})$ & $\rho_{\mathrm{s}}(\Omega)$ \\
\hline 92.0 & & $1.15 \times 10^{12}$ & 10 & 40 \\
\hline 193 & & $1.13 \times 10^{12}$ & 10 & 40 \\
\hline 325 & 52.5 & $1.11 \times 10^{12}$ & 10 & 40 \\
\hline 365 & 75.0 & $1.10 \times 10^{12}$ & 10 & 40 \\
\hline 406 & 96.0 & $1.09 \times 10^{12}$ & 10 & 40 \\
\hline 450 & 116 & $1.09 \times 10^{12}$ & 10 & 40 \\
\hline 496 & 131 & $1.08 \times 10^{12}$ & 10 & 40 \\
\hline 545 & 151 & $1.07 \times 10^{12}$ & 10 & 40 \\
\hline 595 & 170 & $1.06 \times 10^{12}$ & 10 & 40 \\
\hline 648 & 189 & $1.06 \times 10^{12}$ & 10 & 40 \\
\hline 703 & 210 & $1.05 \times 10^{12}$ & 10 & 40 \\
\hline 946 & 299 & $1.01 \times 10^{12}$ & 10 & 40 \\
\hline 92.0 & & $4.40 \times 10^{12}$ & 30 & 100 \\
\hline 193 & & $4.39 \times 10^{12}$ & 30 & 100 \\
\hline 325 & 30.7 & $4.36 \times 10^{12}$ & 30 & 100 \\
\hline 365 & 72.9 & $4.35 \times 10^{12}$ & 30 & 100 \\
\hline 406 & 98.9 & $4.35 \times 10^{12}$ & 30 & 100 \\
\hline 450 & 121 & $4.34 \times 10^{12}$ & 30 & 100 \\
\hline 496 & 136 & $4.33 \times 10^{12}$ & 30 & 100 \\
\hline 545 & 159 & $4.33 \times 10^{12}$ & 30 & 100 \\
\hline 595 & 179 & $4.32 \times 10^{12}$ & 30 & 100 \\
\hline 648 & 198 & $4.31 \times 10^{12}$ & 30 & 100 \\
\hline 703 & 219 & $4.30 \times 10^{12}$ & 30 & 100 \\
\hline 946 & 310 & $4.27 \times 10^{12}$ & 30 & 100 \\
\hline
\end{tabular}


calculated $\sigma\left(\omega, \tau_{1}\right)$ yields similar peak values of $\Delta E_{\mathrm{r}}\left(\tau_{1}\right) / E_{0}$ for $\left\langle D_{\mathbf{K}}^{2}\right\rangle_{\mathrm{F}}=450-946(\mathrm{eV} / \AA)^{2}$ as seen in Fig. 17. However, because the deviation in $\sigma_{2}\left(\omega, \tau_{1}\right)$ between the experiment and calculation increases with $\left\langle D_{\mathbf{K}}^{2}\right\rangle_{\mathrm{F}}$, we estimated the EPC constant to be $\left\langle D_{\mathbf{K}}^{2}\right\rangle_{\mathrm{F}}=450 \pm 45(\mathrm{eV} / \AA)^{2}$ under both SC conditions. The parameters used in the simulations for the minimum and maximum SC conditions are summarized in Table III.
[1] N. Del Fatti, C. Voisin, M. Achermann, S. Tzortzakis, D. Christofilos, and F. Vallée, Nonequilibrium electron dynamics in noble metals, Phys. Rev. B 61, 16956 (2000).

[2] A. J. Nozik, Spectroscopy and hot electron relaxation dynamics in semiconductor quantum wells and quantum dots, Annu. Rev. Phys. Chem. 52, 193 (2001).

[3] F. Xia, T. Mueller, Y.-M. Lin, A. Valdes-Garcia, and P. Avouris, Ultrafast graphene photodetector, Nat. Nanotechnol. 4, 839 (2009).

[4] W. K. Tse and S. Das Sarma, Energy relaxation of hot Dirac fermions in graphene, Phys. Rev. B 79, 235406 (2009).

[5] S. Berciaud, M. Y. Han, K. F. Mak, L. E. Brus, P. Kim, and T. F. Heinz, Electron and Optical Phonon Temperatures in Electrically Biased Graphene, Phys. Rev. Lett. 104, 227401 (2010).

[6] X. Xu, N. M. Gabor, J. S. Alden, A. M. Van Der Zande, and P. L. McEuen, Photo-thermoelectric effect at a graphene interface junction, Nano Lett. 10, 562 (2010).

[7] N. M. Gabor, J. C. W. Song, N. L. N. Q. Ma, T. Taychatanapat, K. Watanabe, T. Taniguchi, L. S. Levitov, and P. J. Herrero, Hot carrier-assisted intrinsic photoresponse in graphene, Science 334, 648 (2011).

[8] D. Sun, G. Aivazian, A. M. Jones, J. S. Ross, W. Yao, D. Cobden, and $\mathrm{X}$. Xu, Ultrafast hot-carrier-dominated photocurrent in graphene, Nat. Nanotechnol. 7, 114 (2012).

[9] S. Wu, W.-T. Liu, X. Liang, P. J. Schuck, F. Wang, Y. R. Shen, and M. Salmeron, Hot phonon dynamics in graphene, Nano Lett. 12, 5495 (2012).

[10] C. H. Liu, Y. C. Chang, T. B. Norris, and Z. Zhong, Graphene photodetectors with ultra-broadband and high responsivity at room temperature, Nat. Nanotechnol. 9, 273 (2014).

[11] J. C. Song and L. S. Levitov, Energy flows in graphene: Hot carrier dynamics and cooling, J. Phys.: Condens. Matter 27, 164201 (2015).

[12] K. J. Tielrooij, M. Massicotte, L. Piatkowski, A. Woessner, Q. Ma, P. Jarillo-Herrero, N. F. Hulst, and F. H. Koppens, Hot-carrier photocurrent effects at graphene-metal interfaces, J. Phys.: Condens. Matter 27, 164207 (2015).

[13] A. Stange, C. Sohrt, L. X. Yang, G. Rohde, K. Janssen, P. Hein, L. P. Oloff, K. Hanff, K. Rossnagel, and M. Bauer, Hot electron cooling in graphite: Supercollision versus hot phonon decay, Phys. Rev. B 92, 184303 (2015).

[14] G. Kané, M. Lazzeri, and F. Mauri, High-field transport in graphene: The impact of Zener tunneling, J. Phys.: Condens. Matter 27, 164205 (2015).

[15] C. B. McKitterick, D. E. Prober, H. Vora, and X. Du, Ultrasensitive graphene far-infrared power detectors, J. Phys.: Condens. Matter 27, 164203 (2015)

[16] F. Bonaccorso, L. Colombo, G. Yu, M. Stoller, V. Tozzini, A. C. Ferrari, R. S. Ruoff, and V. Pellegrini, Graphene, related two-dimensional crystals, and hybrid systems for energy conversion and storage, Science 347, 41 (2015).
[17] Q. Y. Li, T. Feng, W. Okita, Y. Komori, H. Suzuki, T. Kato, T. Kaneko, T. Ikuta, X. Ruan, and K. Takahashi, Enhanced thermoelectric performance of as-grown suspended graphene nanoribbons, ACS Nano 13, 9182 (2019).

[18] Y. Chen, Y. Li, Y. Zhao, H. Zhou, and H. Zhu, Highly efficient hot electron harvesting from graphene before electron-hole thermalization, Sci. Adv. 5, eaax9958 (2019).

[19] M. L. Lin, Y. Zhou, J. B. Wu, X. Cong, X. L. Liu, J. Zhang, H. Li, W. Yao, and P. H. Tan, Cross-dimensional electron-phonon coupling in van der Waals heterostructures, Nat. Commun. 10, 2419 (2019).

[20] Y. Lin, Q. Ma, P. C. Shen, B. Ilyas, Y. Bie, A. Liao, E. Ergeçen, B. Han, N. Mao, X. Zhang, X. Ji, Y. Zhang, J. Yin, S. Huang, M. Dresselhaus, N. Gedik, P. Jarillo-Herrero, X. Ling, J. Kong, and T. Palacios, Asymmetric hot-carrier thermalization and broadband photoresponse in graphene-2D semiconductor lateral heterojunctions, Sci. Adv. 5, eaav1493 (2019).

[21] Y. R. Kim, T. L. Phan, Y. S. Shin, W. T. Kang, U. Y. Won, I. Lee, J. E. Kim, K. Kim, Y. H. Lee, and W. J. Yu, Unveiling the hot carrier distribution in vertical graphene/h-BN/Au van der Waals heterostructures for high-performance photodetector, ACS Appl. Mater. Interfaces 12, 10772 (2020).

[22] Y. Kim, J. H. Kim, Y. H. Lee, M. D. Tran, S. G. Lee, S. Jeon, S. T. Kim, H. Kim, V. L. Nguyen, S. Adhikari, S. Woo, and H. C. Park, Decelerated hot carrier cooling in graphene via nondissipative carrier injection from $\mathrm{MoS}_{2}$, ACS Nano 14, 13905 (2020).

[23] M. Massicotte, G. Soavi, A. Principi, and K. J. Tielrooij, Hot carriers in graphene-fundamentals and applications, Nanoscale 13, 8376 (2021).

[24] E. A. A. Pogna, X. Jia, A. Principi, A. Block, L. Banszerus, J. Zhang, X. Liu, T. Sohier, S. Forti, K. Soundarapandian, B. Terrés, J. D. Mehew, C. Trovatello, C. Coletti, F. H. L. Koppens, M. Bonn, H. I. Wang, N. van Hulst, M. J. Verstraete, H. Peng et al., Hot-carrier cooling in high-quality graphene is intrinsically limited by optical phonons, ACS Nano 15, 11285 (2021).

[25] D. Sun, Z.-K. Wu, C. Divin, X. Li, C. Berger, W. A. de Heer, P. N. First, and T. B. Norris, Ultrafast Relaxation of Excited Dirac Fermions in Epitaxial Graphene Using Optical Differential Transmission Spectroscopy, Phys. Rev. Lett. 101, 157402 (2008).

[26] I. Gierz, J. C. Petersen, M. Mitrano, C. Cacho, I. C. Turcu, E. Springate, A. Stöhr, A. Köhler, U. Starke, and A. Cavalleri, Snapshots of non-equilibrium Dirac carrier distributions in graphene, Nat. Mater. 12, 1119 (2013).

[27] J. C. Johannsen, S. Ulstrup, F. Cilento, A. Crepaldi, M. Zacchigna, C. Cacho, I. C. E. Turcu, E. Springate, F. Fromm, C. Raidel, T. Seyller, F. Parmigiani, M. Grioni, and P. Hofmann, Direct View of Hot Carrier Dynamics in Graphene, Phys. Rev. Lett. 111, 027403 (2013). 
[28] P. A. George, J. Strait, J. Dawlaty, S. Shivaraman, M. Chandrashekhar, F. Rana, and M. G. Spencer, Ultrafast optical-pump terahertz-probe spectroscopy of the carrier relaxation and recombinataion dynamics in epitaxial graphene, Nano Lett. 8, 4248 (2008).

[29] J. H. Strait, H. Wang, S. Shivaraman, V. Shields, M. Spencer, and F. Rana, Very slow cooling dynamics of photoexcited carriers in graphene observed by optical-pump terahertz-probe spectroscopy, Nano Lett. 11, 4902 (2011).

[30] S. Boubanga-Tombet, S. Chan, T. Watanabe, A. Satou, V. Ryzhii, and T. Otsuji, Ultrafast carrier dynamics and terahertz emission in optically pumped graphene at room temperature, Phys. Rev. B 85, 035443 (2012).

[31] C. J. Docherty, C.-T. Lin, H. J. Joyce, R. J. Nicholas, L. M. Herz, L.-J. Li, and M. B. Johnston, Extreme sensitivity of graphene photoconductivity to environmental gases, Nat. Commun. 3, 1228 (2012).

[32] A. J. Frenzel, C. H. Lui, W. Fang, N. L. Nair, P. K. Herring, P. Jarillo-Herrero, J. Kong, and N. Gedik, Observation of suppressed terahertz absorption in photoexcited graphene, Appl. Phys. Lett. 102, 113111 (2013).

[33] G. Jnawali, Y. Rao, H. Yan, and T. F. Heinz, Observation of a transient decrease in terahertz conductivity of single-layer graphene induced by ultrafast optical excitation, Nano Lett. 13, 524 (2013).

[34] K.-C. Lin, M.-Y. Li, L. J. Li, D. C. Ling, C. C. Chi, and J.C. Chen, Ultrafast dynamics of hot electrons and phonons in chemical vapor deposited graphene, J. Appl. Phys. (Melville, NY) 113, 133511 (2013).

[35] K. J. Tielrooij, J. C. Song, S. A. Jensen, A. Centeno, A. Pesquera, A. Zurutuza Elorza, M. Bonn, L. S. Levitov, and F. H. Koppens, Photoexcitation cascade and multiple hotcarrier generation in graphene, Nat. Phys. 9, 248 (2013).

[36] A. J. Frenzel, C. H. Lui, Y. C. Shin, J. Kong, and N. Gedik, Semiconducting-to-Metallic Photoconductivity Crossover and Temperature-Dependent Drude Weight in Graphene, Phys. Rev. Lett. 113, 056602 (2014).

[37] S. F. Shi, T.-T. Tang, B. Zeng, L. Ju, Q. Zhou, A. Zettl, and F. Wang, Controlling graphene ultrafast hot carrier response from metal-like to semiconductor-like by electrostatic gating, Nano Lett. 14, 1578 (2014).

[38] S. A. Jensen, Z. Mics, I. Ivanov, H. S. Varol, D. Turchinovich, F. H. L. Koppens, M. Bonn, and K. J. Tielrooij, Competing ultrafast energy relaxation pathways in photoexcited graphene, Nano Lett. 14, 5839 (2014)

[39] S. Kar, D. R. Mohapatra, E. Freysz, and A. K. Sood, Tuning photoinduced terahertz conductivity in monolayer graphene: Optical-pump terahertz-probe spectroscopy, Phys. Rev. B 90, 165420 (2014).

[40] J. N. Heyman, J. D. Stein, Z. S. Kaminski, A. R. Banman, A. M. Massari, and J. T. Robinson, Carrier heating and negative photoconductivity in graphene, J. Appl. Phys. (Melville, NY) 117, 015101 (2015).

[41] M. T. Mihnev, F. Kadi, C. J. Divin, T. Winzer, S. Lee, C.-H. Liu, Z. Zhong, C. Berger, W. A. de Heer, E. Malic, A. Knorr, and T. B. Norris, Microscopic origins of the terahertz carrier relaxation and cooling dynamics in graphene, Nat. Commun. 7, 11617 (2016).

[42] A. Tomadin, S. M. Hornett, H. I. Wang, E. M. Alexeev, A. Candini, C. Coletti, D. Turchinovich, M. Kläui, M. Bonn,
F. H. L. Koppens, E. Hendry, M. Polini, and K.-J. Tielrooij, The ultrafast dynamics and conductivity of photoexcited graphene at different Fermi energies, Sci. Adv. 4, eaar5313 (2018).

[43] M. Yamashita and C. Otani, Intrinsic and extrinsic effects on intraband optical conductivity of hot carriers in photoexcited graphene, Phys. Rev. Research 3, 013150 (2021).

[44] S. Piscanec, M. Lazzeri, F. Mauri, A. C. Ferrari, and J. Robertson, Kohn Anomalies and Electron-Phonon Interactions in Graphite, Phys. Rev. Lett. 93, 185503 (2004).

[45] S. Baroni, S. D. Gironcoli, A. D. Corso, S. Scuola, I. Superiore, I. Istituto, F. Materia, I. Trieste, and P. Giannozzi, Phonons and related crystal properties from density-functional perturbation theory, Rev. Mod. Phys. 73, 515 (2001).

[46] S. Pisana, M. Lazzeri, C. Casiraghi, K. S. Novoselov, A. K. Geim, A. C. Ferrari, and F. Mauri, Breakdown of the Adiabatic Born-Oppenheimer Approximation in Graphene, Nat. Mater. 6, 198 (2007).

[47] M. Lazzeri, C. Attaccalite, L. Wirtz, and F. Mauri, Impact of the electron-electron correlation on phonon dispersion: Failure of LDA and GGA DFT functionals in graphene and graphite, Phys. Rev. B 78, 081406(R) (2008).

[48] M. Calandra and F. Mauri, Electron-phonon coupling and electron self-energy in electron-doped graphene: Calculation of angular-resolved photoemission spectra, Phys. Rev. B 76, 205411 (2007).

[49] D. M. Basko, S. Piscanec, and A. C. Ferrari, Electron-electron interactions and doping dependence of the two-phonon Raman intensity in graphene, Phys. Rev. B 80, 165413 (2009).

[50] A. C. Ferrari, J. C. Meyer, V. Scardaci, C. Casiraghi, M. Lazzeri, F. Mauri, S. Piscanec, D. Jiang, K. S. Novoselov, S. Roth, and A. K. Geim, Raman Spectrum of Graphene and Graphene Layers, Phys. Rev. Lett. 97, 187401 (2006).

[51] S. Berciaud, S. Ryu, L. E. Brus, and T. F. Heinz, Probing the intrinsic properties of exfoliated graphene: Raman spectroscopy of free-standing monolayers, Nano Lett. 9, 346 (2009).

[52] A. Grüneis, J. Serrano, A. Bosak, M. Lazzeri, S. L. Molodtsov, L. Wirtz, C. Attaccalite, M. Krisch, A. Rubio, F. Mauri, and T. Pichler, Phonon surface mapping of graphite: Disentangling quasi-degenerate phonon dispersions, Phys. Rev. B 80, 085423 (2009).

[53] D. M. Basko and I. L. Aleiner, Interplay of Coulomb and electron-phonon interactions in graphene, Phys. Rev. B 77, 041409(R) (2008).

[54] G. Onida, L. Reining, and A. Rubio, Electronic excitations: density-functional versus many-body Green's-function approaches, Rev. Mod. Phys. 74, 601 (2002).

[55] A. Grüneis, C. Attaccalite, T. Pichler, V. Zabolotnyy, H. Shiozawa, S. L. Molodtsov, D. Inosov, A. Koitzsch, M. Knupfer, J. Schiessling, R. Follath, R. Weber, P. Rudolf, L. Wirtz, and A. Rubio, Electron-Electron Correlation in Graphite: A Combined Angle-Resolved Photoemission and First-Principles Study, Phys. Rev. Lett. 100, 037601 (2008).

[56] T. Kampfrath, L. Perfetti, F. Schapper, C. Frischkorn, and M. Wolf, Strongly Coupled Optical Phonons in the Ultrafast Dynamics of the Electronic Energy and Current Relaxation in Graphite, Phys. Rev. Lett. 95, 187403 (2005). 
[57] D. Brida, A. Tomadin, C. Manzoni, Y. J. Kim, A. Lombardo, S. Milana, R. R. Nair, K. S. Novoselov, A. C. Ferrari, G. Cerullo, and M. Polini, Ultrafast collinear scattering and carrier multiplication in graphene, Nat. Commun. 4, 1987 (2013).

[58] R. Bistritzer and A. H. MacDonald, Electronic Cooling in Graphene, Phys. Rev. Lett. 102, 206410 (2009).

[59] J. C. W. Song, M. Y. Reizer, and L. S. Levitov, DisorderAssisted Electron-Phonon Scattering and Cooling Pathways in Graphene, Phys. Rev. Lett. 109, 106602 (2012).

[60] M. W. Graham, S. F. Shi, D. C. Ralph, J. Park, and P. L. McEuen, Photocurrent measurements of supercollision cooling in graphene, Nat. Phys. 9, 103 (2013).

[61] A. C. Betz, S. H. Jhang, E. Pallecchi, R. Ferreira, G. Fève, J.-M. Berroir, and B. Plaçais, Supercollision cooling in undoped graphene, Nat. Phys. 9, 109 (2012).

[62] T. Low, V. Perebeinos, R. Kim, M. Freitag, and P. Avouris, Cooling of photoexcited carriers in graphene by internal and substrate phonons, Phys. Rev. B 86, 045413 (2012).

[63] A. Bostwick, T. Ohta, T. Seyller, K. Horn, and E. Rotenberg, Quasiparticle dynamics in graphene, Nat. Phys. 3, 36 (2007).

[64] F. Rana, J. H. Strait, H. Wang, and C. Manolatou, Ultrafast carrier recombination and generation rates for plasmon emission and absorption in graphene, Phys. Rev. B 84, 045437 (2011).

[65] J. M. Hamm, A. F. Page, J. Bravo-Abad, F. J. Garcia-Vidal, and O. Hess, Nonequilibrium plasmon emission drives ultrafast carrier relaxation dynamics in photoexcited graphene, Phys. Rev. B 93, 041408(R) (2016).

[66] R. K. Willardson and A. C. Beer, A Treatise: Transport Phenomena, Semiconductors and Semimetals (Academic, Orlando, 1972), Vol. 10.

[67] M. Lundstrom, Fundamentals of Carrier Transport, 2nd ed. (Cambridge University Press, Cambridge, 2009).

[68] Y.-W. Tan, Y. Zhang, K. Bolotin, Y. Zhao, S. Adam, E. H. Hwang, S. Das Sarma, H. L. Stormer, and P. Kim, Measurement of Scattering Rate and Minimum Conductivity in Graphene, Phys. Rev. Lett. 99, 246803 (2007).

[69] E. H. Hwang, S. Adam, and S. Das Sarma, Carrier Transport in Two-Dimensional Graphene Layers, Phys. Rev. Lett. 98, 186806 (2007).

[70] S. V. Morozov, K. S. Novoselov, M. I. Katsnelson, F. Schedin, D. C. Elias, J. A. Jaszczak, and A. K. Geim, Giant Intrinsic Carrier Mobilities in Graphene and Its Bilayer, Phys. Rev. Lett. 100, 016602 (2008).

[71] C. R. Dean, A. F. Young, I. Meric, C. Lee, L. Wang, S. Sorgenfrei, K. Watanabe, T. Taniguchi, P. Kim, K. L. Shepard, and J. Hone, Boron nitride substrates for high-quality graphene electronics, Nat. Nanotechnol. 5, 722 (2010).

[72] V. Perebeinos and P. Avouris, Inelastic scattering and current saturation in graphene, Phys. Rev. B 81, 195442 (2010).

[73] S. Tanabe, Y. Sekine, H. Kageshima, M. Nagase, and H. Hibino, Carrier transport mechanism in graphene on SiC(0001), Phys. Rev. B 84, 115458 (2011).

[74] K. Zou, X. Hong, D. Keefer, and J. Zhu, Deposition of HighQuality $\mathrm{HfO}_{2}$ on Graphene and the Effect of Remote Oxide Phonon Scattering, Phys. Rev. Lett. 105, 126601 (2010).

[75] E. H. Hwang and S. Das Sarma, Acoustic phonon scattering limited carrier mobility in two-dimensional extrinsic graphene, Phys. Rev. B 77, 115449 (2008).

[76] E. V. Castro, H. Ochoa, M. I. Katsnelson, R. V. Gorbachev, D. C. Elias, K. S. Novoselov, A. K. Geim, and F. Guinea,
Limits on Charge Carrier Mobility in Suspended Graphene Due to Flexural Phonons, Phys. Rev. Lett. 105, 266601 (2010).

[77] K. Van Nguyen and Y. C. Chang, Full consideration of acoustic phonon scatterings in two-dimensional Dirac materials, Phys. Chem. Chem. Phys. 22, 3999 (2020).

[78] S. Adam, E. H. Hwang, V. M. Galitski, and S. Das Sarma, A self-consistent theory for graphene transport, Proc. Natl. Acad. Sci. USA 104, 18392 (2007).

[79] T. Ando, Screening effect and impurity scattering in monolayer graphene, J. Phys. Soc. Jpn. 75, 074716 (2006).

[80] J.-H. Chen, C. Jang, S. Adam, M. S. Fuhrer, E. D. Williams, and M. Ishigami, Charged-impurity scattering in graphene, Nat. Phys. 4, 377 (2008).

[81] I.-T. Lin and J.-M. Liu, Terahertz frequency-dependent carrier scattering rate and mobility of monolayer and AA-stacked multilayer graphene, IEEE J. Sel. Top. Quantum Electron. 20, 8400108 (2014).

[82] T. Stauber, N. M. R. Peres, and F. Guinea, Electronic transport in graphene: A semiclassical approach including midgap states, Phys. Rev. B 76, 205423 (2007).

[83] S. Adam, E. H. Hwang, and S. Das Sarma, Scattering mechanisms and Boltzmann transport in graphene, Phys. E (Amsterdam) 40, 1022 (2008)

[84] M. I. Katsnelson and A. K. Geim, Electron scattering on microscopic corrugations in graphene, Philos. Trans. R. Soc., A 366, 195 (2008).

[85] A. Pachoud, M. Jaiswal, P. K. Ang, K. P. Loh, and B. Özyilmaz, Graphene transport at high carrier densities using a polymer electrolyte gate, EPL (Europhys. Lett.) 92, 27001 (2010).

[86] J. Yan and M. S. Fuhrer, Correlated Charged Impurity Scattering in Graphene, Phys. Rev. Lett. 107, 206601 (2011).

[87] A. H. Castro Neto, F. Guinea, N. M. R. Peres, K. S. Novoselov, and A. K. Geim, The electronic properties of graphene, Rev. Mod. Phys. 81, 109 (2009).

[88] E. H. Hwang and S. Das Sarma, Screening-induced temperature-dependent transport in two-dimensional graphene, Phys. Rev. B 79, 165404 (2009).

[89] S. Piscanec, M. Lazzeri, J. Robertson, A. C. Ferrari, and F. Mauri, Optical phonons in carbon nanotubes: Kohn anomalies, Peierls distortions, and dynamic effects, Phys. Rev. B 75, 035427 (2007).

[90] K. I. Bolotin, K. J. Sikes, J. Hone, H. L. Stormer, and P. Kim, Temperature-Dependent Transport in Suspended Graphene, Phys. Rev. Lett. 101, 096802 (2008).

[91] J.-h. Chen, C. Jang, S. Xiao, M. Ishigami, and M. S. Fuhrer, Intrinsic and extrinsic performance limits of graphene devices on $\mathrm{SiO}_{2}$, Nat. Nanotechnol. 3, 206 (2008).

[92] X. Hong, A. Posadas, K. Zou, C. H. Ahn, and J. Zhu, High-Mobility Few-Layer Graphene Field Effect Transistors Fabricated on Epitaxial Ferroelectric Gate Oxides, Phys. Rev. Lett. 102, 136808 (2009).

[93] D. K. Efetov and P. Kim, Controlling Electron-Phonon Interactions in Graphene at Ultrahigh Carrier Densities, Phys. Rev. Lett. 105, 256805 (2010).

[94] A. A. Kozikov, A. K. Savchenko, B. N. Narozhny, and A. V. Shytov, Electron-electron interactions in the conductivity of graphene, Phys. Rev. B 82, 075424 (2010).

[95] E. Mariani and F. von Oppen, Temperature-dependent resistivity of suspended graphene, Phys. Rev. B 82, 195403 (2010). 
[96] H. Min, E. H. Hwang, and S. Das Sarma, Chirality-dependent phonon-limited resistivity in multiple layers of graphene, Phys. Rev. B 83, 161404(R) (2011).

[97] K. Kaasbjerg, K. S. Thygesen, and K. W. Jacobsen, Unraveling the acoustic electron-phonon interaction in graphene, Phys. Rev. B 85, 165440 (2012).

[98] H. Ochoa, E. V. Castro, M. I. Katsnelson, and F. Guinea, Scattering by flexural phonons in suspended graphene under back gate induced strain, Phys. E (Amsterdam) 44, 963 (2012).

[99] T. Sohier, M. Calandra, C. H. Park, N. Bonini, N. Marzari, and F. Mauri, Phonon-limited resistivity of graphene by firstprinciples calculations: Electron-phonon interactions, straininduced gauge field, and Boltzmann equation, Phys. Rev. B 90, 125414 (2014).

[100] K. M. Borysenko, J. T. Mullen, E. A. Barry, S. Paul, Y. G. Semenov, J. M. Zavada, M. B. Nardelli, and K. W. Kim, First-principles analysis of electron-phonon interactions in graphene, Phys. Rev. B 81, 121412(R) (2010).

[101] C. H. Park, N. Bonini, T. Sohier, G. Samsonidze, B. Kozinsky, M. Calandra, F. Mauri, and N. Marzari, Electron-phonon interactions and the intrinsic electrical resistivity of graphene, Nano Lett. 14, 1113 (2014).

[102] T. Someya, H. Fukidome, H. Watanabe, T. Yamamoto, M. Okada, H. Suzuki, Y. Ogawa, T. Iimori, N. Ishii, T. Kanai, K. Tashima, B. Feng, S. Yamamoto, J. Itatani, F. Komori, K. Okazaki, S. Shin, and I. Matsuda, Suppression of supercollision carrier cooling in high mobility graphene on $\mathrm{SiC}(000 \overline{1})$, Phys. Rev. B 95, 165303 (2017).

[103] F. Rana, P. A. George, J. H. Strait, J. Dawlaty, S. Shivaraman, M. Chandrashekhar, and M. G. Spencer, Carrier recombination and generation rates for intravalley and intervalley phonon scattering in graphene, Phys. Rev. B 79, 115447 (2009).

[104] H. Wang, J. H. Strait, P. A. George, S. Shivaraman, V. B. Shields, M. Chandrashekhar, J. Hwang, F. Rana, M. G. Spencer, C. S. Ruiz-Vargas, and J. Park, Ultrafast relaxation dynamics of hot optical phonons in graphene, Appl. Phys. Lett. 96, 081917 (2010).

[105] N. Bonini, M. Lazzeri, N. Marzari, and F. Mauri, Phonon Anharmonicities in Graphite and Graphene, Phys. Rev. Lett. 99, 176802 (2007).

[106] E. V. Fedulova, M. M. Nazarov, A. A. Angeluts, M. S. Kitai, V. I. Sokolov, and A. P. Shkurinov, Studying of dielectric properties of polymers in the terahertz frequency range, Proc. SPIE 8337, 83370I (2012).

[107] X. Zhang, J. Qiu, X. Li, J. Zhao, and L. Liu, Complex refractive indices measurements of polymers in visible and near-infrared bands, Appl. Opt. 59, 2337 (2020).

[108] B. T. Hasan, Z. Sun, F. Wang, F. Bonaccorso, P. H. Tan, A. G. Rozhin, and A. C. Ferrari, Nanotube-polymer composites for ultrafast photonics, Adv. Mater. (Weinheim) 21, 3874 (2009).

[109] Q. Bao, H. Zhang, Y. Wang, Z. Ni, and Y. Yan, Atomic-layer graphene as a saturable absorber for ultrafast pulsed lasers, Adv. Funct. Mater. 19, 3077 (2009).

[110] G. Xing, H. Guo, X. Zhang, T. C. Sum, C. Hon, and A. Huan, The physics of ultrafast saturable absorption in graphene, Opt. Express 18, 4564 (2010).

[111] A. Marini, J. D. Cox, and F. J. García de Abajo, Theory of graphene saturable absorption, Phys. Rev. B 95, 125408 (2017).
[112] S. Fratini and F. Guinea, Substrate-limited electron dynamics in graphene, Phys. Rev. B 77, 195415 (2008).

[113] X. Li, E. A. Barry, J. M. Zavada, M. Buongiorno Nardelli, and K. W. Kim, Surface polar phonon dominated electron transport in graphene, Appl. Phys. Lett. 97, 232105 (2010).

[114] A. Konar, T. Fang, and D. Jena, Effect of high- $\kappa$ gate dielectrics on charge transport in graphene-based field effect transistors, Phys. Rev. B 82, 115452 (2010).

[115] E. H. Hwang and S. Das Sarma, Surface polar optical phonon interaction induced many-body effects and hot-electron relaxation in graphene, Phys. Rev. B 87, 115432 (2013).

[116] K. J. Tielrooij, N. C. Hesp, A. Principi, M. B. Lundeberg, E. A. Pogna, L. Banszerus, Z. Mics, M. Massicotte, P. Schmidt, D. Davydovskaya, D. G. Purdie, I. Goykhman, G. Soavi, A. Lombardo, K. Watanabe, T. Taniguchi, M. Bonn, D. Turchinovich, C. Stampfer, A. C. Ferrari et al., Out-of-plane heat transfer in van der Waals stacks through electron-hyperbolic phonon coupling, Nat. Nanotechnol. 13, 41 (2018).

[117] M. Müller, M. Bräuninger, and B. Trauzettel, Temperature Dependence of the Conductivity of Ballistic Graphene, Phys. Rev. Lett. 103, 196801 (2009).

[118] V. P. Gusynin, S. G. Sharapov, and J. P. Carbotte, On the universal ac optical background in graphene, New J. Phys. 11, 095013 (2009).

[119] M. Wagner, Z. Fei, A. S. McLeod, A. S. Rodin, W. Bao, E. G. Iwinski, Z. Zhao, M. Goldflam, M. Liu, G. Dominguez, M. Thiemens, M. M. Fogler, A. H. Castro Neto, C. N. Lau, S. Amarie, F. Keilmann, and D. N. Basov, Ultrafast and nanoscale plasmonic phenomena in exfoliated graphene revealed by infrared pump-probe nanoscopy, Nano Lett. 14, 894 (2014).

[120] M. Yamashita, H. Takahashi, T. Ouchi, and C. Otani, Ultrabroadband terahertz time-domain ellipsometric spectroscopy utilizing $\mathrm{GaP}$ and GaSe emitters and an epitaxial layer transferred photoconductive detector, Appl. Phys. Lett. 104, 051103 (2014).

[121] D. C. Elias, R. V. Gorbachev, A. S. Mayorov, S. V. Morozov, A. A. Zhukov, P. Blake, L. A. Ponomarenko, I. V. Grigorieva, K. S. Novoselov, F. Guinea, and A. K. Geim, Dirac cones reshaped by interaction effects in suspended graphene, Nat. Phys. 7, 701 (2011).

[122] X. Xie, J. Dai, and X. C. Zhang, Coherent Control of THz Wave Generation in Ambient Air, Phys. Rev. Lett. 96, 075005 (2006).

[123] J. Dai, X. Xie, and X. C. Zhang, Detection of Broadband Terahertz Waves with a Laser-Induced Plasma in Gases, Phys. Rev. Lett. 97, 103903 (2006).

[124] I. Hyung, B. Hwang, W. Lee, S. Bae, B. Hee, H. Yeong, H. Ahn, and D.-i. Y. Fabian, Efficient mode-locking of sub-70-fs Ti:sapphire laser by graphene saturable absorber, Appl. Phys. Express 5, 032701 (2012).

[125] K. Kang, D. Abdula, D. G. Cahill, and M. Shim, Lifetimes of optical phonons in graphene and graphite by time-resolved incoherent anti-Stokes Raman scattering, Phys. Rev. B 81, 165405 (2010).

[126] B. Gao, G. Hartland, T. Fang, M. Kelly, D. Jena, H. Xing, and L. Huang, Studies of intrinsic hot phonon dynamics in 
suspended graphene by transient absorption microscopy, Nano Lett. 11, 3184 (2011).

[127] G. Froehlicher and S. Berciaud, Raman spectroscopy of electrochemically gated graphene transistors: Geometrical capacitance, electron-phonon, electron-electron, and electrondefect scattering, Phys. Rev. B 91, 205413 (2015).

[128] A. Das, S. Pisana, B. Chakraborty, S. Piscanec, S. K. Saha, U. V. Waghmare, K. S. Novoselov, H. R. Krishnamurthy, A. K. Geim, A. C. Ferrari, and A. K. Sood, Monitoring dopants by
Raman scattering in an electrochemically top-gated graphene transistor, Nat. Nanotechnol. 3, 210 (2008).

[129] A. Das, B. Chakraborty, S. Piscanec, S. Pisana, A. K. Sood, and A. C. Ferrari, Phonon renormalization in doped bilayer graphene, Phys. Rev. B 79, 155417 (2009).

[130] Y. V. Bludov, N. M. Peres, and M. I. Vasilevskiy, Unusual reflection of electromagnetic radiation from a stack of graphene layers at oblique incidence, J. Opt. (Bristol) 15, 114004 (2013). 